\title{
HAMILTONIAN AND SYMPLECTIC SYMMETRIES: AN INTRODUCTION
}

\author{
ÁlVARO PELAYO \\ In memory of Professor J.J. Duistermaat (1942-2010)
}

\begin{abstract}
Classical mechanical systems are modeled by a symplectic manifold $(M, \omega)$, and their symmetries are encoded in the action of a Lie group $G$ on $M$ by diffeomorphisms which preserve $\omega$. These actions, which are called symplectic, have been studied in the past forty years, following the works of Atiyah, Delzant, Duistermaat, Guillemin, Heckman, Kostant, Souriau, and Sternberg in the 1970s and 1980s on symplectic actions of compact Abelian Lie groups that are, in addition, of Hamiltonian type, i.e., they also satisfy Hamilton's equations. Since then a number of connections with combinatorics, finitedimensional integrable Hamiltonian systems, more general symplectic actions, and topology have flourished. In this paper we review classical and recent results on Hamiltonian and non-Hamiltonian symplectic group actions roughly starting from the results of these authors. This paper also serves as a quick introduction to the basics of symplectic geometry.
\end{abstract}

\section{INTRODUCTION}

Symplectic geometry is concerned with the study of a notion of signed area, rather than length, distance, or volume. It can be, as we will see, less intuitive than Euclidean or metric geometry and it is taking mathematicians many years to understand its intricacies (which is work in progress).

The word "symplectic" goes back to the 1946 book 164 by Hermann Weyl (1885-1955) on classical groups. It derives from a Greek word meaning "complex". Since the word complex already had a precise meaning in mathematics, and was already used at the time of Weyl, he took the Latin root of complex (which means "plaited together") and replaced it by the Greek root "symplectic".

The origins of symplectic geometry are in classical mechanics, where the phase space of a mechanical system is modeled by a symplectic manifold $(M, \omega)$, that is, a smooth manifold $M$ endowed with a nondegenerate closed 2-form $\omega \in \Omega^{2}(M)$, called a symplectic form. At each point $x \in M, \omega_{x}: \mathrm{T}_{x} M \times \mathrm{T}_{x} M \rightarrow \mathbb{R}$ is a skewsymmetric bilinear map, and given $u, v \in \mathrm{T}_{x} M$ the real number $\omega_{x}(u, v)$ is called the symplectic area spanned by $u$ and $v$. Intuitively, $\omega$ gives a way to measure area along two-dimensional sections of $M$. The most typical example of a symplectic manifold is a cotangent bundle, which comes endowed with a canonical symplectic form.

Received by the editors October 14, 2016.

2010 Mathematics Subject Classification. Primary 53D20, 53D35, 57R17, 37J35, 57M60, $58 \mathrm{D} 27,57 \mathrm{~S} 25$.

The author is supported by NSF CAREER Grant DMS-1518420. 
Initially it was the study of mechanical systems which motivated many of the developments in symplectic geometry. Joseph-Louis Lagrange (1736-1813) gave the first example of a symplectic manifold in 1808 in his study of the motion of the planets under the influence of their mutual gravitational interaction [102,103]. An explicit description of Lagrange's construction and his derivation of what are known today as Hamilton's equations is given by Weinstein in [160, Section 2]. The origins of the current viewpoint in symplectic geometry may be traced back to Carl Gustav Jacob Jacobi (1804-1851) and then William Rowan Hamilton's (1805-1865) deep formulation of Lagrangian mechanics around 1835. Hamilton was expanding on and reformulating ideas of Galileo Galilei (1564-1642), Christiaan Huygens (1629-1695), Leonhard Euler (1707-1883), Lagrange, and Isaac Newton (1642-1727) about the structure and behavior of orbits of planetary systems.

At the time of Newton and Huygens the point of view in classical mechanics was geometric. Later Lagrange, Jacobi, and Hamilton approached the subject from an analytic viewpoint. Through their influence the more geometric viewpoint fell out of fashion. Further historical details and references are given by Weinstein in 160. Several treatments of mechanical systems in the 1960s and 1970s, notably including [1,2,7, 9, 151, had an influence in the development of symplectic geometry.

The modern viewpoint in symplectic geometry starts with the important contributions of a number of authors in the early 1970s (some slightly before or slightly after), including the works of Ralph Abraham, Vladimir Arnold, Johannes J. Duistermaat, Victor Guillemin, Bertram Kostant, Paulette Libermann, George Mackey, Jerrold Marsden, Clark Robinson, Jean-Marie Souriau, Shlomo Sternberg, and Alan Weinstein. Even at these early stages, many other authors contributed to aspects of the subject so the list is extensive and we do not make an attempt to cover it.

Symplectic geometry went through a series of developments in the period from 1970 to 1985 where connections with other areas flourished, including: (i) microlocal and semiclassical analysis, as in the works of Duistermaat and Hörmander [35, 37, where Duistermaat played a leading role in establishing relations between the microlocal and symplectic communities in particular through his article on oscillatory integrals and Lagrange immersions [35; (ii) completely integrable systems, of which Duistermaat's article on global action-angle coordinates [36] may be considered to mark the beginning of the global theory; (iii) Poisson geometry, as in Weinstein's foundational article [161]; (iv) Lie theory and Kostant and Souriau's geometric quantization [98, 149] (in the early 1960s the quantum viewpoint had already reached significant relevance in mathematics, see Mackey's mathematical foundations of quantum mechanics [108]), on which the works by Segal [147] and Kirillov [94 had an influence; and (v) symplectic and Hamiltonian group actions, as pioneered by Atiyah [10, Duistermaat and Heckman [37, Guillemin and Sternberg [75], Kostant [97, and Souriau [150].

An influential precursor in the study of global aspects in symplectic geometry, the study of which is often referred to as symplectic topology, is Arnold's Conjecture [9, Appendix 9] (a particular case appeared in [8]; see Zehnder's article [167] for an expository account). Arnold's Conjecture is a higher-dimensional analogue of the classical fixed point theorem of Henri Poincaré (1854-1912) and George Birkhoff (1884-1944) which says that any area-preserving twist of a closed annulus has at least two geometrically distinct fixed points. This fixed point result can be traced to the work of Poincaré in celestial mechanics [141, where he showed that the 
study of the dynamics of certain cases of the restricted Three-Body Problem may be reduced to investigating area-preserving maps. This work led Poincaré to the theorem, which he stated in [142] in 1912, and proved in several cases. The complete proof was given by Birkhoff [19] in 1913.

Arnold realized that the higher-dimensional version of the result of Poincaré and Birkhoff should concern symplectic maps, that is, maps preserving a symplectic form, rather than volume-preserving maps, and formulated his conjecture. Arnold's Conjecture has been responsible for many developments in symplectic geometry (as well as in subjects such as Hamiltonian dynamics).

In 1985 Gromov [66] introduced pseudo-holomorphic curve techniques into symplectic geometry and constructed the first so-called symplectic capacity, a notion of monotonic symplectic invariant later pioneered by Ekeland and Hofer [44, 80, 81] and developed by Hofer and his collaborators, as well as many others, from the angle of dynamical systems and Hamiltonian dynamics.

There have been many major developments since the early 1980s and on many different fronts of symplectic geometry and topology; covering them (even very superficially) would be beyond the scope of this paper. In this article we study only on the topic of symplectic and Hamiltonian group actions (item (v) above) starting roughly with the work of Atiyah and of Guillemin and Sternberg.

The phase space of a mechanical system is modeled by a symplectic manifold, and its symmetries are described by symplectic group actions. The study of these actions fits into a large body of work by the name of equivariant symplectic geometry, which includes tools of current interest also in algebraic geometry, such as equivariant cohomology, on which we will briefly touch.

Mathematically speaking, equivariant symplectic geometry is concerned with the study of smooth actions of Lie groups $G$ on symplectic manifolds $M$ by means of diffeomorphisms $\varphi: M \rightarrow M$ that pullback the symplectic form $\omega$ to itself: $\varphi^{*} \omega=\omega$. A map $\varphi$ satisfying this condition is called a symplectomorphism, following Souriau, or a canonical transformation. Actions satisfying this natural condition are called symplectic. As a first example, let $S^{1}$ be the group of unit complex numbers, let $S^{2}$ be the unit sphere, and endow $M=S^{2} \times\left(S^{1}\right)^{2}$ with the product form of any area forms on $S^{2}$ and $\left(S^{1}\right)^{2}$. Then the action of $G=\left(S^{1}\right)^{2}$ by multiplication on the right factor is symplectic.

In this paper we treat primarily the case when $G$ is a compact, connected, Abelian Lie group; that is, a torus $T \simeq\left(S^{1}\right)^{k}$. Let $\mathfrak{t}$ be the Lie algebra of $T$, and let $\mathfrak{t}^{*}$ be its dual Lie algebra. We think of $\mathfrak{t}$ as the tangent space at $1 \in T$. Equivalently, a $T$-action is symplectic if $\mathrm{L}_{X_{M}} \omega=0$ for every $X \in \mathfrak{t}$, where $\mathrm{L}$ is the Lie derivative and $X_{M}$ is the vector field generated by the $T$-action from $X$ by the exponential map. In view of the homotopy formula for the Lie derivative, this says

$$
\mathrm{d}\left(\omega\left(X_{M}, \cdot\right)\right)=0, \quad \forall X \in \mathfrak{t} .
$$

A fundamental subclass of symplectic actions admits a so-called momentum map $\mu: M \rightarrow \mathfrak{t}^{*}$, which is a $\mathfrak{t}^{*}$-valued smooth function on $M$ which encodes information about $M$ itself, the symplectic form, and the $T$-action, and it is characterized by the condition

$$
-\mathrm{d}\langle\mu, X\rangle=\omega\left(X_{M}, \cdot\right), \quad \forall X \in \mathfrak{t} .
$$

Such special symplectic actions are called Hamiltonian (the momentum map was introduced for any Lie group action by Kostant [97] and Souriau [149]). An example 
is given by $S^{2}$ and $T=S^{1}$ acting by rotations about the $z$-axis. In this case $\mathfrak{t}^{*} \simeq \mathbb{R}$ and, in spherical coordinates, $\mu:(\theta, h) \mapsto h$.

The fundamental fact here is that the right-hand side of equation (1.2) is a closed 1-form by (1.1) and being Hamiltonian may be rephrased as the requirement that this form be exact. This is an extremely stringent condition-for instance, by (1.2) it forces the action to have fixed points on a compact manifold. The obstruction for a symplectic action to be Hamiltonian lies in the cohomology group $\mathrm{H}^{1}(M ; \mathbb{R})$, so symplectic actions on simply connected manifolds are Hamiltonian.

The advantage of having the existence of a momentum map $\mu: M \rightarrow \mathfrak{t}^{*}$ for a symplectic action has led to a rich general theory, part of which is described in this article. One can often find out information about $(M, \omega)$ and the $T$-action through the study of $\mu$. For instance, if $M$ is compact and connected, the $T$-action is effective, and $\operatorname{dim} M=2 \operatorname{dim} T$, Delzant proved [33] that the image $\mu(M) \subset \mathfrak{t}^{*}$ characterizes $(M, \omega)$ and the $T$-action up to symplectic and $T$-equivariant transformations.

Hamiltonian actions have been extensively studied since the 1970s following the seminal works of Atiyah [10, Delzant [33, Duistermaat and Heckman 37], Guillemin and Sternberg [75], Kostant [97, and Souriau [150, and they have motivated the study of more general symplectic actions. Many symplectic actions of interest in complex algebraic geometry and Kähler geometry are symplectic but not Hamiltonian; one such case is the action of the 2-torus on the Kodaira variety, which appears in Kodaira's description [96. Theorem 19] of the compact complex analytic surfaces which have a holomorphic $(2,0)$-form that is nowhere vanishing, described later (Example 5.10).

Other symplectic actions which do not admit a momentum map include examples of interest in differential geometry (e.g., multiplicty free spaces), and topology (e.g., nilmanifolds over nilpotent Lie groups). Recently in [154, Susan Tolman has constructed an example of a symplectic non-Hamiltonian $S^{1}$-action with some, but only finitely many, fixed points on a compact manifold (Theorem 3.14).

This paper is not a survey (which would require, due to the volume of works, a longer paper) but rather a brief introduction to symmetries in symplectic geometry. Approximately the first half of the paper concerns the period from 1970 to 2002, where the emphasis is on Hamiltonian actions, its applications, and its implications, including the interactions with integrable systems. The second half of the paper concerns symplectic actions which are not necessarily Hamiltonian, with a focus on developments that took place in the approximate period from 2002 to 2015. Of course this separation is somewhat artificial, because Hamiltonian actions play a fundamental role in the study of general symplectic actions. We will cover a few representative proofs; most proofs about general symplectic actions use in an essential way the Hamiltonian theory but also include other ingredients. Since there is no momentum map $\mu$ in general, Morse theory for $\mu$ and Duistermaat-Heckman theory, often used in the Hamiltonian case, must be replaced by other techniques.

Outline of topics. Section 2 gives an introduction to symplectic geometry. Sections 3 and 4 introduce the basics of symplectic and Hamiltonian Lie group actions. Section 5 contains examples of Hamiltonian and symplectic non-Hamiltonian torus actions. Section [6 includes classification results on symplectic Hamiltonian Lie group actions. In most cases, the Lie group is compact connected and Abelian, but a certain case of noncompact groups, which is pertinent to completely integrable 
systems, is also included. The material in Sections 7 and 8 focuses on developments on symplectic non-Hamiltonian actions in the past 15 years, with an emphasis on classification results.

\section{SympleCtiC MANIFOLDS}

Symplectic geometry is concerned with the study a notion of signed area, and it displays a degree of flexibility and rigidity at the same time which makes it a rich subject, the study of which is of interest well beyond its original connection to classical mechanics.

2.1. Basic properties. For the basics of symplectic geometry we recommend the books $[22,83,116]$. This section gives an overview of the basic notions of the subject and presents the background needed for the following sections. Unless otherwise specified, manifolds are $\mathrm{C}^{\infty}$-smooth and have no boundary.

Definition 2.1. A symplectic manifold is a pair $(M, \omega)$ consisting of a smooth manifold $M$ and a smooth 2 -form $\omega$ on $M$ which is closed (i.e., d $\omega=0$ ) and nondegenerate (i.e., for each $x \in M$ it holds that if $u \in \mathrm{T}_{x} M$ is such that $\omega_{x}(u, v)=$ 0 for all $v \in \mathrm{T}_{x} M$ at $x$, then necessarily $u=0$ ).

The form $\omega$ is called a symplectic form. At each $x \in M, \omega_{x}: \mathrm{T}_{x} M \times \mathrm{T}_{x} M \rightarrow \mathbb{R}$ is a skew-symmetric bilinear map. If $M=W$ is a vector space and $\omega: W \times W \rightarrow \mathbb{R}$ is a skew-symmetric bilinear map, the pair $(W, \omega)$ is a symplectic manifold (often called a symplectic vector space).

Example 2.2. In dimension 2, a symplectic form is an area form. Accordingly, a surface with an area form is a symplectic manifold. A typical noncompact example is the Euclidean space $\mathbb{R}^{2 n}$ with coordinates $\left(x_{1}, y_{1}, \ldots, x_{n}, y_{n}\right)$ and symplectic form $\sum_{i=1}^{n} \mathrm{~d} x_{i} \wedge \mathrm{d} y_{i}$. Any open subset $U$ of $\mathbb{R}^{2 n}$ endowed with the symplectic form given by this same formula is also a symplectic manifold.

Proposition 2.3. Let $X$ be a smooth $n$-dimensional manifold and let $\left(V, x_{1}, \ldots, x_{n}\right)$ be a smooth chart for $X$. To this chart we can associate a cotangent bundle chart $\left(\mathrm{T}^{*} V, x_{1}, \ldots, x_{n}, \xi_{1}, \ldots, \xi_{n}\right)$ on which we can define, in coordinates, the smooth 2 form $\sum_{i=1}^{n} \mathrm{~d} x_{i} \wedge \mathrm{d} \xi_{i}$. Then the expression for the 2-form is coordinate-independent, that is, it defines a canonical smooth 2 -form $\omega_{\mathrm{T}^{*} X}$ on the cotangent bundle $\mathrm{T}^{*} X$, which is, moreover, symplectic and exact.

Proof. It follows from $\sum_{i=1}^{n} \mathrm{~d} x_{i} \wedge \mathrm{d} \xi_{i}=-\mathrm{d} \alpha$ where $\alpha:=\sum_{i=1}^{n} \xi_{i} \mathrm{~d} x_{i}$, since $\alpha$ is intrinsically defined (by a simple calculation using the definition of cotangent bundle chart).

A way to construct symplectic manifolds is by taking products and endowing them with the product form; so $S^{2} \times \mathbb{R}^{2 n}, \mathrm{~T}^{*} X \times \mathrm{T}^{*} Y$ are symplectic manifolds, where $X$ and $Y$ are any manifolds.

There is a geometric interpretation of the closedness of a symplectic form as follows. If $(M, \omega)$ is a symplectic manifold and $S \subset M$ is an oriented surface, with or without boundary, we define the following:

$$
\text { symplectic area of } S:=\int_{S} \omega \in \mathbb{R} \text {. }
$$


Proposition 2.4. Every point in a symplectic manifold $(M, \omega)$ has an open neighborhood $U$ such that if the oriented surface $S$ is contained in $U$, then the symplectic area of $S$ depends only on the boundary $\partial S$ of $S$, in the sense that it does not change when smoothly deforming $S$ inside of $U$ while keeping $\partial S$ fixed under the deformation. If $\omega$ is exact, then $U=M$.

Proof. If $\mathrm{d} \omega=0$, locally near any $x \in M, \omega=\mathrm{d} \sigma$ for some $\sigma \in \Omega^{1}(M)$. So by Stokes' theorem,

and the result follows.

$$
\int_{S} \omega=\int_{S} \mathrm{~d} \sigma=\int_{\partial S} \sigma
$$

By Propositions 2.3 and 2.4 the symplectic area of any oriented surface $S$ in a cotangent bundle $\left(\mathrm{T}^{*} X, \omega_{\mathrm{T}^{*} X}\right)$ depends only on $\partial S$, and if $\partial S=\varnothing$, it is zero. The nondegeneracy of $\omega$ gives:

Proposition 2.5. If $(M, \omega)$ is a symplectic manifold, then the mapping

$$
\mathcal{X} \mapsto \omega(\mathcal{X}, \cdot)
$$

is an isomorphism between the tangent and the cotangent bundles $\mathrm{T} M \rightarrow \mathrm{T}^{*} M$.

In other words, the symplectic form gives a correspondence between one-forms and vector fields.

One can ask some basic questions about symplectic manifolds. For instance, one can wonder:

Question 2.6. Does the three-dimensional sphere $S^{3}$ admit a symplectic form?

The answer is "no", because symplectic manifolds are even-dimensional; for otherwise the nondegeneracy condition is violated, which follows from linear algebra. Similarly:

Question 2.7. Is there a nonorientable smooth manifold that admits a symplectic form?

The answer is "no", because the nondegeneracy of $\omega$ implies that

$$
\omega^{n}=\omega \wedge \cdots\left(n_{\text {times }}\right) \cdots \wedge \omega
$$

is a volume form giving an orientation to $M$, where $2 n=\operatorname{dim} M$. To summarize:

Proposition 2.8. Symplectic manifolds are even dimensional and orientable.

Question 2.9. Does the four-dimensional sphere $S^{4}$ admit a symplectic form?

Proposition 2.10. If $(M, \omega)$ is a compact symplectic manifold of dimension $2 n$, its even-dimensional cohomology groups $\mathrm{H}^{2 k}(M ; \mathbb{R}) \neq 0,0 \leqslant k \leqslant n$, are nontrivial. Proof. By Stokes' theorem, since $\mathrm{d} \omega=0,\left[\omega^{k}\right]$ is nontrivial in $\mathrm{H}^{2 k}(M ; \mathbb{R}), 1 \leqslant k \leqslant$ $n$.

Corollary 2.11. The 2-sphere $S^{2}$ is the only sphere $S^{n}, n \geqslant 1$, which admits a symplectic form.

The question of whether a manifold admits a symplectic form is difficult. For instance, if $N$ is a compact oriented 3-manifold, Friedl and Vidussi [59] and Kutluhan and Taubes [101 have studied when a compact 4-manifold of the form $S^{1} \times N$ admits a symplectic form, which turns out to imply that $N$ must fiber over the circle $S^{1}$. 
2.2. Symplectomorphisms. The natural maps between symplectic manifolds are the diffeomorphisms which preserve the symplectic structure, they are called canonical transformations, symplectic diffeomorphisms, or, following Souriau [150, symplectomorphisms.

Definition 2.12. A symplectomorphism $\varphi:(M, \omega) \rightarrow\left(M^{\prime}, \omega^{\prime}\right)$ between symplectic manifolds is a diffeomorphism $\varphi: M \rightarrow M^{\prime}$ which is also symplectic, that is, it satisfies $\varphi^{*} \omega^{\prime}=\omega$. In this case we say that $(M, \omega)$ and $\left(M^{\prime}, \omega^{\prime}\right)$ are symplectomorphic.

Recall that $\varphi^{*} \omega^{\prime}=\omega$ means $\omega_{\varphi(x)}^{\prime}\left(\mathrm{d}_{x} \varphi(u), \mathrm{d}_{x} \varphi(v)\right)=\omega_{x}(u, v)$ for every $x \in M$ and $u, v \in \mathrm{T}_{x} M$.

Remark 2.13. Roughly speaking, one may view symplectomorphisms as diffeomorphisms preserving the area enclosed by loops or, rather, the sum of the areas enclosed by their projections onto a collection of two-dimensional planes. For instance, if $(M, \omega)=\left(\mathbb{R}^{6}, \mathrm{~d} x_{1} \wedge \mathrm{d} y_{1}+\mathrm{d} x_{2} \wedge \mathrm{d} y_{2}+\mathrm{d} x_{3} \wedge \mathrm{d} y_{3}\right)$, with coordinates $\left(x_{1}, y_{1}, x_{2}, y_{2}, x_{3}, y_{3}\right)$, then you would want to preserve the area (counted by $\omega$ with sign depending on the orientation of the region inside) of the projection of any loop in $\mathbb{R}^{6}$ onto the $\left(x_{1}, y_{1}\right),\left(x_{2}, y_{2}\right)$, and $\left(x_{3}, y_{3}\right)$ planes. We learned how to think about symplectomorphisms in this way from Helmut Hofer. An equivalent description of symplectomorphisms was given for $\left(\mathbb{R}^{2 n}, \sum_{i=1}^{n} \mathrm{~d} x_{i} \wedge \mathrm{d} y_{i}\right)$ by Arnold [7, pages 238, 239, Section E on "Canonical Transformations"].

The symplectic volume, or Liouville volume, of a symplectic manifold of dimension $2 n$ is

$$
\operatorname{vol}(M, \omega):=\frac{1}{n !} \int_{M} \omega^{n} .
$$

Of course, since symplectomorphisms preserve $\omega$, they preserve the symplectic volume, but the converse is in general false (we discuss this in Section 2.6).

Since the late nineteenth century it has been known that symplectic manifolds have no local invariants except for dimension. This is a result due to Jean-Gaston Darboux (1842-1917).

Theorem 2.14 (Darboux [32]). Let $(M, \omega)$ be a symplectic $2 n$-dimensional manifold. Near each point $x_{0} \in M$, there are coordinates $\left(x_{1}, y_{1}, \ldots, x_{n}, y_{n}\right)$ in which $\omega=\sum_{i=1}^{n} \mathrm{~d} x_{i} \wedge \mathrm{d} y_{i}$. That is, any two symplectic manifolds $(M, \omega)$ and $\left(M^{\prime}, \omega^{\prime}\right)$ of the same dimension are locally symplectomorphic near any choice of points $x_{0} \in M$ and $x_{0}^{\prime} \in M^{\prime}$.

Theorem 2.14 gives an essential difference between symplectic and Riemannian geometry, where the curvature is a local invariant.

Remark 2.15. Despite Darboux's theorem, there are local aspects in symplectic geometry which have only been understood recently. In these cases one is not concerned with the local properties of the symplectic form itself but instead with a geometric object, such as a Lie group action, a vector field, etc. We will see many examples of this later in the paper.

In 1981 Weinstein referred to symplectic geometry [160] as "the more flexible geometry of canonical (in particular, area preserving) transformations instead of the rigid geometry of Euclid; accordingly, the conclusions of the geometrical arguments are often qualitative rather than quantitative." 
Manifestations of rigidity in symplectic geometry were discovered in the early days of modern symplectic geometry by Yakov Eliashberg, Mikhael Gromov, and others.

Theorem 2.16 (Eliashberg and Gromov [45, 46, 67]). The group of symplectomorphisms of a compact symplectic manifold is $\mathrm{C}^{0}$-closed in the group of diffeomorphisms.

The group of symplectomorphisms of a manifold has a rich structure, and basic questions about it remain open; see Leonid Polterovich's book [143. Interest in the behavior of symplectic matrices may be found in the early days of symplectic geometry in important work of Clark Robinson [144, 145]. See also Arnold [6] for work in a related direction.

2.3. Fixed point theorems. Poincaré showed [141] that questions concerning the dynamics of certain cases of the restricted Three-Body Problem may be reduced to questions involving area-preserving maps. He concluded that there is no reasonable way to solve the problem explicitly in the sense of finding formulae for the trajectories. Instead of aiming at finding explicitly the trajectories, when studying dynamical systems the goal becomes to understand the analytical and topological behavior of the trajectories. Of a particular interest are the constant ones, that is, the fixed points. The development of the modern field of dynamical systems was influenced by Poincaré's work in celestial mechanics, which led him to the Poincaré-Birkhoff theorem [19,142] in 1912, a result which assures the existence of fixed points of certain area-preserving maps on an annulus. This result has had a major influence in symplectic geometry. It was proved in full by Birkhoff in 1925 .

We will formulate the result equivalently for the strip $\mathcal{S}:=\mathbb{R} \times[-1,1]$ covering the annulus. A diffeomorphism $F=(Q, P)$ of $\mathcal{S}$ is an area-preserving periodic twist if the following conditions hold: $F$ preserves area; $F$ preserves $\mathbb{R} \times\{ \pm 1\}$ (boundary invariance); $F$ is orientation preserving and $\pm Q(q, \pm 1)> \pm q$ for all $q$ (boundary twisting); and $F(q+1, p)=(1,0)+F(q, p)$ for all $p, q$ (periodicity). Two fixed points $\left(q_{1}, p_{1}\right)$ and $\left(q_{2}, p_{2}\right)$ of $F$ are geometrically distinct if $p_{1} \neq p_{2}$ or $q_{1}-q_{2}$ is not an integer.

Theorem 2.17 (Poincaré and Birkhoff [19,142]). An area-preserving periodic twist $F: \mathcal{S} \rightarrow \mathcal{S}$ has at least two geometrically distinct fixed points.

There are many generalizations of this result; see for instance [58, 132 and the references therein.

Arnold formulated the higher-dimensional analogue, the Arnold Conjecture [9] (see also [14,82,83, 167]), which we discuss next.

Let $(M, \omega)$ be a compact symplectic manifold, let $H: \mathbb{R} \times M \rightarrow \mathbb{R}$ be a smooth function, and set $H_{t}(x):=H(t, x)$ for every $t \in \mathbb{R}$. Using $\omega$ and $H_{t}$, we define a vector field $\mathcal{X}_{t}$, the time-dependent exact Hamiltonian vector field associated with $H$, by $\omega\left(\mathcal{X}_{t}, \cdot\right)=-\mathrm{d} H_{t}$. Then $\mathrm{d} \varphi_{t} / \mathrm{d} t=\mathcal{X}_{t}\left(\varphi_{t}\right), \varphi_{0}=$ Identity, defines a family $\left\{\varphi_{t}\right\}_{t \in \mathbb{R}}$ of symplectomorphisms of $(M, \omega)$.

A map $\varphi$ on $M$ is Hamiltonian if it belongs to the flow $\varphi_{t}$ of any time-dependent exact Hamiltonian vector field on $M$. The Arnold Conjecture states that a Hamiltonian map on a compact symplectic manifold has at least as many fixed points as a function on it has critical points; see Zehnder [167.

Weinstein 162 observed that the conjecture holds on compact manifolds when the Hamiltonian map belongs to the flow of a sufficiently small Hamiltonian vector 
field. The first breakthrough on the conjecture was by Conley and Zehnder [29], who proved it for the $2 n$-torus (a proof using generating functions was later given by Chaperon [25]). The second breakthrough was by Floer [53 56].

2.4. Stability properties. The term stability concerns the problem of when two volume forms (or two symplectic forms) can be intertwined by a diffeomorphism. Of particular interest is to understand when the diffeomorphism type of a smooth family of volume forms (or symplectic forms) remains constant, or stable. We start by discussing the simplest case: When are two volume forms on the same manifold diffeomorphic? In 1965 Jürgen Moser proved the following.

Theorem 2.18 (Moser [118]). If $\omega$ and $\tau$ are volume forms on a compact connected oriented smooth manifold with

$$
\int_{M} \omega=\int_{M} \tau
$$

then there exists a diffeomorphism $\varphi: M \rightarrow M$ such that $\varphi^{*} \tau=\omega$.

Remark 2.19. It follows that the total symplectic area of $S^{2}$ given by (2.1) completely determines the symplectic form on $S^{2}$ (and $S^{2}$ is the only symplectic sphere according to Corollary 2.11).

This result is extended to fiber bundles in [92]. If the manifold is noncompact we have the following.

Theorem 2.20 (Greene and Shiohama 68]). Let $M$ be a noncompact connected oriented smooth manifold, and let $\omega$ and $\tau$ be volume forms on $M$ such that

$$
\int_{M} \omega=\int_{M} \tau \leqslant \infty
$$

Suppose that for every end $\epsilon$ of $M, \epsilon$ has finite volume with respect to $\omega$ if and only if $\epsilon$ has finite volume with respect to $\tau$. Then there is a diffeomorphism $\varphi: M \rightarrow M$ such that $\varphi^{*} \tau=\omega$.

Without the assumption on the ends, the conclusion may not hold [68, page 406].

This result has been extended to fiber bundles with noncompact fibers in 134. The simplest version of this statement is for trivial bundles. In this case instead of considering two volume forms, we consider two smooth families of volume forms $\omega_{t}, \tau_{t}$, indexed by a compact manifold $B$. For each $t \in B$, Theorem 2.20 produces a volume-preserving diffeomorphism $\varphi_{t}$ but gives no information on how $\varphi_{t}$ changes with $t$. In 134 it is shown that the $\varphi_{t}$ may be chosen to vary smoothly with $t$.

Moser also proved in 1965 an important stability result for symplectic forms:

Theorem 2.21 (Moser [118]). If $\left\{\omega_{t}\right\}_{t \in[0,1]}$ is a smooth family of cohomologous symplectic forms on a compact connected smooth manifold $M$, then there exists a smooth family $\left\{\varphi_{t}\right\}_{t \in[0,1]}$ of diffeomorphisms of $M$ such that $\varphi_{t}^{*} \omega_{t}=\omega_{0}$, and $\varphi_{0}$ is the identity on $M$.

Moser introduced a method, known as Moser's method (or Moser's trick), to prove this stability result; we discuss it next. Let $\mathrm{L}_{\mathcal{X}}$ be the Lie derivative with respect to a vector field $\mathcal{X}$ on $M$, and let $\mathrm{i}_{\mathcal{X}} \omega$ be the inner product of $\omega$ with $\mathcal{X}$, obtained by inserting $\mathcal{X}$ in the first slot of $\omega$. Recall the homotopy identity

$$
\mathrm{L}_{\mathcal{X}}=\mathrm{i}_{\mathcal{X}} \circ \mathrm{d}+\mathrm{d} \circ \mathrm{i}_{\mathcal{X}}
$$


Moser's far-reaching idea was to differentiate the equation $\varphi_{t}^{*} \omega_{t}=\omega_{0}$ to get

$$
\varphi_{t}^{*}\left(\frac{\mathrm{d}}{\mathrm{d} t} \omega_{t}+\mathrm{L}_{\mathcal{X}_{t}} \omega_{t}\right)=0
$$

where $\mathcal{X}_{t}$ is the time-dependent vector field generating $\left\{\varphi_{t}\right\}_{t \in[0,1]}$. By (2.3), $\mathrm{L}_{\mathcal{X}_{t}} \omega_{t}=$ $\mathrm{d}\left(\mathrm{i}_{\mathcal{X}_{t}} \omega_{t}\right)$. Since $\left[\omega_{t}\right]$ is constant, by Hodge theory on compact manifolds there is a smooth family of 1 -forms $\left\{\sigma_{t}\right\}_{t \in[0,1]}$ such that $\frac{\mathrm{d}}{\mathrm{d} t} \omega_{t}=\mathrm{d} \sigma_{t}$. By (2.4), $\varphi_{t}^{*} \mathrm{~d}\left(\sigma_{t}+\mathrm{i}_{\mathcal{X}_{t}} \omega_{t}\right)=0$. Choosing $\mathcal{X}_{t}$ to be determined by

$$
\sigma_{t}+\mathrm{i}_{\mathcal{X}_{t}} \omega_{t}=0
$$

by compactness we can integrate $\mathcal{X}_{t}$ and determine $\left\{\varphi_{t}\right\}_{t \in[0,1]}$. An extension of Moser's result to certain noncompact symplectic manifolds, under certain constraints, appears in 30.

2.5. Lagrangian submanifolds. In the early 1970s, Weinstein proved two theorems [158, 159] about what Maslov [112] called Lagrangian submanifolds, and which were influential in the development of symplectic geometry. We discuss them next.

If $V$ is a subspace of a symplectic vector space $(W, \omega)$, its symplectic complement $V^{\omega}$ consists of the $w \in W$ such that $\omega(w, v)=0$ for all $v \in V ; V$ is isotropic if $V \subseteq V^{\omega}$ (i.e., $\left.\omega\right|_{V \times V}=0$ ) and Lagrangian if $V=V^{\omega}$. If $V$ is isotropic, $2 \operatorname{dim} V \leqslant$ $\operatorname{dim} W$. If $V$ is Lagrangian, $2 \operatorname{dim} V=\operatorname{dim} W$.

Definition 2.22. A submanifold $L$ of a symplectic manifold $(M, \omega)$ is isotropic if $\mathrm{T}_{x} L$ is an isotropic subspace of $\left(\mathrm{T}_{x} M, \omega_{x}\right)$ for every $x \in L$, and it is Lagrangian if $\mathrm{T}_{x} L$ is a Lagrangian subspace of $\left(\mathrm{T}_{x} M, \omega_{x}\right)$ for every $x \in L$.

The submanifold $L$ is Lagrangian if and only if it is isotropic and $2 \operatorname{dim} L=$ $\operatorname{dim} M$. For instance, the set of $\left(x_{1}, y_{1}, \ldots, x_{n}, y_{n}\right) \in \mathbb{R}^{2 n}$ such that $x_{i}=c_{i}, c_{i} \in \mathbb{R}$, $i=1, \ldots, n$ is a Lagrangian submanifold of $\left(\mathbb{R}^{2 n}, \sum_{i=1}^{n} \mathrm{~d} x_{i} \wedge \mathrm{d} y_{i}\right)$. Cotangent bundles are a source of Lagrangian submanifolds: if $X$ is a smooth manifold, the image of a section $s: X \rightarrow \mathrm{T}^{*} X$ of $\mathrm{T}^{*} X$ is Lagrangian if and only if $\mathrm{d} s=0$.

Let $(M, \omega)$ be any symplectic manifold, and let $L$ be a compact Lagrangian submanifold of $(M, \omega)$. For any set $A \subset M$, we denote by $i_{L, A}: L \hookrightarrow A$ the inclusion of $L$ into $A$. Weinstein proved [158] that if $\omega^{\prime}$ is another symplectic form on $M$ for which $L$ is Lagrangian, then there are neighborhoods $U, V$ of $L$ and a diffeomorphism $\varphi: U \rightarrow V$ such that $i_{L, V}=\varphi \circ i_{L, U}$ and $\varphi^{*} \omega^{\prime}=\omega$. This result is known as the Lagrangian neighborhood theorem and, using it, Weinstein proved the Weinstein tubular neighborhood theorem, which we state next. Recall that a cotangent bundle comes equipped with a canonical symplectic form (Proposition 2.3).

Theorem 2.23 (Weinstein [158]). Let $j: L \rightarrow \mathrm{T}^{*} L$ be the embedding given by the zero section. Let $\omega_{\mathrm{T} * L}$ be the canonical cotangent bundle symplectic form on $\mathrm{T}^{*} L$. Then there exist neighborhoods $V_{0}$ of $L$ in $\mathrm{T}^{*} L$, and $V$ of $L$ in $M$, and a diffeomorphism $\phi: V_{0} \rightarrow V$ such that $i_{L, M}=\phi \circ j$ and $\phi^{*} \omega=\omega_{\mathrm{T}{ }^{*} L}$. That is, any compact Lagrangian submanifold may be viewed as the zero section of its cotangent bundle, and it has a neighborhood which is symplectomorphic to a neighborhood of this zero section, with respect to the canonical cotangent bundle symplectic form.

As we will see, Lagrangian submanifolds play a central role in the theory of symplectic group actions (and in other parts of symplectic geometry-for instance the study of intersections of Lagrangian submanifolds is an important research area; see Arnold [5], Chaperon [24, and Hofer [79]). 
2.6. Monotonic symplectic invariants. Let $\mathrm{B}^{2 n}(r)$ be the $2 n$-ball of radius $r>0$ in $\mathbb{R}^{2 n}$ and let

$$
\mathrm{Z}^{2 n}(r):=\left\{\left(x_{1}, y_{1}, \ldots, x_{n}, y_{n}\right) \mid\left(x_{1}\right)^{2}+\left(y_{1}\right)^{2}<r^{2}\right\} \subset \mathbb{R}^{2 n}
$$

be the $2 n$-cylinder of radius $r$. They both inherit the symplectic form $\omega_{\mathbb{R}^{2 n}}=$ $\sum_{i=1}^{n} \mathrm{~d} x_{i} \wedge \mathrm{d} y_{i}$ from $\mathbb{R}^{2 n}$.

If $U, V$ are open subsets of $\mathbb{R}^{2 n}$, a symplectic embedding $f: U \hookrightarrow V$ is a smooth embedding such that $f^{*} \omega_{\mathbb{R}^{2 n}}=\omega_{\mathbb{R}^{2 n}}$. Similarly one defines symplectic embeddings $f:(M, \omega) \hookrightarrow\left(M^{\prime}, \omega^{\prime}\right)$ between general symplectic manifolds.

If there is a symplectic embedding $f: U \hookrightarrow V$, then the volume of $U$ is at most equal to the volume of $V$, that is, the volume provides an elementary embedding obstruction. Moreover:

Theorem 2.24 (Gromov [66]). There is no symplectic embedding of $\mathrm{B}^{2 n}(1)$ into $\mathrm{Z}^{2 n}(r)$ for $r<1$.

This important result exhibits a rigidity property of symplectic transformations. It shows that in addition to the volume there are subtler obstructions that come from the symplectic form. These obstructions can be formalized using the notion of symplectic capacity, which we discuss next.

Denote by $\mathcal{E}$ the category of ellipsoids in $\mathbb{R}^{2 n}$,

$$
\sum_{i=1}^{n} \frac{\left(x_{i}\right)^{2}+\left(y_{i}\right)^{2}}{r_{i}^{2}} \leqslant 1, \quad r_{i}>0, \quad i=1, \ldots, n,
$$

with symplectic embeddings induced by global symplectomorphisms of $\mathbb{R}^{2 n}$ as morphisms; by allowing some $r_{i}$ to be $\infty, \mathcal{E}$ contains products $\mathrm{B}^{2 d}(1) \times \mathbb{R}^{2(n-d)}$, $1 \leqslant d \leqslant n$. A symplectic category [27] is a subcategory $\mathcal{C}$ of the category of symplectic manifolds of dimension $2 n$, with symplectic embeddings as morphisms, containing $\mathcal{E}$ and such that $(M, \omega) \in \mathcal{C}$ implies that $(M, \lambda \omega) \in \mathcal{C}$ for all $\lambda>0$.

Definition 2.25. Let $d$ be an integer, $1 \leqslant d \leqslant n$. A symplectic d-capacity on a symplectic category $\mathcal{C}$ is a functor $c: \mathcal{C} \rightarrow([0, \infty], \leqslant)$ satisfying that $c(M, \omega) \leqslant$ $c\left(M^{\prime}, \omega^{\prime}\right)$ if there is a morphism from $(M, \omega)$ to $\left(M^{\prime}, \omega^{\prime}\right)$ (monotonicity, this a reformulation of functoriality); $c(M, \lambda \omega)=\lambda c(M, \omega)$ for all $\lambda>0$ (conformality); and $c\left(\mathrm{~B}^{2 n}(1)\right)>0, c\left(\mathrm{~B}^{2 d}(1) \times \mathbb{R}^{2(n-d)}\right)<\infty$, and $c\left(\mathrm{~B}^{2(d-1)}(1) \times \mathbb{R}^{2(n-d+1)}\right)=\infty$ (nontriviality). If $d=1$, a symplectic $d$-capacity is called a symplectic capacity.

Symplectic $(d-)$ capacities were introduced in Ekeland and Hofer's work [44,80]. The first known symplectic capacity was the Gromov radius, constructed by Gromov in [66] on the category of $2 n$-dimensional symplectic manifolds: at a $2 n$-dimensional symplectic manifold $(M, \omega)$ it is the radius of the largest $2 n$-dimensional ball that can be symplectically embedded into $(M, \omega)$. The fact that this defines a symplectic capacity is a deep result; it follows from Theorem 2.24.

Today many constructions of symplectic capacities are known; see [27]. Therein one can find for instance two of the best-known symplectic capacities, the HoferZehnder capacities and the Ekeland-Hofer capacities, but there are many more. There are also invariants of symplectic manifolds which do not fit Definition 2.25. see for instance [116.

The symplectic volume (2.2) is a symplectic $n$-capacity, and the Gromov radius is a symplectic 1-capacity. For many years it was unknown whether symplectic $d$-capacities could exist for intermediate values of $d$, that is, whether there were 
intermediate monotonic invariants measuring $d$-dimensional information about a symplectic manifold of dimension $2 n$ (this was a question of Hofer [80]). In the following, a symplectic $d$-capacity satisfies the exhaustion property if the value of the capacity on any open set equals the supremum of the values on its compact subsets.

Theorem 2.26 (Guth [78]). If $n \geqslant 3$, symplectic d-capacities satisfying the exhaustion property do not exist on any symplectic category $\mathcal{C}$ of $2 n$-dimensional manifolds for $2 \leqslant d \leqslant n-1$.

The assumption (exhaustion property) in Guth's theorem was removed in 139; so, other than volume, the monotonic invariants of symplectic geometry only measure two-dimensional information (which answers Hofer's question). As explained therein, one of the steps in [78, 139] generalizes an idea of Polterovich.

An equivariant theory of symplectic capacities has been given in [51]; we will discuss it later.

\section{Symplectic And Hamiltonian aCtions}

Since Lie groups are simultaneously groups and smooth manifolds, they naturally act on other smooth manifolds and describe their symmetries. In symplectic geometry, the smooth manifold is also symplectic, and the Lie group actions of interest preserve the symplectic structure (called symplectic actions); an important subclass of these (called Hamiltonian) admit in addition a so-called momentum map. The goal of this section is to introduce the basic theory of symplectic and Hamiltonian actions. Lie groups and Lie group actions are named after Sophus Lie (1842-1899), one of the most influential figures in differential geometry and back to whom many modern notions may be traced (including particular cases of the momentum map, which we will define shortly).

3.1. Lie group actions. Let $M$ be a smooth manifold, and let $G=(G, \star)$ be a Lie group with identity $e$. A smooth $G$-action on $M$ is a smooth map $G \times M \rightarrow M$, denoted by $(g, x) \mapsto g \cdot x$, such that $e \cdot x=x$ and $g \cdot(h \cdot x)=(g \star h) \cdot x$, for all $g, h \in G$ and for all $x \in M$; for simplicity sometimes we write $g h$ instead of $g \star h$ (or $g \cdot h$ ). For instance, we have the following smooth actions. Let $S^{1}$ be the circle, which may be viewed in two isomorphic ways - either as a quotient of $\mathbb{R}$ by its integral lattice $\mathbb{Z}$, or as a subset of the complex numbers $(\mathbb{R} / \mathbb{Z},+) \simeq\left(S^{1}:=\{z \in \mathbb{C}|| z \mid=1\}, \cdot\right)$. The map $S^{1} \times \mathbb{C}^{n} \rightarrow \mathbb{C}^{n}$ on $\mathbb{C}^{n},\left(\theta,\left(z_{1}, z_{2}, \ldots, z_{n}\right)\right) \mapsto\left(\theta z_{1}, z_{2}, \ldots, z_{n}\right)$, is a smooth $S^{1}$ action on $\mathbb{C}^{n}$. Also, any Lie group $G$ acts on itself by left multiplication $g \mapsto g h$ and analogously right multiplication, and also by the adjoint action $\operatorname{Ad}(h): g \mapsto h g h^{-1}$.

We say that the $G$-action is effective if every element in $T$ moves at least one point in $M$, or equivalently $\bigcap_{x \in M} G_{x}=\{e\}$, where $G_{x}:=\{t \in G \mid t \cdot x=x\}$ is the stabilizer subgroup of the $G$-action at $x$. The action is free if $G_{x}=\{e\}$ for every $x \in M$. The action is semifree if for every $x \in M$ either $G_{x}=G$ or $G_{x}=\{e\}$. The action is proper if the map $G \times M \rightarrow M \times M$ given by $(g, x) \mapsto(g \cdot x, x)$ is proper; if $G$ is compact, any smooth $G$-action on a smooth manifold is proper. The set $G \cdot x:=\{t \cdot x \mid t \in G\}$ is the $G$-orbit that goes through the point $x$.

Proposition 3.1. The stabilizer subgroup $G_{x}$ of a smooth $G$-action is a Lie subgroup of $G$. 
Proof. Let $f$ be the composite $G \rightarrow G \times M, g \mapsto(g, x)$, and the smooth action map $G \times M \rightarrow M$. Then $G_{x}=f^{-1}(\{x\})$. A famous theorem of Cartan 23] says that a closed subgroup of a Lie group is a Lie subgroup. Since $f$ is continuous, the result follows from Cartan's theorem.

For each closed subgroup $H$ of a Lie group $G$ which can occur as a stabilizer subgroup, the orbit type $M^{H}$ is defined as the set of all $x \in M$ such that $G_{x}$ is conjugate to $H$. Each connected component of $M^{H}$ is a smooth $G$-invariant submanifold of $M$. The connected components of the orbit types in $M$ form a Whitney stratification, called the orbit type stratification. There is a unique open orbit type, called the principal orbit type, denoted by $M_{\mathrm{reg}}$, which is the orbit type of a subgroup $H$ which is contained in every $G_{x}, x \in M$. A point $x \in M_{\text {reg }}$ is regular. The principal orbits are the orbits in $M_{\text {reg }}$.

If the $G$-action is effective, $M_{\text {reg }}$ consists of the points $x$ at which the action is free. It is a consequence of Duistermaat and Kolk [39, Theorem 2.8.5] that if a smooth $G$-action on a smooth manifold $M$ is proper, then $M_{\text {reg }}$ is an open dense subset of $M$.

Let $\cdot: G \times M \rightarrow$ and $\bullet^{\prime}: G \times M \rightarrow M$ be smooth $G$-actions, and let $\varphi: M \rightarrow$ $M^{\prime}$ be a smooth map. The map $\varphi$ is a G-equivariant diffeomorphism if it is a diffeomorphism and $\varphi(g \cdot x)=g \cdot^{\prime} \varphi(x)$ for all $g \in G, x \in M$. Similarly one defines a $G$-equivariant embedding.

Proposition 3.2. The stabilizer subgroup $G_{x}$ of a proper smooth $G$-action on a manifold $M$ is compact and $g \mapsto g \cdot x$ induces a smooth $G$-equivariant embedding $\alpha_{x}: G / G_{x} \rightarrow M$ with image $G \cdot x$.

In 166. Yau gives obstructions to the existence of smooth actions. For example [166, Corollary, p. 242] implies that if a compact connected Lie group $G$ acts effectively on an $n$-dimensional manifold $M$ and there are $\sigma_{1}, \ldots, \sigma_{n} \in \mathrm{H}^{1}(M ; \mathbb{Q})$ such that $\sigma_{1} \cup \cdots \cup \sigma_{n} \neq 0$, then $G$ is Abelian.

3.2. The infinitesimal action. Let $T$ be a a compact connected Abelian Lie group of dimension $n$. In fact, one can show [39. Corollary 1.12.4] that $T$ is a torus, i.e., $T$ is isomorphic to a product of $n$ circles

$$
T \simeq\left((\mathbb{R} / \mathbb{Z})^{n},+\right) \simeq\left(\left(S^{1}\right)^{n}:=\{z \in \mathbb{C}|| z \mid=1\}^{n},(\cdot, \ldots, \cdot)\right) .
$$

Let 1 be the identity in $T$, and let $\mathfrak{t}:=\mathrm{T}_{1} T$ be the Lie algebra of $T$. Let $X \in \mathfrak{t}$. There exists a unique homomorphism $\alpha_{X}: \mathbb{R} \rightarrow T$ with $\alpha_{X}(0)=1, \alpha_{X}^{\prime}(0)=X$. Define the exponential mapping exp: $\mathfrak{t} \rightarrow T$ by

$$
\exp (X):=\alpha_{X}(1) \text {. }
$$

The exponential mapping exp $: \mathfrak{t} \rightarrow T$ is a surjective homomorphism from the additive Lie group $(\mathfrak{t},+)$ onto $T$. Furthermore, $\mathfrak{t}_{\mathbb{Z}}:=\operatorname{ker}(\exp )$ is a discrete subgroup of $(\mathfrak{t},+)$, and exp induces an isomorphism from $\mathfrak{t} / \mathfrak{t}_{\mathbb{Z}}$ onto $T$, which we also denote by exp. The set $\mathfrak{t}_{\mathbb{Z}}$ is called the integral lattice.

Because $\mathfrak{t} / \mathfrak{t}_{\mathbb{Z}}$ is compact, $\mathfrak{t}_{\mathbb{Z}}$ has a $\mathbb{Z}$-basis which also an $\mathbb{R}$-basis of $\mathfrak{t}$, and each $\mathbb{Z}$-basis of $\mathfrak{t}_{\mathbb{Z}}$ is an $\mathbb{R}$-basis of $\mathfrak{t}$. Using coordinates with respect to an ordered $\mathbb{Z}$-basis of $\mathfrak{t}_{\mathbb{Z}}$, we obtain a linear isomorphism from $\mathfrak{t}$ onto $\mathbb{R}^{n}$ which maps $\mathfrak{t}_{\mathbb{Z}}$ onto $\mathbb{Z}^{n}$ and induces an isomorphism from $T \rightarrow \mathbb{R}^{n} / \mathbb{Z}^{n}$.

Using (3.1), one can generate vector fields on a smooth manifold from a given action. 
Definition 3.3. For each $X \in \mathfrak{t}$, the vector field infinitesimal action $X_{M}$ of $X$ on $M$ is defined by

$$
X_{M}(x):=\text { tangent vector to } \underbrace{t \mapsto \overbrace{\exp (t X)}^{\text {curve in } T} \cdot x}_{\text {curve in } M \text { through } x} \text { at } t=0,
$$

i.e., $X_{M}(x)=\mathrm{d} /\left.\mathrm{d} t\right|_{t=0} \exp (t X) \cdot x$.

3.3. Symplectic and Hamiltonian actions: definitions. The key notion of this paper is the following, which is valid for any Lie group $G$. Let $(M, \omega)$ be a $2 n$-dimensional symplectic manifold.

Definition 3.4. Let $\phi: G \times M \rightarrow M$ be any smooth $G$-action. We say that the $G$-action is symplectic if $G$ acts by symplectomorphisms, i.e., for each $t \in G$ the diffeomorphism $\phi_{t}: M \rightarrow M$ given by $\phi_{t}(x):=t \cdot x$ is such that $\left(\phi_{t}\right)^{*} \omega=\omega$. The triple $(M, \omega, \phi)$ is a called a symplectic $G$-manifold.

Often we write $(M, \omega, G)$ instead of $(M, \omega, \phi)$. Unless otherwise stated, from now on $G=T$ is compact, connected, and Abelian, i.e., a torus. By (2.3) applied to $\mathcal{X}=X_{M}$, the fact that the $T$-action is symplectic says that

$$
\mathrm{d}\left(\mathrm{i}_{X_{M}} \omega\right)=\mathrm{L}_{X_{M}} \omega=0, \quad \forall X \in \mathfrak{t},
$$

since $\mathrm{d} \omega=0$. The case when $\mathrm{i}_{X_{M}} \omega$ is, moreover, an exact form for each $X \in \mathfrak{t}$, and has been thoroughly studied in the literature. Indeed, there is a special type of symplectic action which appears often in classical mechanics and which enjoys a number of interesting properties.

Definition 3.5. Let $\mathfrak{t}^{*}$ be the dual of $\mathfrak{t}$. A symplectic action $T \times M \rightarrow M$ is Hamiltonian if there is a smooth map $\mu: M \rightarrow \mathfrak{t}^{*}$ such that Hamilton's equation

$$
-\mathrm{d}\langle\mu(\cdot), X\rangle=\mathrm{i}_{X_{M}} \omega, \quad \forall X \in \mathfrak{t},
$$

holds, where the left-hand side of equation (3.4) is the differential of the function $\langle\mu(\cdot), X\rangle: M \rightarrow \mathbb{R}$.

Hamiltonian actions are named after William Rowan Hamilton (1805-1865).

There is a natural notion of symplectic and Hamiltonian vector fields. Given a smooth function $f: M \rightarrow \mathbb{R}$, let $\mathcal{X}_{f}$ be the vector field defined by Hamilton's equation

$$
\omega\left(\mathcal{X}_{f}, \cdot\right)=-\mathrm{d} f .
$$

Definition 3.6. A smooth vector field $\mathcal{Y}$ on a symplectic manifold $(M, \omega)$ is symplectic if its flow preserves $\omega$, and Hamiltonian if there exists a smooth function $f: M \rightarrow \mathbb{R}$ such that $\mathcal{Y}=\mathcal{X}_{f}$.

A $T$-action on $(M, \omega)$ is symplectic if and only if all the vector fields that it generates through (3.2) are symplectic, and it is Hamiltonian if and only if all of these vector fields are Hamiltonian.

Proposition 3.7. Any symplectic T-action on a simply connected manifold $(M, \omega)$ is Hamiltonian.

Proof. The obstruction to $\mathrm{i}_{X_{M}} \omega$ being exact lies in the first cohomology group of the manifold $\mathrm{H}^{1}(M ; \mathbb{R})=0$. If the manifold is simply connected, then $\pi_{1}(M)=0$, and hence $\mathrm{H}^{1}(M ; \mathbb{R})=0$. 
Remark 3.8. For each $X \in \mathfrak{t}$, it follows from equation (3.4) that zeros of $X_{M}$ correspond to critical points of $\langle\mu(\cdot), X\rangle: M \rightarrow \mathbb{R}$, and $\langle\mu(\cdot), X\rangle$ always has critical points if $M$ is compact.

The natural transformations between symplectic manifolds $(M, \omega)$ and $\left(M^{\prime}, \omega^{\prime}\right)$ with symplectic $T$-actions are the $T$-equivariant diffeomorphisms which preserve the symplectic form, called T-equivariant symplectomorphisms. In the same way one defines $T$-equivariant symplectic embeddings.

In certain situations, for instance when working on equivariant symplectic packing problems [51,52, 126, 127, 133, it is useful to work with equivariant symplectic embeddings or symplectomorphisms up to reparametrizations of the acting group. In this case the equivariant condition (see Proposition 3.2 and the paragraph before it) states that $\varphi(g \cdot x)=f(g) \cdot^{\prime} \varphi(x)$ for some Lie group isomorphism $f: G \rightarrow G^{\prime}$.

Remark 3.9. Kostant [97] and Souriau [149] gave the general notion of a momentum map (see Marsden and Ratiu [110, pp. 369, 370] for the history). The momentum map may be defined for a Hamiltonian action of any Lie group. It was a key tool in Kostant [98] and Souriau discussed it at length in [150. We only deal with the momentum map for a Hamiltonian torus action.

3.4. Conditions for a symplectic action to be Hamiltonian. A Hamiltonian $S^{1}$-action on a compact symplectic $2 n$-dimensional manifold $(M, \omega)$ has at least $n+1$ fixed points. This is due to the fact that, if the fixed point set is discrete, the momentum map is a perfect Morse function whose critical set is the fixed point set. Hence the number of fixed points is $\sum_{i=0}^{2 n} \operatorname{rank} \mathrm{H}_{i}(M ; \mathbb{R})$, so at least $n+1$ since $[1],[\omega],\left[\omega^{2}\right], \ldots,\left[\omega^{n}\right]$ are nontrivial cohomology classes (Proposition 2.10).

We are not aware of general criteria to detect when a symplectic action is Hamiltonian, other than in a few specific situations. In fact, one striking question follows.

Question 3.10. Are there non-Hamiltonian symplectic $S^{1}$-actions on compact connected symplectic manifolds with nonempty discrete fixed point sets?

In recent years there has been a flurry of activity related to this question; see for instance Godinho 62, 63, Jang [85, 86, Pelayo and Tolman [135, and Tolman and Weitsman [155]. Tolman and Weitsman proved that the answer to the question is "no" for semifree actions (they used equivariant cohomological methods, covered here in Section 4.4); see Section 3.1 for the notion of semifree action.

Theorem 3.11 (Tolman and Weitsman [155). Let $(M, \omega)$ be a compact connected symplectic manifold, equipped with a semifree symplectic $S^{1}$-action with isolated fixed points. If there is at least one fixed point, the $S^{1}$-action is Hamiltonian.

In the Kähler case the answer to the question is a classical result of Frankel, which started much of the activity in the subject.

Theorem 3.12 (Frankel [57]). Let $(M, \omega)$ be a compact connected Kähler manifold admitting an $S^{1}$-action preserving the Kähler structure. If the $S^{1}$-action has some fixed point, it is Hamiltonian.

Ono [19] proved the analogue of Theorem 3.12 for compact Lefschetz manifolds, and McDuff [115, Proposition 2] proved a symplectic version (later generalized by Kim 93]).

Theorem 3.13 (McDuff [115]). A symplectic $S^{1}$-action on a compact connected symplectic 4-manifold $(M, \omega)$ with some fixed point is Hamiltonian. 
McDuff [115, Proposition 1] also constructed a compact connected symplectic 6 -manifold with a non-Hamiltonian symplectic $S^{1}$-action with fixed point set equal to a union of tori.

Recently Tolman has answered Question 3.10 in the positive:

Theorem 3.14 (Tolman [154]). There exists a symplectic non-Hamiltonian $S^{1}$-action on a compact connected manifold with exactly 32 fixed points.

Less is known for higher-dimensional Lie groups; the following corresponds to [60, Theorem 3.13].

Theorem 3.15 (Giacobbe 60]). An effective symplectic action of an $n$-dimensional torus on a compact connected symplectic $2 n$-dimensional manifold with some fixed point must be Hamiltonian.

Theorem 3.15 appears as [40, Corollary 3.9]. If $n=2$, this is deduced from [128, Theorem 8.2.1] (Theorem 8.15 later in this paper) in view of [42, Theorem 1.1].

There are results by Ginzburg describing the obstruction to the existence of a momentum map for a symplectic action (see 61]) where he showed that a symplectic action can be decomposed as a cohomologically free action and a Hamiltonian action.

Later we will give recent classifications of certain classes of symplectic actions, for which a complete answer to the following question may be given in terms of certain invariants.

Question 3.16. When is a symplectic torus action on a compact connected symplectic manifold Hamiltonian? Describe precisely the obstruction to being Hamiltonian.

3.5. Monotonic symplectic $G$-invariants. Let us now discuss an equivariant analogue of symplectic capacities (Section 2.6). The notion of symplectic $G$-action is valid in general for any Lie group, not necessarily a torus; see Definition 3.4 .

Let $\mathcal{M}_{G}$ be the set of $2 n$-dimensional symplectic $G$-manifolds; it is a category with morphisms given by $G$-equivariant symplectic embeddings.

A subcategory $\mathcal{C}$ of $\mathcal{M}_{G}$ a symplectic $G$-category if $(M, \omega) \in \mathcal{C}$ implies $(M, \lambda \omega) \in$ $\mathcal{C}$ for any $\lambda>0$.

Definition 3.17. A generalized symplectic $G$-capacity is a map $c: \mathcal{C} \rightarrow[0, \infty]$ such that if there is a $G$-equivariant symplectic embedding from $M$ to $M^{\prime}, M, M^{\prime} \in \mathcal{C}$, then $c(M) \leqslant c\left(M^{\prime}\right)$ (monotonicity), and if $\lambda>0, M \in \mathcal{C}$, then $c(M, \lambda \omega)=\lambda c(M, \omega)$ (conformality).

The nontriviality condition (Definition 2.25) has to be modified to make sense in the equivariant setting, as follows. We say that $c$ is tamed by $N \in \mathcal{M}_{G}$ if there is $t \in(0, \infty)$ such that if $M \in \mathcal{C}$ and there exists a $G$-equivariant symplectic embedding from $M$ to $N$, then $c(M) \leqslant t$, and if $P \in \mathcal{C}$ and there exists a $G$ equivariant symplectic embedding from $N$ to $P$, then $t \leqslant c(P)$.

In 51 a generalization of the Gromov radius to the equivariant setting is given. Let $\mathbb{T}^{k}=(\mathbb{R} / \mathbb{Z})^{k}$. In order to do this, the authors introduce the notion of symplectic $\left(\mathbb{T}^{k} \times \mathbb{R}^{d-k}\right)$-capacity as a generalized symplectic $\left(\mathbb{T}^{k} \times \mathbb{R}^{d-k}\right)$-capacity that is, in addition, tamed by $\mathrm{B}^{2 n}(1)$ and $\mathrm{Z}^{2 n}(1)$ (where these are endowed with standard symplectic forms and actions). 
For $1 \leqslant k \leqslant m \leqslant n$, the $(m, k)$-equivariant Gromov radius, defined on $\mathcal{M}_{\mathbb{R}^{k}}$, is, at $(M, \omega)$, the radius of the largest ball $\mathrm{B}^{2 m}(r)$ that can be symplectically and $\mathbb{R}^{k}$ equivariantly embedded into $(M, \omega)$. Using elementary techniques, one can show that this defines a symplectic $\mathbb{R}^{k}$-capacity [51].

An example of interest in equivariant symplectic geometry may be constructed as follows, on the category of $2 n$-dimensional symplectic manifolds endowed with Hamiltonian $\mathbb{T}^{n}$-actions. First, a toric ball packing $P$ of $M$ is a disjoint collection of symplectically and $\mathbb{T}^{n}$-equivariantly embedded balls. Consider, for each $(M, \omega)$, the supremum of the set of $\sqrt[2 n]{f(P)}$ where $f(P)=\operatorname{vol}(P, \omega) / \operatorname{vol}\left(\mathrm{B}^{2 n}(1), \omega_{\mathbb{R}^{2 n}}\right)$. In [51] it is shown that this defines a symplectic $\mathbb{T}^{n}$-capacity.

In fact, symplectic $G$-capacities give a framework to study invariants of integrable systems. So far few such invariants are known beyond this case (and the semitoric case also discussed in [51]).

\section{Properties of Hamiltonian actions}

Hamiltonian actions have been studied extensively in the past 40 years, and there exists a rich theory with a number of striking results. Next we briefly discuss some highlights, focusing on Hamiltonian $T$-actions when $T$ is a torus. Let $\mathfrak{t}$ be the Lie algebra of $T$, and let $\mathfrak{t}^{*}$ be its dual Lie algebra.

4.1. Marsden-Weinstein-Meyer symplectic reduction. Even though one cannot in general take quotients of symplectic manifolds by group actions and get again a symplectic manifold, for Hamiltonian actions there is a notion of a symplectic quotient.

Theorem 4.1 (Marsden and Weinstein [111, Meyer [113]). Let $(M, \omega)$ be a symplectic manifold and suppose that the torus $T$ acts on it in a Hamiltonian fashion with momentum map $\mu: M \rightarrow \mathfrak{t}^{*}$. Let $i: \mu^{-1}(t) \hookrightarrow M$ be the inclusion map and suppose that $T$ acts freely on $\mu^{-1}(t)$. Then the orbit space

$$
M_{\text {red, } t}:=\mu^{-1}(t) / T
$$

is a smooth manifold, the projection $\pi: \mu^{-1}(t) \rightarrow M_{\mathrm{red}, t}$ is a principal T-bundle, and there is a symplectic form $\omega_{\mathrm{red}, t}$ on $M_{\mathrm{red}, t}$ such that $\pi^{*} \omega_{\mathrm{red}, t}=i^{*} \omega$.

See also [2, Section 4.3] for further discussion of this result. The result is also valid for any compact Lie group $G$ (not necessarily $G=T$ being a torus).

The fact that $T$ acts freely on $\mu^{-1}(t)$ implies that $t$ is a regular value of $\mu$ and hence the fiber $\mu^{-1}(t)$ is a closed $(\operatorname{dim} M-\operatorname{dim} T)$-dimensional submanifold of $M$.

Definition 4.2. The symplectic quotient $\left(M_{\mathrm{red}, t}, \omega_{\mathrm{red}, t}\right)$ is called the MarsdenWeinstein-Meyer symplectic reduction of $(M, \omega)$ for the $T$-action at $t$.

Symplectic reduction has numerous applications in mechanics and geometry; see for instance [110]. We will give an application in the proof of the upcoming result Theorem 6.4

4.2. Atiyah-Guillemin-Sternberg convexity. The Atiyah-Guillemin-Sternberg convexity theorem $(1982,[10,75]$ ) says that the image of the momentum map $\mu(M)$ is a convex polytope. 
Theorem 4.3 (Atiyah [10, Guillemin and Sternberg [75]). If the torus $T$ acts on a compact connected symplectic manifold $(M, \omega)$ in a Hamiltonian fashion, the image $\mu(M)$ under the momentum map $\mu: M \rightarrow \mathfrak{t}^{*}$ is a convex polytope in $\mathfrak{t}^{*}$. Moreover, $\mu(M)$ is the convex hull of the image under $\mu$ of the fixed point set of the T-action.

Definition 4.4. The polytope $\mu(M)$ is called the momentum polytope of $M$.

The set $\mu^{-1}(t)$ is connected for any $t \in \mu(M)$; this is known as Atiyah's connectivity theorem.

Remark 4.5. If $x \in M, \mu$ is constant at $O:=T \cdot x$, so if $x^{\prime} \in O, T_{x^{\prime}} O \subseteq$ $\operatorname{ker}\left(\mathrm{d} \mu_{x^{\prime}}: T_{x^{\prime}} M \rightarrow \mathfrak{t}^{*}\right)=\left(T_{x^{\prime}} O\right)^{\omega_{x}}$, hence $O$ is isotropic.

One precedent of the convexity theorem appears in Kostant's article 99]. Other convexity theorems were proven later by Birtea, Ortega, and Ratiu [20, Kirwan [95] (in the case of compact, non-Abelian group actions), Benoist [15], and Giacobbe 60], to name a few.

Convexity for Poisson actions has been studied by Alekseev, Flaschka and Ratiu, Ortega and Ratiu, and Weinstein [4,49,121,163, among others.

4.3. Duistermaat-Heckman theorems. At about the time when Atiyah and Guillemin and Sternberg proved the convexity result, Duistermaat and Heckman did influential work, which we describe next.

Let $(M, \omega)$ be a $2 n$-dimensional symplectic manifold, and let $T$ be an $m$-dimensional torus. Suppose that $T$ acts on $(M, \omega)$ in a Hamiltonian fashion with momentum map $\mu: M \rightarrow \mathfrak{t}^{*}$. Assume that $\mu$ is proper, that is, for every compact $K \subseteq \mathfrak{t}^{*}$, the preimage $\mu^{-1}(K)$ is compact.

Definition 4.6. The Liouville measure of a Borel subset $E$ of $M$ is $m_{\omega}(E):=$ $\frac{1}{n !} \int_{E} \omega^{n}$. The Duistermaat-Heckman measure $m_{\mathrm{DH}}$ on $\mathfrak{t}^{*}$ is the pushforward measure $\mu_{*} m_{\omega}$ of $m_{\omega}$ by $\mu$, i.e.,

$$
m_{\mathrm{DH}}(U)=\frac{1}{n !} \int_{\mu^{-1}(U)} \omega^{n} .
$$

Let $\lambda$ be the Lebesgue measure in $\mathfrak{t}^{*} \simeq \mathbb{R}^{m}$.

Theorem 4.7 (Duistermaat and Heckman [37, 38]). There is a function $f: \mathfrak{t}^{*} \rightarrow \mathbb{R}$ such that $f$ is a polynomial of degree at most $n-m$ on each component of regular values of $\mu$, and

$$
m_{\mathrm{DH}}(U)=\int_{U} f \mathrm{~d} \lambda .
$$

Definition 4.8. The function $f$ is called the Duistermaat-Heckman polynomial.

In the upcoming Example 5.1 we will find $f$ explicitly.

If $T$ acts freely on $\mu^{-1}(0)$, then it acts freely on fibers $\mu^{-1}(t)$ for which $t \in \mathfrak{t}^{*}$ is close to 0. Consider the Marsden-Weinstein-Meyer reduced space $M_{\text {red, } t}=$ $\mu^{-1}(t) / T$ (see Section 4.1 or [2, Section 4.3]) with the reduced symplectic form $\omega_{\text {red, } t}$.

Theorem 4.9 (Duistermaat and Heckman [37, 38]). The cohomology class $\left[\omega_{\text {red }, t}\right]$ varies linearly in $t$.

Theorem 4.9 does not hold for nonproper momentum maps; see [131, Remark $4.5]$. 
4.4. Atiyah-Bott-Berline-Vergne localization. A useful tool to study symplectic $S^{1}$-actions is equivariant cohomology because it encodes well-fixed point-set information. It can, in particular, help to detect when a symplectic action is Hamiltonian; for instance, it was used by Tolman and Weitsman to study when a semifree symplectic $S^{1}$-action is Hamiltonian (Theorem 3.11).

Within symplectic geometry, equivariant cohomology is an active area. We only give the basic definition, a foundational result, and an application. Although equivariant cohomology may be defined generally, we focus on $S^{1}$-equivariant cohomology. Let $S^{1}$ act on a smooth manifold $M$.

Definition 4.10. The equivariant cohomology of $M$ is $\mathrm{H}_{S^{1}}^{*}(M):=\mathrm{H}^{*}\left(M \times_{S^{1}} S^{\infty}\right)$.

For example, if $x$ is a point, then $\mathrm{H}_{S^{1}}^{*}(x ; \mathbb{Z})=\mathrm{H}^{*}\left(\mathbb{C} P^{\infty} ; \mathbb{Z}\right)=\mathbb{Z}[t]$.

If $V$ is an equivariant vector bundle over $M$, the equivariant Euler class of $V$ is the Euler class of the vector bundle $V \times{ }_{S^{1}} S^{\infty}$ over $M \times_{S^{1}} S^{\infty}$. The equivariant Chern classes of equivariant complex vector bundles are defined analogously.

If $M$ is oriented and compact, then the projection $\pi: M \times{ }_{S^{1}} S^{\infty} \rightarrow \mathbb{C} P^{\infty}$ induces a natural pushforward map, denoted by $\int_{M}, \pi_{*}: \mathrm{H}_{S^{1}}^{i}(M ; \mathbb{Z}) \rightarrow \mathrm{H}^{i-\operatorname{dim} M}\left(\mathbb{C} P^{\infty} ; \mathbb{Z}\right)$. In particular $\pi_{*}(\alpha)=0$ for all $\alpha \in \mathrm{H}_{S^{1}}^{i}(M ; \mathbb{Z})$ when $i<\operatorname{dim} M$.

Let $M^{S^{1}}$ denote the fixed point set of the $S^{1}$-action. For a component $F$ of $M^{S^{1}}$ we denote by $\mathrm{e}_{S^{1}}\left(\mathrm{~N}_{F}\right)$ the equivariant Euler class of the normal bundle to $F$.

Theorem 4.11 (Atiyah and Bott [11], Berline and Vergne [18]). Fix $\alpha \in \mathrm{H}_{S^{1}}^{*}(M ; \mathbb{Q})$. As elements of $\mathbb{Q}(t)$,

$$
\int_{M} \alpha=\sum_{F \subset M^{S^{1}}} \int_{F} \frac{\left.\alpha\right|_{F}}{\mathrm{e}_{S^{1}}\left(\mathrm{~N}_{F}\right)},
$$

where the sum is over all fixed components F.

Next we give an application of this theorem to counting fixed points of symplectic $S^{1}$-actions. Let $\omega$ be a symplectic form on $M$, let $S^{1}$ act symplectically on $(M, \omega)$, and let $J: \mathrm{T} M \rightarrow \mathrm{T} M$ be a compatible almost complex structure. Let $x$ be an isolated fixed point of the $S^{1}$-action.

Suppose $\operatorname{dim} M=2 n$. There is an identification of $\mathrm{T}_{x} M$ with $\mathbb{C}^{n}$, where $S^{1}$ acts on $\mathbb{C}^{n}$ is by $\lambda \cdot\left(z_{1}, \ldots, z_{n}\right)=\left(\lambda^{\xi_{1}} z_{1}, \ldots, \lambda^{\xi_{n}} z_{n}\right)$; the nonzero integers $\xi_{1}, \ldots, \xi_{n}$ are determined up to permutation by the $S^{1}$-action and symplectic form; they are the weights in the isotropy representation $\mathrm{T}_{x} M$. Let $\mathrm{c}_{1}(M)(x)$ and $\Lambda_{x}$ be the sum of the weights and the product of the weights at $x$, respectively.

Let $\sigma_{i}$ be the $i$ th elementary symmetric polynomial, and let $t$ be the generator of $\mathrm{H}_{S^{1}}^{2}(x ; \mathbb{Z})$. The restriction of the $i$ th-equivariant Chern class is $\left.\mathrm{c}_{i}(M)\right|_{x}=$ $\sigma_{i}\left(\xi_{1}, \ldots, \xi_{n}\right) t^{i}$. For instance, $\left.\mathrm{c}_{1}(M)\right|_{x}=\sum_{i=1}^{n} \xi_{i} t$ and

$$
\mathrm{e}_{S^{1}}\left(\mathrm{~N}_{x}\right)=\left.\mathrm{c}_{n}(M)\right|_{x}=\left(\prod_{j=1}^{n} \xi_{j}\right) t^{n} .
$$

Hence,

$$
\int_{x} \frac{\left.\mathrm{c}_{i}(M)\right|_{x}}{\mathrm{e}_{S^{1}}\left(\mathrm{~N}_{x}\right)}=\frac{\sigma_{i}\left(\xi_{1}, \ldots, \xi_{n}\right)}{\prod_{j=1}^{n} \xi_{j}} t^{i-n} .
$$

We identify $\left.\mathrm{c}_{1}(M)\right|_{x}$ with $\mathrm{c}_{1}(M)(x)$. 
Definition 4.12. The map $c_{1}(M): M^{S^{1}} \rightarrow \mathbb{Z}, x \mapsto c_{1}(M)(x) \in \mathbb{Z}$, is the Chern class map of $M$.

Proposition 4.13 ([135). If $S^{1}$ acts symplectically on the compact symplectic $2 n$-dimensional manifold $(M, \omega)$ with isolated fixed points, and the range of $\mathrm{c}_{1}(M): M^{S^{1}} \rightarrow \mathbb{Z}$ contains at most $n$ elements, then $\sum_{x \in\left(c_{1}(M)\right)^{-1}(k)}\left(\Lambda_{x}\right)^{-1}=0$ for every $k \in \mathbb{Z}$.

Proof. Let $\left\{\mathrm{c}_{1}(M)(x) \mid x \in M^{S^{1}}\right\}=\left\{k_{1}, \ldots, k_{\ell}\right\}$ and $A_{i}:=\sum_{x \in\left(c_{1}(M)\right)^{-1}\left(k_{i}\right)}\left(\Lambda_{x}\right)^{-1}$ for $1 \leqslant i \leqslant \ell$. Consider the $\ell \times \ell$ matrix $B$ given by $B_{i j}:=\left(k_{i}\right)^{j-1}$, where $1 \leqslant i, j \leqslant \ell$. Since $\ell \leqslant n$ by assumption, $\int_{M} \mathrm{c}_{1}(M)^{j}=0$ for all $j<\ell$. Applying Theorem 4.11 to the elements $1, \mathrm{c}_{1}(M), \ldots, \mathrm{c}_{1}(M)^{\ell-1}$ gives a homogenous system of linear equations $B \cdot\left(A_{1}, \ldots, A_{\ell}\right)=(0, \ldots, 0)$. Since $B$ is a Vandermonde matrix, $\operatorname{det}(B(\ell)) \neq 0$. Thus $A_{1}=\cdots=A_{\ell}=0$.

A map $f: X \rightarrow Y$ is somewhere injective if there is $y \in Y$ such that $f^{-1}(\{y\})$ is the singleton.

Theorem 4.14 ([135]). Let $S^{1}$ act symplectically on a compact symplectic $2 n$ manifold $(M, \omega)$ with isolated fixed points. If $c_{1}(M)$ is somewhere injective, the $S^{1}$-action has at least $n+1$ fixed points.

Proof. Since $c_{1}(M)$ is somewhere injective, there is $k \in \mathbb{Z}$ such that

$$
\sum_{x \in\left(c_{1}(M)\right)^{-1}(k)}\left(\Lambda_{x}\right)^{-1}=0
$$

By Proposition 4.13, this implies that the range $c_{1}(M)$ contains at least $n+1$ elements.

Using equivariant cohomology, other results are shown in [135] for instance, if $S^{1}$ acts symplectically on a compact symplectic manifold with exactly two fixed points, then $M$ is a 2 -sphere, or $\operatorname{dim} M=6$ and the weights at the fixed points are $a, b,-a-b$ or $a+b,-a-b$ ([135, Theorem 3]).

Another application of equivariant cohomology appeared in 64, where the authors give a lower bound for the number of fixed points of any symplectic $S^{1}$-action, under a mild assumption.

4.5. Further topics. There exists an extensive theory of Hamiltonian actions and related topics; see for instance the books by Guillemin 70, Guillemin and Sjamaar [74], and Ortega and Ratiu [121. There are several influential works which we do not describe here for two reasons - brevity is the main one, but also because they are more advanced and more suitable for a survey than a succinct invitation to the subject. These works include Sjamaar and Lerman's work [148] on stratifications, Kirwan's convexity theorem [95] (which generalizes the AtiyahGuillemin-Sternberg convexity theorem to the non-Abelian case), and Lerman's symplectic cutting [106] (a procedure to "cut" symplectic manifolds with applications in equivariant symplectic geometry and integrable systems). 


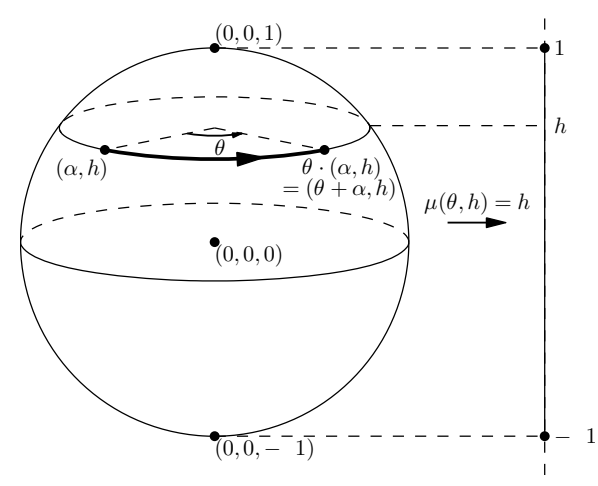

FiguRE 1. The unit sphere $S^{2}$ endowed with the standard area form $\omega=\mathrm{d} \theta \wedge \mathrm{d} h$ and rotational action of $S^{1}$ about the vertical axis, as explained in the first part of Example [5.1. This action has precisely two fixed points, the north and south poles of $S^{2}$. The momentum map is given by $\mu(\theta, h)=\theta$ for any $\theta \in S^{1}$ and $-1 \leqslant h \leqslant 1$. The $S^{1}$-orbits that are not fixed points are horizontal circles, and $\omega$ vanishes on them, so they are isotropic (in fact, Lagrangian), as in Remark 4.5 .

\section{Examples of Hamiltonian AND SympleCtiC ACTIONS}

5.1. Symplectic Hamiltonian actions. The following are examples of Hamiltonian actions.

Example 5.1. Let $\omega=\mathrm{d} \theta \wedge \mathrm{d} h$ be the standard area form in spherical coordinates on $S^{2}$. Endow $\left(S^{2}, \omega=\mathrm{d} \theta \wedge \mathrm{d} h\right)$ with the rotational $S^{1}$-action about the $z$-axis (Figure 11). Identifying the dual Lie algebra of $S^{1}$ with $\mathbb{R}$ by choosing a basis, the momentum map $\mu: S^{2} \rightarrow \mathbb{R}$ is $\mu(\theta, h)=h$, and the momentum polytope (Definition 4.4) is $\Delta=[-1,1]$. The Duistermaat-Heckman polynomial (Definition [4.8) is $f=2 \pi \chi_{[-1,1]}$ where $\chi_{[-1,1]}$ is the characteristic function of $[-1,1]$ so $m_{\mathrm{DH}}([a, b])=2 \pi(b-a)$ for any $[a, b] \subseteq[-1,1]$.

Example 5.2. Consider the $n$-dimensional complex projective space equipped with a $\lambda$-multiple, $\lambda>0$, of the Fubini-Study form $\left(\mathbb{C} P^{n}, \lambda \cdot \omega_{\mathrm{FS}}\right)$ and the rotational $\mathbb{T}^{n}$ action induced from the rotational $\mathbb{T}^{n}$-action on the $(2 n+1)$-dimensional complex plane. This is a Hamiltonian action with momentum map

$$
\left[z_{0}: z_{1}: \ldots, z_{n}\right] \mapsto\left(\lambda\left|z_{1}\right|^{2} / \sum_{i=0}^{n}\left|z_{i}\right|^{2}, \ldots, \lambda\left|z_{n}\right|^{2} / \sum_{i=0}^{n}\left|z_{i}\right|^{2}\right) .
$$

If $\mathrm{e}_{1}=(1,0, \ldots, 0) \in \mathbb{R}^{n}, \ldots, \mathrm{e}_{n}=(0, \ldots, 0,1) \in \mathbb{R}^{n}$, the momentum polytope is

$$
\Delta=\text { convex hull }\left\{0, \lambda \mathrm{e}_{1}, \ldots, \lambda \mathrm{e}_{n}\right\} .
$$

The category of Hamiltonian actions, while large, does not include some simple examples of symplectic actions, for instance free symplectic actions on compact manifolds, because Hamiltonian actions on compact manifolds always have fixed points (this is implied, for instance, by Theorem 4.3). 
5.2. Symplectic non-Hamiltonian actions. Theorem 3.14 concerns a non-Hamiltonian symplectic action with isolated fixed points; next we give examples with nonisolated fixed points.

5.2.1. Examples with symplectic orbits. In the following examples there is no momentum map; they are examples of what we later call maximal symplectic actions (discussed in Section 7); each orbit of such an action is a symplectic submanifold in the following sense. If $V$ is a subspace of a symplectic vector space $(W, \omega)$, it is symplectic if $V^{\omega} \cap V=\{0\}$ (i.e., $\left.\omega\right|_{V \times V}$ is nondegenerate).

Definition 5.3. A submanifold $C$ of a symplectic manifold $(M, \omega)$ is symplectic if $\mathrm{T}_{x} C$ is a symplectic subspace of $\left(\mathrm{T}_{x} M, \omega_{x}\right)$ for every $x \in C$.

If $i: C \hookrightarrow M$ is the inclusion, then $\left(C, i^{*} \omega\right)$ is a symplectic manifold. In the same spirit of the results of Section 2.5, a theorem of Weinstein [158] states that a neighborhood of $C$ is determined by the restriction of the symplectic form to the submanifold, together with the isomorphism class of its normal bundle (a symplectic vector bundle); see [116, pp. 100, 101] for details.

Remark 5.4. It can be shown that the fixed point set in the Atiyah-GuilleminSternberg convexity theorem (Theorem 4.3) is a finite union of connected symplectic submanifolds of $M$.

The $T$-orbits of a Hamiltonian action are isotropic (Remark 4.5), and hence if a symplectic $T$-action has symplectic orbits, it is not Hamiltonian; this is the case for the following examples.

Example 5.5. Let $(M, \omega):=\left((\mathbb{R} / \mathbb{Z})^{2 n}, \sum_{i=1}^{n} \mathrm{~d} x_{i} \wedge \mathrm{d} y_{i}\right)$ with coordinates $\left(x_{1}, y_{1}, \ldots\right.$, $\left.x_{n}, y_{n}\right)$, and let the 2 -torus $T:=(\mathbb{R} / \mathbb{Z})^{2}$ act by translation on the first two components $\left(x_{1}, y_{1}\right)$. This action is free, symplectic, and the orbits are symplectic and diffeomorphic to $T$.

Example 5.6. Let $(M, \omega):=\left((\mathbb{R} / \mathbb{Z})^{2} \times S^{2}, \mathrm{~d} x \wedge \mathrm{d} y+\mathrm{d} \theta \wedge \mathrm{d} h\right)$ and $T:=(\mathbb{R} / \mathbb{Z})^{2}$ act by translations on the $T$-factor (Figure 2). This action is free, symplectic, and the orbits are symplectic, diffeomorphic to $T$.

Example 5.7. Let $P:=S^{2} \times(\mathbb{R} / \mathbb{Z})^{2}$ equipped with the product area form of the standard symplectic form $\mathrm{d} \theta \wedge \mathrm{d} h$ on $S^{2}$ and the standard area $\mathrm{d} x \wedge \mathrm{d} y$ form on the torus $(\mathbb{R} / \mathbb{Z})^{2}$. The 2 -torus $T:=(\mathbb{R} / \mathbb{Z})^{2}$ acts freely by translations on the right factor of $P$. Let the finite group $\mathbb{Z} / 2 \mathbb{Z}$ act on $S^{2}$ by rotating each point horizontally by 180 degrees, and let $\mathbb{Z} / 2 \mathbb{Z}$ act on $(\mathbb{R} / \mathbb{Z})^{2}$ by the antipodal action on the first circle $\mathbb{R} / \mathbb{Z}$. The diagonal action of $\mathbb{Z} / 2 \mathbb{Z}$ on $P$ is free. Therefore, the quotient space $S^{2} \times_{\mathbb{Z} / 2 \mathbb{Z}}(\mathbb{R} / \mathbb{Z})^{2}$ is a smooth manifold. Let $M:=S^{2} \times_{\mathbb{Z} / 2 \mathbb{Z}}(\mathbb{R} / \mathbb{Z})^{2}$ be endowed with the symplectic form $\omega$ and the $T$-action inherited from the ones on $P$. The action of $T$ on $M$ is symplectic but not free, and the $T$-orbits are symplectic two-dimensional tori. The orbit space $M / T=S^{2} /(\mathbb{Z} / 2 \mathbb{Z})$ is a smooth orbifold with two singular points of order 2 , the south and north poles of $S^{2}$ (this orbifold plays a role in the classification of maximal symplectic actions, which we will see in Section 8.1).

5.2.2. Examples with Lagrangian or coisotropic orbits. The following are examples of what we later call coisotropic actions (discussed in Section [7); in particular, each orbit of such an action is a coisotropic submanifold, meaning the following. If $V$ is a subspace of a symplectic vector space $(W, \omega)$, it is coisotropic if $V^{\omega} \subseteq V$. If $V$ is coisotropic, then $2 \operatorname{dim} V \geqslant \operatorname{dim} W$. 
Definition 5.8. A submanifold $C$ of a symplectic manifold $(M, \omega)$ is coisotropic if $\mathrm{T}_{x} C$ is a coisotropic subspace of $\left(\mathrm{T}_{x} M, \omega_{x}\right)$ for every $x \in C$.

A submanifold $C$ of the symplectic manifold $(M, \omega)$ is Lagrangian if and only if it is both isotropic and coisotropic.

Example 5.9. The action of $T:=(\mathbb{R} / \mathbb{Z})^{2 n-1}$ on $M:=\left((\mathbb{R} / \mathbb{Z})^{2 n}, \sum_{i=1}^{n} \mathrm{~d} x_{i} \wedge \mathrm{d} y_{i}\right)$ with coordinates $\left(x_{1}, y_{1}, \ldots, x_{n}, y_{n}\right)$ by translation on the first $2 n-1$ components $\left(x_{1}, y_{1}, \ldots, x_{n-1}, y_{n-1}, x_{n}\right)$ is symplectic, free, and hence not Hamiltonian (for instance by Theorem 4.3 a Hamiltonian action on a compact manifold has fixed points). Its orbits are coisotropic submanifolds diffeomorphic to $T$. The action of $T^{\prime}:=(\mathbb{R} / \mathbb{Z})^{n}$ on $M$ by translations on $\left(x_{1}, \ldots, x_{n}\right)$ is free, symplectic, and its orbits are Lagrangian (hence also coisotropic) submanifolds diffeomorphic to $T^{\prime}$.

Example 5.10 (Kodaira [96] and Thurston [152]). An important example of a nonHamiltonian symplectic torus action with Lagrangian orbits is the Kodaira variety [96] (also known as the Kodaira-Thurston manifold [152]), which is a torus bundle over a torus constructed as follows. Consider the product symplectic manifold $\left(\mathbb{R}^{2} \times(\mathbb{R} / \mathbb{Z})^{2}, \mathrm{~d} x_{1} \wedge \mathrm{d} y_{1}+\mathrm{d} x_{2} \wedge \mathrm{d} y_{2}\right)$, where $\left(x_{1}, y_{1}\right) \in \mathbb{R}^{2}$ and $\left(x_{2}, y_{2}\right) \in(\mathbb{R} / \mathbb{Z})^{2}$. Consider the action of $\left(j_{1}, j_{2}\right) \in \mathbb{Z}^{2}$ on $(\mathbb{R} / \mathbb{Z})^{2}$ by the matrix group of

$$
\left(\begin{array}{cc}
1 & j_{2} \\
0 & 1
\end{array}\right)
$$

where $j_{2} \in \mathbb{Z}$ (notice that $j_{1}$ does not appear intentionally in the matrix). The quotient of this symplectic manifold by the diagonal action of $\mathbb{Z}^{2}$ gives rise to a compact connected symplectic 4-manifold, the Kodaira variety,

$$
(\mathrm{KT}, \omega):=\left(\mathbb{R}^{2} \times_{\mathbb{Z}^{2}}(\mathbb{R} / \mathbb{Z})^{2}, \mathrm{~d} x_{1} \wedge \mathrm{d} y_{1}+\mathrm{d} x_{2} \wedge \mathrm{d} y_{2}\right),
$$

on which $T:=\mathbb{R} / \mathbb{Z} \times \mathbb{R} / \mathbb{Z}$ acts symplectically and freely, where the first circle acts on the $x_{1}$-component, and the second circle acts on the $y_{2}$-component (one can check that this action is well defined). This is a free symplectic action with Lagrangian orbits.

Example 5.11. Consider $(M, \omega):=\left((\mathbb{R} / \mathbb{Z})^{2} \times S^{2}, \mathrm{~d} x \wedge \mathrm{d} y+\mathrm{d} \theta \wedge \mathrm{d} h\right)$. There is an action of $T:=\mathbb{R} / \mathbb{Z} \times \mathbb{R} / \mathbb{Z}$ on $(M, \omega)$, where the first circle of $T$ acts on the
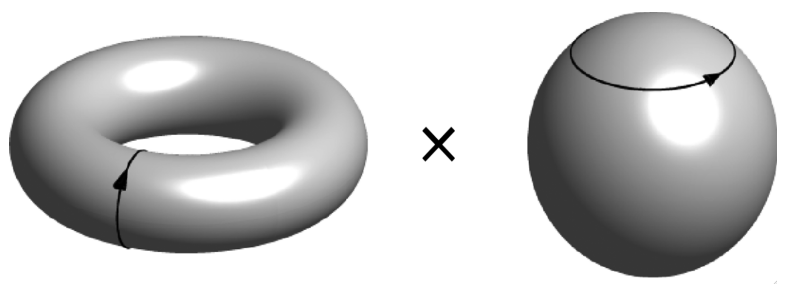

Figure 2. A symplectic 2-torus action on the product $(\mathbb{R} / \mathbb{Z})^{2} \times S^{2}$ of a 2 -torus and a 2 -sphere. The first circle of the acting 2-torus translates the first component of the first factor of the product. The second circle acts on the 2 -sphere by rotations about the $z$ axis (as described in Example 5.1 and Figure 1). This is a simple example of a symplectic action which is not Hamiltonian; see Example 5.11 
first circle of the left factor of $M$, and the right circle acts on $S^{2}$ by rotations about the $z$-axis; see Figure 2. This $T$-action is symplectic. However, it is not a Hamiltonian action because it does not have fixed points (the existence of which is implied, for Hamiltonian actions, for instance by Theorem 4.3). It is also not free, because the stabilizer subgroup of $(p, q)$, where $q$ is the north or south pole of $S^{2}$, is a circle. The principal orbits (Section 3.1) are products of the circle orbits of the left factor $(\mathbb{R} / \mathbb{Z})^{2}$, and the circle orbits of the right factor (all orbits of the right factor are circles but the north and south poles, which are fixed points). Because the principal orbits are obtained by keeping the $y$-coordinate on the left factor constant and the height on the right factor constant, we have that $d y=d h=0$, so they are Lagrangian.

\section{Classifications of Hamiltonian actions}

In a few cases there exist classifications of Hamiltonian $G$-actions on compact symplectic manifolds, in terms of symplectic invariants. In this section we discuss several classifications, beginning with the case in which $G$ is an $n$-dimensional torus acting on a $2 n$-dimensional manifold.

6.1. Classification of symplectic-toric manifolds. Let $T$ be an $n$-dimensional torus with Lie algebra $\mathfrak{t}$ and dual Lie algebra $\mathfrak{t}^{*}$.

6.1.1. Delzant polytopes. Let $\Delta$ be an $n$-dimensional convex polytope in $\mathfrak{t}^{*}$. Let $F$ be the set of codimension 1 faces of $\Delta$. Let $V$ be the set of vertices of $\Delta$. If $v \in V$, let $F_{v}:=\{f \in F \mid v \in f\}$. Following Guillemin [70, p. 8], we define a special type of polytope.

Definition 6.1. We say that $\Delta$ is a Delzant polytope if the following two conditions hold. First, for each $f \in F$ there are $X_{f} \in \mathfrak{t}_{\mathbb{Z}}$ and $\lambda_{f} \in \mathbb{R}$ such that the hyperplane which contains $f$ has defining equation $\left\langle X_{f}, \xi\right\rangle+\lambda_{f}=0, \xi \in \mathfrak{t}^{*}$, and $\Delta$ is contained in the set of $\xi \in \mathfrak{t}^{*}$ such that $\left\langle X_{f}, \xi\right\rangle+\lambda_{f} \geqslant 0$. Second, for every $v \in V,\left\{X_{f} \mid f \in F_{v}\right\}$ is a $\mathbb{Z}$-basis of $\mathfrak{t}_{\mathbb{Z}}$.

See Figure 3 for examples of Delzant polytopes. The definition implies that for each $f \in F$ there exists $X_{f} \in \mathfrak{t}_{\mathbb{Z}}$ and $\lambda_{f} \in \mathbb{R}$ such that

$$
\Delta=\left\{\xi \in \mathfrak{t}^{*} \mid\left\langle X_{f}, \xi\right\rangle+\lambda_{f} \geqslant 0, \forall f \in F\right\} .
$$

It follows that for every $v \in V$, the cardinality of $F_{v}$ is $n$.

If $z \in \mathbb{C}^{F}$ and $f \in F$, we write $z(f):=z_{f}$, which we view as the coordinate of $z$ with index $f$. Let $\pi: \mathbb{R}^{F} \rightarrow \mathfrak{t}$ be the linear map $\pi(t):=\sum_{f \in F} t_{f} X_{f}$. Because, for any vertex $v$ of the Delzant polytope $\Delta$, the $X_{f}$ with $f \in F_{v}$ form a $\mathbb{Z}$-basis of $\mathfrak{t}_{\mathbb{Z}}$ which is also an $\mathbb{R}$-basis of $\mathfrak{t}$, we have $\pi\left(\mathbb{Z}^{F}\right)=\mathfrak{t}_{\mathbb{Z}}$ and $\pi\left(\mathbb{R}^{F}\right)=\mathfrak{t}$. Hence $\pi$ induces a surjective Lie group homomorphism $\pi^{\prime}: \mathbb{R}^{F} / \mathbb{Z}^{F}=(\mathbb{R} / \mathbb{Z})^{F} \rightarrow \mathfrak{t} / \mathfrak{t}_{\mathbb{Z}}$, and hence a surjective homomorphism $\exp \circ \pi^{\prime}: \mathbb{R}^{F} / \mathbb{Z}^{F} \rightarrow T$. Write $\mathfrak{n}:=\operatorname{ker} \pi$ and

$$
N:=\operatorname{ker}\left(\exp \circ \pi^{\prime}\right),
$$

which is a compact Abelian subgroup of $\mathbb{R}^{F} / \mathbb{Z}^{F}$. Actually, $N$ is connected (see [41, Lemma 3.1]) and isomorphic to $\mathfrak{n} / \mathfrak{n}_{\mathbb{Z}}$, where $\mathfrak{n}_{\mathbb{Z}}:=\mathfrak{n} \cap \mathbb{Z}^{F}$ is the integral lattice in $\mathfrak{n}$ of the torus $N$. 

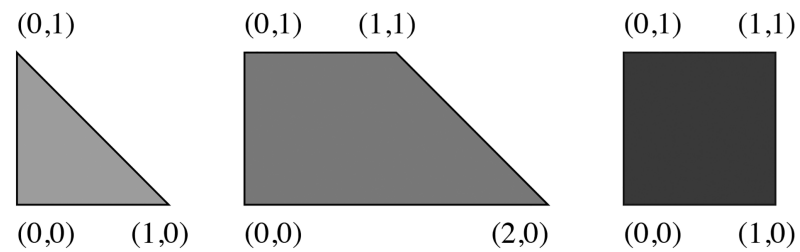

Figure 3. Examples of two-dimensional Delzant polytopes, i.e., Delzant polygons, as in Definition 6.1 Each of these polygons arises as the image under the momentum map of a compact connected symplectic 4-manifold with an effective Hamiltonian 2-torus action (the fact that these polygons uniquely determine the manifold is due to Theorem 6.4). In fact, the triangle corresponds to the standard $\mathbb{C} P^{2}$ (described in Example 5.2), the trapezoid to a Hirzebruch surface, and the square to $\mathbb{C} P^{1} \times \mathbb{C} P^{1}$.

6.1.2. Symplectic-toric manifolds. The following notion has been a source of inspiration to many authors working on symplectic and Hamiltonian group actions and integrable Hamiltonian systems.

Definition 6.2. A symplectic-toric manifold is a compact connected symplectic manifold $(M, \omega)$ of dimension $2 n$ endowed with an effective Hamiltonian action of a torus $T$ of dimension $n$.

Remark 6.3. The Duistermaat-Heckman polynomial $f$ (Definition 4.8) associated to the momentum map of a symplectic-toric manifold will be constant according to Theorem 4.7 (in fact, equal to the characteristic function of the image $\mu(M)$ of the momentum map).

Often, one denotes the symplectic-toric manifold by $(M, \omega, T)$; this is a particular example of a symplectic $T$-manifold (Definition 3.4).

The $S^{1}$-action on $S^{2}$ by rotations about the $z$-axis (Figure 1 and Example 5.1) is effective and Hamiltonian, and $\left(S^{2}, \mathrm{~d} \theta \wedge \mathrm{d} h, S^{1}\right)$ is a symplectic-toric manifold with momentum polytope $[-1,1]$. According to a striking result of Thomas Delzant, $[-1,1]$ completely characterizes $\left(S^{2}, \omega, S^{1}\right)$.

Theorem 6.4 (Delzant [33]). For every Delzant polytope $\Delta \subset \mathfrak{t}^{*}$ there exists a symplectic-toric manifold $\left(M_{\Delta}, \omega_{\Delta}, T\right)$ with momentum map $\mu_{\Delta}: M_{\Delta} \rightarrow \mathfrak{t}^{*}$ such that $\mu_{\Delta}\left(M_{\Delta}\right)=\Delta$. If $(M, \omega, T)$ is a symplectic-toric manifold with momentum map $\mu: M \rightarrow \mathfrak{t}^{*}$, then $\mu(M)$ is a Delzant polytope; moreover, $(M, \omega, T)$ is $T$ equivariantly symplectomorphic to the symplectic-toric manifold $\left(M_{\Delta}, \omega_{\Delta}, T\right)$ if, and only if, up to translations, $\mu(M)$ coincides with $\Delta$.

Proof. We will prove the existence part [33, pp. 328, 329] following [41, i.e., that for any Delzant polytope $\Delta$ there exists a symplectic-toric manifold $M_{\Delta}$ such that $\mu_{\Delta}\left(M_{\Delta}\right)=\Delta$, and which is obtained as the reduced phase space for a linear Hamiltonian action of the torus $N$ in (6.1) on a symplectic vector space $E$, at a value $\lambda_{N}$ of the momentum mapping of the Hamiltonian $N$-action, where $E, N$, and $\lambda_{N}$ are determined by $\Delta$.

On the complex vector space $\mathbb{C}^{F}$ of all complex-valued functions on $F$, we have the action of $\mathbb{R}^{F} / \mathbb{Z}^{F}$, where $t \in \mathbb{R}^{F} / \mathbb{Z}^{F}$ maps $z \in \mathbb{C}^{F}$ to the element $t \cdot z \in \mathbb{C}^{F}$ 
defined for $f \in F$ by $(t \cdot z)_{f}=\mathrm{e}^{2 \pi \mathrm{i} t_{f}} z_{f}$. The infinitesimal action of $Y \in \mathbb{R}^{F}=$ $\operatorname{Lie}\left(\mathbb{R}^{F} / \mathbb{Z}^{F}\right)$ is given by $(Y \cdot z)_{f}=2 \pi \mathrm{i} Y_{f} z_{f}$, which is a Hamiltonian vector field defined by the function

$$
z \mapsto\langle Y, \mu(z)\rangle=\sum_{f \in F} Y_{f} \frac{\left|z_{f}\right|^{2}}{2}=\sum_{f \in F} Y_{f} \frac{x_{f}^{2}+y_{f}^{2}}{2}
$$

with respect to $\omega^{\mathbb{C}^{F}}:=\frac{\mathrm{i}}{4 \pi} \sum_{f \in F} \mathrm{~d} z_{f} \wedge \mathrm{d} \bar{z}_{f}=\frac{1}{2 \pi} \sum_{f \in F} \mathrm{~d} x_{f} \wedge \mathrm{d} y_{f}$, if $z_{f}=x_{f}+\mathrm{i} y_{f}$, with $x_{f}, y_{f} \in \mathbb{R}$.

Since (6.2) depends linearly on $Y$, we view $\mu(z)$ as an element of $\left(\mathbb{R}^{F}\right)^{*} \simeq \mathbb{R}^{F}$ with coordinates

$$
\mu(z)_{f}=\left|z_{f}\right|^{2} / 2=\left(x_{f}^{2}+y_{f}^{2}\right) / 2, \quad f \in F .
$$

In other words, the action of $\mathbb{R}^{F} / \mathbb{Z}^{F}$ on $\mathbb{C}^{F}$ is Hamiltonian with respect to $\omega^{\mathbb{C}^{F}}$ and with momentum map $\mu: \mathbb{C}^{F} \rightarrow\left(\operatorname{Lie}\left(\mathbb{R}^{F} / \mathbb{Z}^{F}\right)\right)^{*}$ given by (6.3). It follows that the action of $N$ on $\mathbb{C}^{F}$ is Hamiltonian with momentum map $\mu_{N}:=\iota_{\mathfrak{n}}^{*} \circ \mu: \mathbb{C}^{F} \rightarrow \mathfrak{n}^{*}$, where $\iota_{\mathfrak{n}}: \mathfrak{n} \rightarrow \mathbb{R}^{F}$ denotes the identity and $\iota_{\mathfrak{n}}^{*}:\left(\mathbb{R}^{F}\right)^{*} \rightarrow \mathfrak{n}^{*}$ its transpose, which assigns to a linear form on $\mathbb{R}^{F}$ its restriction to $\mathfrak{n}$.

Let $\lambda$ denote the element of $\left(\mathbb{R}^{F}\right)^{*} \simeq \mathbb{R}^{F}$ with the coordinates $\lambda_{f}, f \in F$. Write $\lambda_{N}=\iota_{\mathfrak{n}}^{*}(\lambda)$. It follows from Guillemin [70, Theorems 1.6 and 1.4] that $\lambda_{N}$ is a regular value of $\mu_{N}$. Hence, $Z:=\mu_{N}^{-1}\left(\left\{\lambda_{N}\right\}\right)$ is a smooth submanifold of $\mathbb{C}^{F}$, and the action of the torus $N$ on $Z$ is free. By Theorem 4.1 the $N$-orbit space $M_{\Delta}:=Z / N$ is a smooth $2 n$-dimensional manifold such that the projection $p: Z \rightarrow M_{\Delta}$ exhibits $Z$ as a principal $N$-bundle over $M_{\Delta}$. Moreover, there is a unique symplectic form $\omega_{\Delta}$ on $M_{\Delta}$ such that $p^{*} \omega_{\Delta}=\iota_{Z} \omega^{\mathbb{C}^{F}}$, where $\iota_{Z}$ is the identity viewed as a smooth mapping from $Z$ to $\mathbb{C}^{F}\left(M_{\Delta}\right.$ is the Marsden-WeinsteinMeyer reduction of $\left.\left(\mathbb{C}^{F}, \omega^{\mathbb{C}^{F}}\right)\right)$.

On the $N$-orbit space $M_{\Delta}$, we still have the action of the torus $\left(\mathbb{R}^{F} / \mathbb{Z}^{F}\right) / N \simeq T$, with momentum mapping $\mu_{\Delta}: M \rightarrow \mathfrak{t}^{*}$ determined by $\pi^{*} \circ \mu_{\Delta} \circ p=\left.(\mu-\lambda)\right|_{Z}$. The torus $T$ acts effectively on $M$ and $\mu_{\Delta}(M)=\Delta$ (see Guillemin [70, Theorem 1.7]), and therefore we have constructed a symplectic-toric manifold $\left(M_{\Delta}, \omega_{\Delta}\right)$ with $T$ action and momentum map $\mu_{\Delta}: M_{\Delta} \rightarrow \mathfrak{t}^{*}$ such that $\mu_{\Delta}(M)=\Delta$.

The fiber over $t \in \Delta$ of the momentum map is diffeomorphic to a torus of dimension equal to the dimension of the lowest-dimensional face $F$ of $\Delta$ such that $t \in F$.

There have been generalizations of Theorem 6.4. for instance to multiplicity-free group actions by Woodward [165], and to symplectic-toric orbifolds by Lerman and Tolman [107]. An extension to noncompact symplectic manifolds has been recently given by Karshon and Lerman [90.

Because any symplectic-toric manifold is obtained by symplectic reduction of $\mathbb{C}^{F}$, it admits a compatible $T$-invariant Kähler metric. Delzant [33, Section 5] observed that $\Delta$ gives rise to a fan, and that the symplectic-toric manifold with Delzant polytope $\Delta$ is $T$-equivariantly diffeomorphic to the toric variety $M^{\text {toric }}$ defined by the fan. Here $M^{\text {toric }}$ is a complex $n$-dimensional complex analytic manifold, and the action of $T$ on $M^{\text {toric }}$ has an extension to a complex analytic action on $M^{\text {toric }}$ of the complexification $T_{\mathbb{C}}$ of $T$. 
A detailed study of the relation between the symplectic-toric manifold and $M^{\text {toric }}$ appears in 41]. The following proof illustrates the interplay between the symplectic and algebraic viewpoints.

Proposition 6.5. Every symplectic-toric manifold is simply connected.

Proof. A symplectic-toric manifold may be provided with the structure of a toric variety defined by a complete fan (Delzant [33, Section 5], Guillemin [70, Appendix 1]). Danilov [31, Theorem 9.1] observed that such a toric variety is simply connected: indeed, it has an open cell which is isomorphic to the complex space $\mathbb{C}^{n}$, whose complement is a complex subvariety of complex codimension 1, so all loops may be deformed into the cell and contracted within the cell to a point.

Remark 6.6. The manifold KT in (5.2) fits in the third case in Kodaira 96, Theorem 19]. Thurston rediscovered it in [152 and observed that there is no Kähler structure on KT compatible with the symplectic form (by noticing that the first Betti number $b_{1}(\mathrm{KT})$ is 3 ). It follows from Proposition 6.5 that no other symplectic 2 -torus action on KT is Hamiltonian.

6.2. Classification of log symplectic-toric manifolds. Recently there has been a generalization of symplectic-toric geometry to a class of Poisson manifolds, called log-symplectic manifolds.

Log-symplectic manifolds are generically symplectic, and they degenerate along a normal crossing configuration of smooth hypersurfaces. Guillemin, Miranda, Pires, and Scott initiated their study in [71. They proved the analogue of Delzant's theorem (Theorem 6.4) in the case where the degeneracy locus of the associated Poisson structure is a smooth hypersurface.

Most often degeneracy loci for Poisson structures are singular. In 69] the authors considered the mildest possible singularities, normal crossing hypersurfaces, and gave an analogue of Theorem 6.4. Next we informally state this result to give a flavor of the ingredients involved (being precise would be beyond the scope of this paper). The notion of isomorphism below generalizes the classical notion taking into account the log-symplectic structure.

Theorem $6.7([69])$. There is a one-to-one correspondence between isomorphism classes of oriented compact connected log symplectic-toric $2 n$-manifolds and equivalence classes of pairs $(\Delta, M)$, where $\Delta$ is a compact convex log affine polytope of dimension $n$ satisfying the Delzant condition and $M \rightarrow \Delta$ is a principal $n$-torus bundle over $\Delta$ with a vanishing toric log obstruction class.

Log-symplectic geometry and its toric version are an active area of research related to tropical geometry and the extended tropicalizations of toric varieties of Kajiwara [87] and Payne [125.

Convexity properties of Hamiltonian torus actions on log-symplectic manifolds were studied in [72, where the authors prove a generalization of Theorem 4.3 .

6.3. Classification of Hamiltonian $S^{1}$-spaces. In addition to Delzant's classification (Theorem 6.4) there have been other classifications of Hamiltonian $G$-actions on compact symplectic $2 n$-manifolds. In this section we outline the classification when $G=S^{1}$ and $n=2$, due to Karshon.

Definition 6.8. A Hamiltonian $S^{1}$-space is a compact connected symplectic 4manifold $(M, \omega)$ equipped with an effective Hamiltonian $S^{1}$-action. 
This definition is valid for manifolds of any dimension $2 n \geqslant 2$, but since only the two- and four-dimensional cases are completely understood (and the two-dimensional case is included in Delzant's theorem), the terminology Hamiltonian $S^{1}$-space is traditionally reserved for $2 n=4$.

Let $\left(M, \omega, S^{1}\right)$ be a Hamiltonian $S^{1}$-space. We assign to it a labeled graph as follows. Let $\mu: M \rightarrow \mathbb{R}$ be the momentum map of the $S^{1}$-action. For each component $\Sigma$ of the set of fixed points of the $S^{1}$-action there is one vertex in the graph, labeled by $\mu(\Sigma) \in \mathbb{R}$.

If $\Sigma$ is a surface, the corresponding vertex has two additional labels - one is the symplectic area of $\Sigma$ and the other one is the genus of $\Sigma$.

Let $F_{k}$ be a subgroup of $k$ elements of $S^{1}$. For every connected component $C$ of the set of points fixed by $F_{k}$, there is an edge in the graph, labeled by the integer $k>1$. The component $C$ is a 2 -sphere, which we call a $F_{k}$-sphere. The quotient circle $S^{1} / F_{k}$ rotates it while fixing two points, and the two vertices in the graph corresponding to the two fixed points are connected in the graph by the edge corresponding to $C$.

Theorem 6.9 (Ahara and Hattori 3, and Audin [12,13]). Every Hamiltonian $S^{1}$-space is $S^{1}$-equivariantly diffeomorphic to a complex surface with a holomorphic $S^{1}$-action which is obtained from $\mathbb{C} P^{2}$, a Hirzebruch surface, or a $\mathbb{C} P^{1}$-bundle over a Riemann surface with appropriate circle actions by a sequence of blowups at the fixed points.

Let $A$ and $B$ be connected components of the set of fixed points. The $S^{1}$-action extends to a holomorphic action of the group $\mathbb{C}^{\times}$of nonzero complex numbers. Consider the time flow given by the action of the subgroup $\{\exp (t) \mid t \in \mathbb{R}\}$.

Definition 6.10. The component $A$ is greater than the component $B$ if there is an orbit of the $\mathbb{C}^{\times}$-action which at time $t=\infty$ approaches a point in $A$, and at time $t=-\infty$, a point in $B$.

Take any of the complex surfaces with $S^{1}$-actions considered by Ahara and Hattori, and Audin, and assign a real parameter to every connected component of the fixed point set such that these parameters are monotonic with respect to the partial ordering we have just described. If the manifold contains two fixed surfaces, one assigns a positive real number to each of them so that the difference between the numbers is given by a formula involving the previously chosen parameters.

Karshon proved that for every such a choice of parameters there exists an invariant symplectic form and a momentum map on the complex surface such that the values of the momentum map at the fixed points and the symplectic areas of the fixed surfaces are equal to the chosen parameters. Moreover, every two symplectic forms with this property differ by an $S^{1}$-equivariant diffeomorphism.

Theorem 6.11 (Karshon 89]). If two Hamiltonian $S^{1}$-spaces have the same graph, then they are $S^{1}$-equivariantly symplectomorphic. Moreover, every compact fourdimensional Hamiltonian $S^{1}$-space is $S^{1}$-equivariantly symplectomorphic to one of the spaces listed in the paragraph above.

A generalization of this classification result to higher dimensions has been recently obtained by Karhson and Tolman [91. The authors construct all possible Hamiltonian torus actions for which all the nonempty reduced spaces are two dimensional (and not single points), the manifold is connected, and the momentum map is proper as a map to a convex set. 
The study of symplectic and Hamiltonian circle actions is an active topic of research; see for instance McDuff and Tolman [117. where they show many interesting properties. For instance, they prove that if the weights of a Hamiltonian $S^{1}$-action on a compact symplectic symplectic manifold $(M, \omega)$ at the points at which the momentum map is a maximum are sufficiently small, then the circle represents a nonzero element of $\pi_{1}(\operatorname{Ham}(M, \omega))$, where $\operatorname{Ham}(M, \omega)$ is the group of Hamiltonian symplectomorphisms of $(M, \omega)$. See also Sections 3.4 and 4.4 , where we discuss properties of symplectic $S^{1}$-actions that are not necessarily Hamiltonian. In this direction, see also 62,63 .

In 65. Godinho and Sabatini construct an algorithm to obtain linear relations among the weights at the fixed points which under certain conditions determine a family of vector spaces which contain the admissible lattices of weights.

6.4. Classification of symplectic-semitoric manifolds. Semitoric systems, or symplectic-semitoric manifolds, are a rich class of integrable systems which, in the case of compact manifolds, takes place on Karshon's Hamiltonian $S^{1}$-spaces. Let $(M, \omega)$ be a symplectic four-dimensional manifold. The Poisson brackets of $f, g \in \mathrm{C}^{\infty}(M)$ are defined by $\{f, g\}:=\omega\left(\mathcal{X}_{f}, \mathcal{X}_{g}\right)$, where $\mathcal{X}_{f}$ is as in (3.5).

Definition 6.12. An integrable on $(M, \omega)$ is a smooth map $F=\left(f_{1}, f_{2}\right): M \rightarrow \mathbb{R}^{2}$ such that $\left\{f_{1}, f_{2}\right\}=0$, and $\mathcal{X}_{f_{1}}, \mathcal{X}_{f_{2}}$ are linearly independent almost everywhere.

A general theorem of Eliasson characterizes the so-called nondegenerate singularities (the term "nondegenerate" here is a natural generalization of "Morse nondegenerate" which is more involved to define [138). The following is a particular instance of this general theorem and is of interest to us.

Theorem 6.13 (Eliasson 47,48). Let $F:=\left(f_{1}, f_{2}\right):(M, \omega) \rightarrow \mathbb{R}^{2}$ be an integrable system, all of the singularities of which are nondegenerate, and with no hyperbolic blocks. Then there exist local symplectic coordinates $\left(x_{1}, x_{2}, \xi_{1}, \xi_{2}\right)$ about every nondegenerate critical point $m$, in which $m=(0,0,0,0)$, and

$$
(F-F(m)) \circ \varphi=g \circ\left(q_{1}, q_{2}\right),
$$

where $\varphi=\left(x_{1}, x_{2}, \xi_{1}, \xi_{2}\right)^{-1}$ and $g$ is a diffeomorphism from a small neighborhood of the origin in $\mathbb{R}^{4}$ into another such neighborhood, such that $g(0,0,0,0)=(0,0,0,0)$ and $\left(q_{1}, q_{2}\right)$ is, depending on the rank of the critical point, as follows. If $m$ is a critical point of $F$ of rank $0,\left(q_{1}, q_{2}\right)$ is given by $q_{1}=\left(x_{1}^{2}+\xi_{1}^{2}\right) / 2$ and $q_{2}=\left(x_{2}^{2}+\xi_{2}^{2}\right) / 2$, or

$$
q_{1}=x_{1} \xi_{2}-x_{2} \xi_{1} \text { and } q_{2}=x_{1} \xi_{1}+x_{2} \xi_{2} .
$$

If $m$ is a critical point of $F$ of rank 1 , then $q_{1}=\left(x_{1}^{2}+\xi_{1}^{2}\right) / 2$ and $q_{2}=\xi_{2}$.

The assumption of not having hyperbolic blocks is simply to reduce the complexity of the statement of the theorem, but it is not really needed to understand the discussion which follows.

Remark 6.14. The analytic case of Theorem 6.13 is due to Rüssmann [146] for two degrees of freedom systems, and to Vey [156] in any dimension.

Definition 6.15. A semitoric system $F:=\left(f_{1}, f_{2}\right): M \rightarrow \mathbb{R}^{2}$ on a connected symplectic 4-manifold $(M, \omega)$ is an integrable system such that $f_{1}$ is the momentum map of an effective Hamiltonian $S^{1}$-action, $f_{1}$ is a proper map, and the singularities 
of $F$ are nondegenerate, without hyperbolic blocks, and hence they are of the form given in Theorem 6.13

Remark 6.16. Above, $f_{1}$ gives rise to a Hamiltonian $S^{1}$-action on $M$, and $f_{2}$ gives rise to a Hamiltonian $\mathbb{R}$-action on $M$. Their flows, one after another, define a Hamiltonian $\left(S^{1} \times \mathbb{R}\right)$-action. The precise relation between $\left(S^{1} \times \mathbb{R}\right)$-actions and semitoric systems appears in [51, Section 3].

Definition 6.17. Let $F:=\left(f_{1}, f_{2}\right):(M, \omega)$ and $F^{\prime}:=\left(f_{1}^{\prime}, f_{2}^{\prime}\right):\left(M^{\prime}, \omega^{\prime}\right) \rightarrow \mathbb{R}^{2}$ be semitoric systems. They are isomorphic if there exists a symplectomorphism $\varphi:(M, \omega) \rightarrow\left(M^{\prime}, \omega^{\prime}\right)$ and a smooth map $\phi: F(M) \rightarrow \mathbb{R}$ with $\partial_{2} \phi>0$, such that $\varphi^{*} f_{1}^{\prime}=f_{1}$ and $\varphi^{*} f_{2}^{\prime}=\phi\left(f_{1}, f_{2}\right)$.

Semitoric systems are classified if each singular fiber contains at most one singular point of type (6.4); these are called focus-focus (or nodal, in algebraic geometry). The singular fiber containing a focus-focus point is a 2-torus pinched at the focusfocus point (i.e., topologically a 2 -sphere with the north and south poles identified); systems satisfying this condition are called simple.

Theorem 6.18 (136, 137). Simple semitoric systems $F:=\left(f_{1}, f_{2}\right):(M, \omega) \rightarrow$ $\mathbb{R}^{2}$ are determined, up to isomorphisms, by a convex polygon $\Delta$ endowed with a finite collection of interior points, each of which is labeled by a tuple $(k \in$ $\left.\mathbb{Z}, \sum_{i, j=1}^{\infty} a_{i j} X^{i} Y^{j}\right)$. Here $\Delta$ is obtained from $F(M)$ by appropriately unfolding the singular affine structure induced by $F$, the interior points in $\Delta$ are the images of the focus-focus values under the map $F(M) \rightarrow \Delta$ which unfolds the singular affine structure, $k$ encodes how twisted the singular Lagrangian fibration $F$ is at the focus-focus point, and the Taylor series $\sum_{i, j=1}^{\infty} a_{i j} X^{i} Y^{j}$ encodes the singular dynamics of the vector fields $\mathcal{X}_{f_{1}}, \mathcal{X}_{f_{2}}$ at the particular focus-focus point. Conversely, given a convex polygon 1 with interior points $p_{1}, \ldots, p_{n}$, and for each $p_{\ell}$ a label $\left(k \in \mathbb{Z}, \sum_{i, j=1}^{\infty} a_{i j} X^{i} Y^{j}\right)$, one can construct $(M, \omega)$ and a semitoric system $F:(M, \omega) \rightarrow \mathbb{R}^{2}$ having this data as invariants.

In 123. Palmer defined the moduli space of semitoric systems, which is an incomplete metric space, and constructed its completion. In 88] the connectivity properties of this space were studied.

Four-dimensional symplectic-toric manifolds (treated in Section 6.1.2) are a particular case of compact semitoric systems (the only invariant is the convex polygon). Every semitoric system takes place on a Hamiltonian $S^{1}$-space, and the relation has been made explicit recently. We call a Karshon graph the labeled directed graph in Theorem 6.11.

Theorem 6.19 (Hohloch, Sabatini, and Sepe 84]). Let $F:=\left(f_{1}, f_{2}\right):(M, \omega) \rightarrow \mathbb{R}^{2}$ be a simple semitoric system on a compact manifold with $n$ focus-focus critical points and an underlying Hamiltonian $S^{1}$-space $\left(M, \omega, S^{1}\right)$ with momentum map $f_{1}$. Then the convex polygon $\Delta$ in Theorem 6.18 and $n$ determine the Karshon graph, thus classifying $\left(M, \omega, S^{1}\right)$ up to $S^{1}$-equivariant symplectomorphisms.

There has been recent work generalizing the convex polygon in Theorem 6.18 to higher-dimensional semitoric systems by Wacheux [157. The Fomenko school

\footnotetext{
${ }^{1}$ This is really not just any polygon, but a polygon of so-called semitoric type, which generalizes the notion of a Delzant polygon (which was applicable to symplectic-toric manifolds) to this more general context.
} 
has powerful and far-reaching methods to study the topology of singularities of integrable systems [21]. In [140, Section 7] there is an outline of how to carry out the theory of symplectic-toric manifolds and integrable systems we have just described in the case when $\mathbb{R}$ is replaced by the $p$-adic numbers (in the context of the univalent formalization of the $p$-adic numbers).

Remark 6.20. In general, symplectic $G$-capacities (Section 3.5) provide a general setting to define monotonic invariants of integrable systems.

\section{Properties of Symplectic actions}

This section summarizes basic properties of general symplectic actions, including, under certain restrictions (of what types of orbits are allowed), a precise description of their stabilizer subgroups and symplectic normal forms in a neighborhood of an orbit. These are required ingredients for the construction of symplectic invariants in the upcoming section but they are also of independent interest.

7.1. Fundamental form of a symplectic action. Let $T$ be a torus with Lie algebra $\boldsymbol{t}$. Suppose that $T$ acts symplectically on a connected symplectic manifold $(M, \omega)$.

Proposition 7.1. There is a unique antisymmetric bilinear form $\omega^{\mathfrak{t}}$ on $\mathfrak{t}$ such that

$$
\omega^{\mathfrak{t}}(X, Y)=\omega_{x}\left(X_{M}(x), Y_{M}(x)\right)
$$

for every $X, Y \in \mathfrak{t}$ and every $x \in M$. It is called the fundamental form.

Proof. Let $\mathcal{X}$ and $\mathcal{Y}$ be smooth vector fields on $M$ satisfying $\mathrm{L}_{\mathcal{X}} \omega=0$ and $\mathrm{L}_{\mathcal{Y}} \omega=0$. Then, since $\mathrm{L}_{\mathcal{X}} \omega=0$, by the homotopy identity (2.3) and the closedness of $\omega$,

$$
\mathrm{i}_{[\mathcal{X}, \mathcal{Y}]} \omega=\mathrm{L}_{\mathcal{X}}\left(\mathrm{i}_{\mathcal{Y}} \omega\right)=\mathrm{i}_{\mathcal{X}}\left(\mathrm{d}\left(\mathrm{i}_{\mathcal{Y}} \omega\right)\right)+\mathrm{d}\left(\mathrm{i}_{\mathcal{X}}\left(\mathrm{i}_{\mathcal{Y}} \omega\right)\right)=-\mathrm{d}(\omega(\mathcal{X}, \mathcal{Y})) .
$$

Take $\mathcal{X}=X_{M}, \mathcal{Y}=Y_{M}, X, Y \in \mathfrak{t}$. The Lie brackets of two vector fields vanish when the flows of the vector fields commute, so $\left[X_{M}, Y_{M}\right]=0$. Then, by (7.2), $\mathrm{d}\left(\omega\left(X_{M}, Y_{M}\right)\right)=0$, and the connectedness of $M$ implies that $x \mapsto$ $\omega_{x}\left(X_{M}(x), Y_{M}(x)\right)$ is constant.

The fundamental form $\omega^{t}$ is an essential ingredient in the study of symplectic actions. In the case of Hamiltonian actions, it takes a simple form: if $(M, \omega)$ is a compact connected symplectic $2 n$-manifold endowed with an effective symplectic action of an $n$-torus $T$, the $T$-action is Hamiltonian if and only if $\omega^{\mathrm{t}}=0$ and $\mathrm{H}^{1}(M / T ; \mathbb{R})=0$ (compare with Theorem 3.15).

7.2. Benoist-Ortega-Ratiu symplectic normal form. We state the main result of this section for a general proper Lie group action (see Section 3.1 for the notion of proper action).

Let $(M, \omega)$ be a symplectic manifold with a proper symplectic action of a Lie group $G$. Let $x \in M, H:=G_{x}$. Let $\mathfrak{l}$ be the kernel of $\omega^{\mathfrak{t}}, \mathfrak{g}_{M}(x):=\mathrm{T}_{x}(G \cdot x)$, and let $\alpha_{x}$ be as in Proposition 3.2

Let $\omega^{G / H}$ be the $G$-invariant closed 2-form $\left(\alpha_{x}\right)^{*} \omega$ on $G / H$, and let $\omega^{W}$ be the symplectic form on $W:=\mathfrak{g}_{M}(x)^{\omega_{x}} /\left(\mathfrak{g}_{M}(x)^{\omega_{x}} \cap \mathfrak{g}_{M}(x)\right)$ defined as the restriction to $\mathfrak{g}_{M}(x)^{\omega_{x}}$ of $\omega_{x}$.

The map $X+\mathfrak{h} \mapsto X_{M}(x)$ is a linear isomorphism from $\mathfrak{l} / \mathfrak{h}$ to $\mathfrak{g}_{M}(x)^{\omega_{x}} \cap \mathfrak{g}_{M}(x)$. The linearized action of $H$ on $\mathrm{T}_{x} M$ is symplectic and leaves $\mathfrak{g}_{M}(x) \simeq \mathfrak{g} / \mathfrak{h}$ invariant, acting on it via the adjoint representation. It also leaves $\mathfrak{g}_{M}(x)^{\omega_{x}}$ invariant and 
induces an action of $H$ on $\left(W, \omega^{W}\right)$ by symplectic linear transformations. Let $E:=(\mathfrak{l} / \mathfrak{h})^{*} \times W$, on which $h \in H$ acts by sending $(\lambda, w)$ to $\left(\left((\operatorname{Ad}(h))^{*}\right)^{-1}(\lambda), h \cdot w\right)$. Choose $\mathrm{Ad} H$-invariant linear complements $\mathfrak{k}$ and $\mathfrak{c}$ of $\mathfrak{h}$ and $\mathfrak{l}$ in $\mathfrak{g}$, respectively. Let $X \mapsto X_{\mathfrak{l}}: \mathfrak{g} \rightarrow \mathfrak{l}$ and $X \mapsto X_{\mathfrak{h}}: \mathfrak{g} \rightarrow \mathfrak{h}$ denote the linear projection from $\mathfrak{g}$ onto $\mathfrak{l}$ and $\mathfrak{h}$ with kernel equal to $\mathfrak{c}$ and $\mathfrak{k}$, respectively. These projections are $\operatorname{Ad} H$-equivariant.

Define the 1-form $\eta^{\#}$ on $G \times E$ by

$$
\eta_{(g,(\lambda, w))}^{\#}\left(\left(\mathrm{~d}_{1} \mathrm{~L}_{g}\right)(X),(\delta \lambda, \delta w)\right):=\lambda\left(X_{\mathfrak{l}}\right)+\frac{1}{2} \omega^{W}\left(w, \delta w+X_{\mathfrak{h}} \cdot w\right)
$$

for all $g \in G, \lambda \in(\mathfrak{l} / \mathfrak{h})^{*}, w \in W$, and all respective vectors $X \in \mathfrak{g}, \delta \lambda \in(\mathfrak{l} / \mathfrak{h})^{*}$, $\delta w \in W$.

Let $G \times{ }_{H} E$ denote the orbit space of $G \times E$ for the proper and free action of $H$ on $G \times E$, where $h \in H$ acts on $G \times E$ by sending $(g, e)$ to $\left(g h^{-1}, h \cdot e\right)$.

The action of $G$ on $G \times{ }_{H} E$ is induced by the translational action of $G$ on $G \times E$.

Let $\pi: G \times{ }_{H} E \rightarrow G / H$ be induced by $(g, e) \mapsto g: G \times E \rightarrow G$. Because $H$ acts on $E$ by means of linear transformations, this projection exhibits $G \times{ }_{H} E$ as a $G$-homogeneous vector bundle over the homogeneous space $G / H$, with fiber $E$ and structure group $H$.

If $\pi_{H}: G \times E \rightarrow G \times{ }_{H} E$ denotes $H$-orbit mapping, then there is a unique smooth 1-form $\eta$ on $G \times{ }_{H} E$, such that $\eta^{\#}=\pi_{H}{ }^{*} \eta$.

Endow $G \times{ }_{H} E$ with the 2-form $\pi^{*} \omega^{G / H}+\mathrm{d} \eta$. This 2-form is symplectic.

The following is the local normal form of Benoist [15, Proposition 1.9] and Ortega and Ratiu 120] for a general proper symplectic Lie group action.

Theorem 7.2 (Benoist [15, Ortega and Ratiu 120]). There is an open H-invariant neighborhood $E_{0}$ of the origin in $E$, an open $G$-invariant neighborhood $U$ of $x$ in $M$, and a G-equivariant symplectomorphism $\Phi:\left(G \times{ }_{H} E, \pi^{*} \omega^{G / H}+\mathrm{d} \eta\right) \rightarrow(U, \omega)$ such that $\Phi(H \cdot(1,0))=x$.

For Hamiltonian actions this result had been obtained before by Marle [109] and Guillemin and Sternberg [76, Section 41].

7.3. Symplectic, Lagrangian, or coisotropic orbits. Later in the paper we will give classifications of classes of symplectic actions, depending on what type of orbits they have - symplectic, Lagrangian, or coisotropic.

7.3.1. Symplectic orbits. The first general type of symplectic actions we are interested in is the following.

Definition 7.3. Suppose that a torus $T$ acts effectively and symplectically on a compact connected symplectic manifold. If there is a symplectic $T$-orbit of the largest dimension, that is, a $\operatorname{dim} T$-dimensional symplectic orbit, we say that the $T$-action is a maximal symplectic action.

Examples satisfying this definition were given in Section 5.2.1

Remark 7.4. Since symplectic manifolds are even dimensional (Proposition 2.8), $\operatorname{dim} T$ is even.

Since the orbits of a Hamiltonian action are isotropic, we have the following:

Proposition 7.5. A maximal symplectic action is not Hamiltonian. 
7.3.2. Coisotropic orbits. Symplectic torus actions with some Lagrangian orbit fall in the category of coisotropic actions.

Definition 7.6. Suppose that a torus $T$ acts effectively and symplectically on a compact connected symplectic manifold. If there is a coisotropic $T$-orbit, we call the $T$-action coisotropic.

The simplest coisotropic actions are given in Examples 5.1 and 5.2 (Figure 1); in these examples the coisotropic action is also Hamiltonian (in contrast with Proposition (7.5). In fact, the principal orbits of a symplectic-toric manifold are Lagrangian, and hence coisotropic, so every manifold in Delzant's classification (Theorem 6.4) gives an example of a coisotropic action. We gave other examples in Section 5.2 .2 .

The following is a consequence of Theorem 7.2

Corollary 7.7. Let $(M, \omega)$ be a compact connected symplectic manifold, and let $T$ be a torus which acts effectively and symplectically on $(M, \omega)$. If $x \in M$ and the orbit $T \cdot x$ is coisotropic, then $T \cdot x$ is a principal orbit. Furthermore, $\operatorname{dim} M=$ $\operatorname{dim} T+\operatorname{dim} \mathfrak{l}$.

Proof. We use Theorem 7.2 with $G=T$. Since $T$ is Abelian, the adjoint action of $H=T_{x}$ on $\mathfrak{t}$ is trivial, which implies that the coadjoint action of $H$ on the component $(\mathfrak{l} / \mathfrak{h})^{*}$ is trivial. Let $T \cdot x$ be a coisotropic orbit. Then $W$ is trivial. This implies that the action of $H$ on $E=(\mathfrak{l} / \mathfrak{h})^{*}$ is trivial, and $T \times_{H} E=T \times_{H}(\mathfrak{l} / \mathfrak{h})^{*}$ is $T$-equivariantly isomorphic to $(T / H) \times(\mathfrak{l} / \mathfrak{h})^{*}$, where $T$ acts by left multiplication on the first factor. It follows that in the model all stabilizer subgroups are equal to $H$, and therefore $T_{y}=H$ for all $y$ in the $T$-invariant open neighborhood $U$ of $x$ in $M$. Since $M_{\text {reg }}$ is dense in $M$, there are $y \in U$ such that $T_{y}=\{1\}$, and therefore $T_{x}=H=\{1\}$, so the orbit $T \cdot x$ is principal. The statement $\operatorname{dim} M=\operatorname{dim} T+\operatorname{dim} \mathfrak{l}$ also follows.

A similar argument to that in the proof of Corollary 7.7 using Theorem 7.2 shows:

Proposition 7.8. Let $(M, \omega)$ be a compact connected symplectic manifold, and let $T$ be a torus which acts effectively and symplectically on $(M, \omega)$. If there exists a coisotropic principal T-orbit, then all principal T-orbits are coisotropic.

In a similar vein, one can show that every principal $T$-orbit is Lagrangian if and only if some principal $T$-orbit is Lagrangian, if and only if $\operatorname{dim} M=2 \operatorname{dim} T$ and $\omega^{\mathrm{t}}=0$.

In Guillemin and Sternberg [77] we find the following notion.

Definition 7.9. A symplectic manifold with a Hamiltonian action of a compact Lie group is called a multiplicity-free space if the Poisson brackets of any pair of invariant smooth functions vanish.

Proposition 7.10. For a torus $T$ acting on a compact connected symplectic manifold $(M, \omega)$, the principal orbits are coisotropic if and only if $(M, \omega)$ is a multiplicityfree space.

Proof. Let $x \in M_{\mathrm{reg}}$, and let $\mathfrak{t}_{M}(x):=\mathrm{T}_{x}(T \cdot x)$. Since $M_{\mathrm{reg}}$ is fibered by the $T$-orbits, $\mathfrak{t}_{M}(x)$ is the common kernel of the $\mathrm{d} f(x)$, with $f$ any $T$-invariant smooth function. Because $-\mathrm{d} f=\mathrm{i}_{\mathcal{X}_{f}} \omega$, we have that $\mathfrak{t}_{M}(x)^{\omega_{x}}$ is the set of all $\mathcal{X}_{f}(x)$, with $f$ any $T$-invariant smooth function. So if the principal $T$-orbits are coisotropic, 
$\mathcal{X}_{f}(x), \mathcal{X}_{g}(x) \in \mathfrak{t}_{M}(x)^{\omega_{x}} \cap \mathfrak{t}_{M}(x)$ for any $T$-invariant functions $f, g$. It follows that $\{f, g\}(x)=\omega_{x}\left(\mathcal{X}_{f}(x), \mathcal{X}_{g}(x)\right)=0$. Since $M_{\text {reg }}$ is dense in $M,\{f, g\} \equiv 0$. The converse is similar.

7.4. Stabilizer subgroups. Let $T$ be a torus, and let $(M, \omega)$ be a compact connected symplectic manifold endowed with an effective symplectic action of $T$. If $x \in M$, when is the stabilizer subgroup $T_{x}$ of $x$ connected? We know from Proposition 3.1 that $T_{x}$ is a Lie subgroup of $T$ and by Proposition 3.2 that the quotient $T / T_{x}$ is diffeomorphic to $T \cdot x$, so understanding $T_{x}$ helps us to understand $T \cdot x$. Moreover, $T_{x}$ is essential in the symplectic normal form (Section 7.2), which we use later.

Let $\mathfrak{t}_{x}$ be the Lie algebra of $T_{x}$, which consists of the $X \in \mathfrak{t}$ such that $X_{M}(x)=0$. That is, $\mathfrak{t}_{x}$ is the kernel of the map $\alpha_{x}: X \mapsto X_{M}(x)$ from $\mathfrak{t}$ to $\mathrm{T}_{x} M$.

Lemma $7.11([128])$. Let $(M, \omega)$ be a compact connected symplectic manifold with a symplectic $T$-action. If the $T$-action is maximal symplectic, then $T_{x}$ is a finite Abelian group for every $x \in M$.

Proof. Since the fundamental form $\omega^{t}$ is point-independent, it is nondegenerate and $\operatorname{ker} \omega^{\mathfrak{t}}=0$. Since $\mathfrak{t}_{x} \subset \operatorname{ker} \omega^{\mathfrak{t}}, \mathfrak{t}_{x}$ is trivial.

Corollary 7.12. Let $(M, \omega)$ be a symplectic manifold endowed with an effective symplectic $T$-action. If there is a symplectic $\operatorname{dim} T$-orbit, then every $T$-orbit is symplectic and $\operatorname{dim} T$-dimensional.

Proof. By Lemma 7.11 $\operatorname{dim} T_{x}=0$. The result follows from the existence of the diffeomorphism $T / T_{x} \rightarrow T \cdot x$ in Proposition 3.2 .

Since every $T_{x}$ is finite, it follows from the tube theorem of Koszul (see [100] or [39. Theorem 2.4.1]) and the compactness of $M$ that there exists only finitely many different stabilizer subgroups.

The following is statement (1) (a) in [15, Lemma 6.7]:

Lemma 7.13 (Benoist [15]). Let $(M, \omega)$ be a compact connected symplectic manifold with a symplectic $T$-action. If the $T$-action is coisotropic, then $T_{x}$ is a subtorus of $T$.

Proof. We use Theorem 7.2 with $G:=T$, with $H:=T_{x}$ acting trivially on $(\mathfrak{l} / \mathfrak{h})^{*}$ in $E=(\mathfrak{l} / \mathfrak{h})^{*} \times W$. Recall that $t \in T$ acts on $T \times{ }_{H} E$ by sending $H \cdot\left(t^{\prime}, e\right)$ to $H \cdot\left(t t^{\prime}, e\right)$. When $t=h \in H, H \cdot\left(h t^{\prime}, e\right)=H \cdot\left(h t^{\prime} h^{-1}, h \cdot e\right)=H \cdot\left(t^{\prime}, h \cdot e\right)$ since $T$ is Abelian, and the action of $H$ on $T \times_{H} E$ is represented by the linear symplectic action of $H$ on $W$.

Since $\operatorname{dim} M=(\operatorname{dim} T+\operatorname{dim}(\mathfrak{l} / \mathfrak{h})+\operatorname{dim} W)-\operatorname{dim} H$ and because the assumption that the principal orbits are coisotropic implies $\operatorname{dim} M=\operatorname{dim} T+\operatorname{dim} \mathfrak{l}$ (see Corollary (7.7), $\operatorname{dim} W=2 \operatorname{dim} H$.

Write $m=\operatorname{dim} H$. The action of $H$ by means of symplectic linear transformations on $\left(W, \omega^{W}\right)$ leads to a direct sum decomposition of $W$ into pairwise $\omega^{W}$-orthogonal two-dimensional $H$-invariant linear subspaces $E_{j}, 1 \leqslant j \leqslant m$. For $h \in H$ and $1 \leqslant j \leqslant m$, let $\iota_{j}(h)$ be the restriction to $E_{j} \subset W \simeq\{0\} \times W \subset$ $(\mathfrak{l} / \mathfrak{h})^{*} \times W$ of the action of $h$ on $E$. Note that $\operatorname{det} \iota_{j}(h)=1$, because $\iota_{j}(h)$ preserves the restriction to $E_{j} \times E_{j}$ of $\omega^{W}$, which is an area form on $E_{j}$.

Averaging any inner product in each $E_{j}$ over $H$, we obtain an $H$-invariant inner product $\beta_{j}$ on $E_{j}$, and $\iota_{j}$ is a Lie group homomorphism from $H$ to $\mathrm{SO}\left(E_{j}, \beta_{j}\right)$, the 
group of linear transformations of $E_{j}$ which preserve $\beta_{j}$ and orientation. If $h \in$ $H, w \in W_{\text {reg }}$, then $h \cdot w=\sum_{j=1}^{m} \iota_{j}(h) w_{j}$ where $w=\sum_{j=1}^{m} w_{j}, w_{j} \in E_{j}$. Therefore $\iota_{j}(h) w_{j}=w_{j}$ for all $1 \leqslant j \leqslant m$ implies $h=1$. Hence $\iota: H \rightarrow \prod_{j=1}^{m} \operatorname{SO}\left(E_{j}, \beta_{j}\right)$, $i(h)=\left(\iota_{1}(h), \ldots, \iota_{m}(h)\right)$, is a Lie group isomorphism, so $H$ is connected. Hence $H$ is a subtorus of $T$.

\section{Classifications of symplectic actions}

The properties we have studied in Section 7 are going to allow us to construct symplectic invariants of general symplectic actions. The current constructions rely on the use of symplectic normal forms in the neighborhood of an orbit, and then use these normal forms to build a global model of the manifold, the action, and the symplectic form. In a few cases, there are enough constraints that it is possible to give complete classifications in the spirit of Delzant's pioneering work (Theorem 6.4) for symplectic Hamiltonian actions.

8.1. Classification of maximal symplectic actions. In this section we describe the invariants of a compact connected symplectic manifold $(M, \omega)$ endowed with a maximal symplectic $T$-action of a torus $T$ (as in Definition 7.3) and we use them to construct a model of $(M, \omega)$ and the $T$-action, up to $T$-equivariant symplectomorphisms. By Remark 7.4, $\operatorname{dim} M-\operatorname{dim} T$ is even, say $2 k$. Finally, we state a classification result, also up to $T$-equivariant symplectomorphisms, when $\operatorname{dim} T=\operatorname{dim} M-2$ (the "large" acting torus forces enough symmetries to be able to classify).

8.1.1. Orbit space. Let $\pi: M \rightarrow M / T$ be $\pi(x):=T \cdot x$. The orbit space $M / T$ is endowed with the maximal topology for which $\pi$ is continuous, which is a Hausdorff topology. Since $M$ is compact and connected, $M / T$ is compact and connected. By Lemma 7.11 $T_{x}$ is a finite Abelian group for every $x \in M$. By the tube theorem ([39, Theorem 2.4.1]) there is a $T$-invariant open neighborhood $U_{x}$ of $T \cdot x$, a disk $D_{x}$ disk-centered at $0 \in \mathbb{C}^{k}$, and a $T$-equivariant diffeomorphism $\Phi_{x}: U_{x} \rightarrow T \times_{T_{x}} D_{x}$, where the quotient on the right-hand side is obtained by the action of $h \in T_{x}$ on $T \times D_{x}$ which sends $(g, x)$ to $\left(g h^{-1}, h \cdot x\right), T_{x}$ acts by linear transformations on $D_{x}$, and the $T$-action on $T \times_{T_{x}} D_{x}$ is induced by the translational action of $T$ on the left factor of $T \times D_{x}$. Then $\Phi_{x}$ induces a homeomorphism $D_{x} / T_{x} \rightarrow \pi\left(U_{x}\right)$, which, when composed with the projection $D_{x} \rightarrow D_{x} / T_{x}$, gives a map $\phi_{x}: D_{x} \rightarrow \pi\left(U_{x}\right)$. It is easy to check that the collection $\left\{\left(\pi\left(U_{x}\right), D_{x}, \phi_{x}, T_{x}\right)\right\}_{x \in M}$ is an orbifold atlas for $M / T$. In the particular case that the symplectic $T$-action on $M$ is free (i.e., $T_{x}$ is trivial for every $x \in M), M / T$ is a smooth manifold, and this atlas is a smooth manifold atlas for $M / T$.

8.1.2. Flat connection. Consider the symplectic form $\omega^{\mathbb{C}^{k}}:=\frac{1}{2 \mathrm{i}} \sum_{j=1}^{k} \mathrm{~d} \overline{z^{j}} \wedge \mathrm{d} z^{j}$ on $\mathbb{C}^{k}$. The translational action of $T$ on $T \times \mathbb{C}^{k}$ descends to an action on $T \times{ }_{T_{x}} \mathbb{C}^{k}$. The fundamental form $\omega^{t}$ is nondegenerate, so it determines a unique symplectic form on $T$. The product symplectic form on $T \times \mathbb{C}^{k}$ is defined at $(t, z)$ and a pair of vectors $\left((X, u),\left(X^{\prime}, u^{\prime}\right)\right)$ by $\omega^{\mathfrak{t}}\left(X, X^{\prime}\right)+\omega^{\mathbb{C}^{k}}\left(u, u^{\prime}\right)$, and it descends to a symplectic form on $T \times_{T_{x}} E_{x}$, which we denote by $\omega^{\mathfrak{t}} \oplus \omega^{\mathbb{C}^{k}}$. Theorem 7.2 implies that for each $x \in M$ there exists an open $\mathbb{T}^{k}$-invariant neighborhood $E$ of 0 in $\mathbb{C}^{k}$, an open $T$-invariant neighborhood $V_{x}$ of $x$ in $M$, and a $T$-equivariant symplectomorphism 
$F_{x}:\left(T \times_{T_{x}} E, \omega^{\mathfrak{t}} \oplus \omega^{\mathbb{C}^{k}}\right) \rightarrow\left(V_{x}, \omega\right)$ such that $F_{x}\left([1,0]_{T_{x}}\right)=x$. It follows from this symplectic normal form that:

Proposition 8.1. The collection $\Omega:=\left\{\Omega_{x}\right\}_{x \in M}$, where $\Omega_{x}:=\left(\mathrm{T}_{x}(T \cdot x)\right)^{\omega_{x}}$, is a smooth distribution on $M$ and $\pi: M \rightarrow M / T$ is a smooth principal T-bundle for which $\Omega$ is a $T$-invariant flat connection.

Let $\psi: \widetilde{M / T} \rightarrow M / T$ be the universal cover of $M / T$ based at $p_{0}=\pi\left(x_{0}\right)$, $x_{0} \in M$. Let $\mathcal{I}_{x}$ be the maximal integral manifold of $\Omega$ containing $x \in M$. The inclusion $i_{x}: \mathcal{I}_{x} \rightarrow M$ is an injective immersion, and $\pi \circ i_{x}: \mathcal{I}_{x} \rightarrow M / T$ is an orbifold covering. Since $\widetilde{M / T}$ covers any covering of $M / T$, it covers $\mathcal{I}_{x}$, which is a manifold. Because a covering of a smooth manifold is a smooth manifold, $\widetilde{M / T}$ is a smooth manifold. Readers unfamiliar with orbifolds may consult [128, Section 9].

8.1.3. Monodromy. Let $\pi_{1}^{\text {orb }}(M / T)$ be the orbifold fundamental group of $M / T$ based at $p_{0}$. Then $\pi_{1}^{\text {orb }}(M / T) \times \widetilde{M / T} \rightarrow \widetilde{M / T},([\gamma],[\rho]) \mapsto[\rho \gamma]$, is a smooth action of $\pi_{1}^{\text {orb }}(M / T)$ on $\widetilde{M / T}$, which is transitive on each fiber $\widetilde{M / T}$ of $\psi: \widetilde{M / T} \rightarrow M / T$.

If $\rho:[0,1] \rightarrow M / T$ is a path with $\rho(0)=p_{0}$, let $\lambda_{\rho}:[0,1] \rightarrow M$ be the horizontal lift with respect to $\Omega$ such that $\lambda_{\rho}(0)=x_{0}$, where horizontal means $\mathrm{d} \lambda_{\rho}(t) / \mathrm{d} t \in$ $\Omega_{\lambda_{\rho(t)}}$ for every $t \in[0,1]$. Since the orbifold connection $\Omega$ is flat, $\lambda_{\rho}(1)=\lambda_{\delta}(1)$ if $\rho$ is homotopy equivalent to $\delta$ in the space of orbifold paths in $M / T$ which start at $p_{0}$ and end at a given point $p$. Therefore, there exists a unique group homomorphism $\mu: \pi_{1}^{\text {orb }}(M / T) \rightarrow T$ such that

$$
\lambda_{\gamma}(1)=\mu([\gamma]) \cdot x_{0}
$$

The homomorphism $\mu$ is the monodromy homomorphism of $\Omega$, and it does not depend on $x_{0} \in M$.

8.1.4. Model. Let $\pi_{1}^{\text {orb }}(M / T)$ act on $\widetilde{M / T} \times T$ by $[\gamma]([\rho], t)=\left(\left[\rho \gamma^{-1}\right], \mu([\gamma]) \cdot t\right)$. One can show that this action is free, and hence $\widetilde{M / T} \times{ }_{\pi_{1}^{\text {orb }}(M / T)} T$ is a compact connected smooth manifold. The $T$-action on $\widetilde{M / T} \times \times_{\pi_{1}^{\text {orb }}(M / T)} T$ is inherited from the translational $T$-action on the right factor of $\widetilde{M / T} \times T$. There is a unique symplectic form $\nu$ on $M / T$ such that $\left.\pi^{*} \nu\right|_{\Omega_{x}}=\left.\omega\right|_{\Omega_{x}}$ for every $x \in M$. The symplectic form on $\widetilde{M / T}$ is the pullback by $\widetilde{M / T} \rightarrow M / T$ of $\nu$, and the symplectic form on $T$ is the unique $T$-invariant symplectic form determined by $\omega^{\mathrm{t}}$. The symplectic form on $\widetilde{M / T} \times T$ is the product symplectic form, and it is inherited by $\widetilde{M / T} \times_{\pi_{1}^{\text {orb }}(M / T)} T$.

Theorem 8.2 ([128]). Let $(M, \omega)$ be a compact connected symplectic manifold endowed with a maximal symplectic $T$-action. Then $M$ is $T$-equivariantly symplectomorphic to $\widetilde{M / T} \times \pi_{\pi_{1}^{\text {orb }}(M / T)} T$.

Proof. For any $[\rho] \in \widetilde{M / T}$ and $t \in T$, define $\Phi([\rho], t):=t \cdot \lambda_{\rho}(1) \in M$. The assignment $([\rho], t) \mapsto \Phi([\rho], t)$ is a smooth covering $\Phi: \widetilde{M / T} \times T \rightarrow M$. By the definitions of the symplectic form and $T$-action, $\Phi$ induces a $T$-equivariant symplectomorphism from $\widehat{M / T} \times{ }_{\pi_{1}^{\text {orb }}\left(M / T, p_{0}\right)} T$ onto $M$. 
8.1.5. Classification when $\operatorname{dim} T=\operatorname{dim} M-2$. Then $M / T$ is two-dimensional. This is the only case in which we can give a classification, thanks to the following (for a proof see [128, Section 9.5]).

Lemma 8.3 (Thurston). Given a positive integer $g$ and an $m$-tuple $\left(o_{k}\right)_{k=1}^{m}$, $o_{i} \leqslant o_{i+1}$ of positive integers, there is a compact connected boundaryless orientable smooth orbisurface $\mathcal{O}$ with underlying topological space a compact connected surface of genus $g$ and with $m$ cone points of respective orders $o_{1}, \ldots, o_{m}$. Secondly, let $\mathcal{O}, \mathcal{O}^{\prime}$ be compact connected boundaryless orientable smooth orbisurfaces. Then $\mathcal{O}$ is diffeomorphic to $\mathcal{O}^{\prime}$ if and only if the genera of their underlying surfaces are the same, and their associated increasingly ordered $m$-tuples of orders of cone points are equal.

Remark 8.4. In [153, Theorem 13.3.6] Thurston gives a geometric classification of orbisurfaces which considers hyperbolic, elliptic, and parabolic structures.

The tuple $(g ; \vec{o})$ is the Fuchsian signature of $\mathcal{O}$. If $(\mathcal{O}, \omega)$ is a symplectic orbisurface, $\int_{\mathcal{O}} \omega$ is the symplectic area of $(\mathcal{O}, \omega)$. It follows from Lemma 8.3 and the orbifold Moser theorem [114, Theorem 3.3] (for the manifold case, see Theorem 2.18) that if $\operatorname{dim} T=\operatorname{dim} M-2$, the Fuchsian signature and symplectic area of $M / T$ determine it up to symplectomorphisms.

Let $(g ; \vec{o}) \in \mathbb{Z}^{1+m}$ be the Fuchsian signature of $M / T$. Let $\left\{\gamma_{k}\right\}_{k=1}^{m}$ be a basis of small loops around the singular orbifold points $p_{1}, \ldots, p_{m}$ of $M / T$. Let $\left\{\alpha_{1}, \beta_{1}, \ldots, \alpha_{g}, \beta_{g}\right\}$ be a symplectic basis of a free subgroup $F$ of

$$
\mathrm{H}_{1}^{\text {orb }}(M / T ; \mathbb{Z})=\left\langle\left\{\alpha_{i}, \beta_{i}\right\}_{i=1}^{g},\left\{\gamma_{k}\right\}_{k=1}^{m} \mid \sum_{k=1}^{m} \gamma_{k}=0, o_{k} \gamma_{k}=0,1 \leqslant k \leqslant m\right\rangle
$$

whose direct sum with the torsion subgroup of $\mathrm{H}_{1}^{\text {orb }}(M / T ; \mathbb{Z})$ is $\mathrm{H}_{1}^{\text {orb }}(M / T ; \mathbb{Z})$.

Let $\mu_{\mathrm{h}}: \mathrm{H}_{1}^{\text {orb }}(M / T ; \mathbb{Z}) \rightarrow T$ be the homomorphism induced on homology by $\mu$ in (8.1). Let $\operatorname{Sp}(2 g, \mathbb{Z})$ be the group of $2 g$-dimensional symplectic matrices with integer entries, and let $\mathcal{M} \mathrm{S}_{m}^{\vec{o}}$ be the group of $m$-dimensional matrices $D \in$ $\operatorname{GL}(m ; \mathbb{Z})$, such that $D(\vec{o})=\vec{o}$. The monodromy invariant is the equivalence class of $\left(\left(\mu_{\mathrm{h}}\left(\alpha_{i}\right), \mu_{\mathrm{h}}\left(\beta_{i}\right)\right)_{i=1}^{g},\left(\mu_{\mathrm{h}}\left(\gamma_{k}\right)\right)_{k=1}^{m}\right)$ by the relation: two tuples are related when they are taken to each other by a matrix of the form

$$
\left(\begin{array}{cc}
A & 0 \\
C & D
\end{array}\right) \in \mathrm{GL}(2 g+m ; \mathbb{Z})
$$

with $A \in \operatorname{Sp}(2 g, \mathbb{Z})$ and $D \in \mathcal{M} \mathrm{S}_{m}^{\vec{o}}$. Even though the monodromy invariant depends on choices, one can show that it is well defined; essentially $\operatorname{Sp}(2 g, \mathbb{Z})$ accounts for the freedom of choice of symplectic basis, and $\mathcal{M} \mathrm{S}_{m}^{\vec{o}}$ for the fact that the orders of the orbifold points may be permuted.

Theorem 8.5 ([128]). Compact connected symplectic manifolds $(M, \omega)$ with a maximal symplectic $T$-action such that $\operatorname{dim} T=\operatorname{dim} M-2$ are classified up to T-equivariant symplectomorphisms by the following symplectic invariants: the fundamental form $\omega^{\mathrm{t}}$, the Fuchsian signature $(g ; \vec{o})$ of $M / T$, the symplectic area $\lambda$ of $M / T$, and the monodromy invariant. Moreover, for any such list $\mathcal{L}$ of four invariants there exists a compact connected symplectic manifold $\left(M_{\mathcal{L}}, \omega_{\mathcal{L}}\right)$ with a maximal symplectic $T$-action of a $\left(\operatorname{dim} M_{\mathcal{L}}-2\right)$-torus $T$ with $\left(\operatorname{dim} M_{\mathcal{L}}-2\right)$-dimensional symplectic $T$-orbits whose list of invariants is $\mathcal{L}$. 
Theorem 8.5 is an extension of Theorem 6.4 to a class of non-Hamiltonian symplectic actions. The first part of Theorem 8.5 is uniqueness. The second part is existence, for which we have not provided details for simplicity; this would amount to saying which form $\omega^{\mathfrak{t}}$, etc., can appear. We shall say, however, that any antisymmetric bilinear form can appear, essentially all tuples $(g ; \vec{o})$ appear (with few exceptions, to account for the fact that $M / T$ be a good orbifold), and any $\lambda>0$ can appear. Similarly for the monodromy invariant. Readers may consult [128] for the precise list.

From this theorem one can derive topological consequences; for instance in 43. it is shown that the first Betti number of $M / T$ is equal to the first Betti number of $M$ minus the dimension of $T$.

8.2. Classification of coisotropic actions. This section gives symplectic invariants of coisotropic actions, as in Definition 7.6. Using these invariants, we construct a model of $(M, \omega)$ and the $T$-action, and we give a classification in terms of these invariants up to $T$-equivariant symplectomorphisms (which includes, as a particular case, the classification of symplectic-toric manifolds in Theorem 6.4). Let $(M, \omega)$ be a compact connected symplectic manifold with a coisotropic $T$-action of a torus $T$.

8.2.1. Hamiltonian action. A coisotropic $T$-action on $(M, \omega)$ is in general not Hamiltonian (see for instance Examples 5.9, 5.10, and 5.11). In this section we determine the maximal subtorus of $T$ which acts in a Hamiltonian manner on $(M, \omega)$, which may or may not be trivial. Let $x \in M$. By Lemma 7.13, $T_{x}$ is a subtorus of $T$. Let $m=\operatorname{dim} T_{x}$. Let $K$ be a complementary subtorus of $T_{x}$ in $T$. For $t \in T$, let $t_{x}$ and $t_{K}$ be the unique elements in $T_{x}$ and $K$, respectively, such that $t=t_{x} t_{K}$.

The inner product $\beta_{j}$ on $E_{j}$, in the proof of Lemma 7.13 , is unique if one requires that the symplectic inner product of any orthonormal basis with respect to $\omega^{W}$ is \pm 1 . This leads to the existence of a unique complex structure on $E_{j}$ such that, for any unit vector $e_{j}$ in $\left(E_{j}, \beta_{j}\right)$, we have that $e_{j}, \mathrm{i} e_{j}$ is an orthonormal basis in $\left(E_{j}, \beta_{j}\right)$ and $\omega^{W}\left(e_{j}, \mathrm{i} e_{j}\right)=1$. This leads to an identification of $E_{j}$ with $\mathbb{C}$, and hence of $W$ with $\mathbb{C}^{m}$, with symplectic form $\omega^{\mathbb{C}^{m}}=\frac{1}{2 \mathrm{i}} \sum_{j=1}^{m} \mathrm{~d} \overline{z^{j}} \wedge \mathrm{d} z^{j}$. The element $c \in \mathbb{T}^{m}=\left(S^{1}\right)^{m}$ acts on $\mathbb{C}^{m}$ componentwise by $(c \cdot z)^{j}=c^{j} z^{j}$. There is a unique Lie group isomorphism $\iota: T_{x} \rightarrow \mathbb{T}^{m}$ such that $h \in T_{x}$ acts on $W=\mathbb{C}^{m}$ by sending $z \in \mathbb{C}^{m}$ to $\iota(h) \cdot z$.

Recall that $\mathfrak{l}=\operatorname{ker}\left(\omega^{\mathfrak{t}}\right)$, where $\omega^{\mathfrak{t}}$ is the fundamental form (Proposition 7.1) and $X_{\mathfrak{l}}$ is the projection of $X \in \mathfrak{t}$ onto $\mathfrak{l}$. Let $T$ act on $K \times\left(\mathfrak{l} / \mathfrak{t}_{x}\right)^{*} \times \mathbb{C}^{m}$ by $t \cdot(k, \lambda, z)=\left(t_{K} k, \lambda, \iota\left(t_{x}\right) \cdot z\right)$. Endow this product with the symplectic form given at a point $(k, \lambda, z)$, and vectors $\left((X, \delta \lambda, \delta z),\left(X^{\prime}, \delta^{\prime} \lambda, \delta^{\prime} z\right)\right)$, by the formula:

$$
\omega^{\mathfrak{t}}\left(X, X^{\prime}\right)+\delta \lambda\left(X_{\mathfrak{l}}^{\prime}\right)-\delta^{\prime} \lambda\left(X_{\mathfrak{l}}\right)+\omega^{\mathbb{C}^{m}}\left(\delta z, \delta^{\prime} z\right) .
$$

Theorem 7.2 implies a symplectic normal form theorem for coisotropic actions:

Lemma 8.6 (Benoist). Let $x \in M$. Then there exists an open $\mathbb{T}^{m}$-invariant neighborhood $V$ of $(0,0)$ in $\left(\mathfrak{l} / \mathfrak{t}_{x}\right)^{*} \times \mathbb{C}^{m}$, an open $T$-invariant neighborhood $U_{x}$ of $x$ in $M$, and a T-equivariant symplectomorphism $\Phi: K \times V \rightarrow U_{x}$ such that $\Phi(1,0,0)=x$.

Using Benoist's result, one can detect the maximal subtorus that acts on $(M, \omega)$ in a Hamiltonian manner. 
Proposition 8.7. Let $(M, \omega)$ be a compact connected symplectic manifold endowed with a coisotropic action of a torus $T$. Then the product of all stabilizer subgroups $T_{x}, x \in M$, of the T-action, is a subtorus of $T$, denoted by $T_{\mathrm{h}}$. Moreover, the restriction of the $T$-action to $T_{\mathrm{h}}$ is a Hamiltonian action on $(M, \omega)$. Furthermore, the restriction of the $T$-action to any complementary subtorus $T_{\mathrm{f}}$ to $T_{\mathrm{h}}$ in $T$ is a free symplectic action on $(M, \omega)$.

Proof. By Lemma 8.6 the stabilizer subgroup of the $T$-action on $K \times\left(\mathfrak{l} / \mathfrak{t}_{x}\right)^{*} \times \mathbb{C}^{m}$ at $(k, \lambda, z)$ consists of the $t_{x} \in T_{x}$ for which $\iota\left(t_{x}\right)^{j}=1$ for all $j$ such that $z^{j} \neq 0$, and there are $2^{m}$ different stabilizer subgroups $T_{y}, y \in U_{x}$. Since $M$ is compact, there are only finitely many different stabilizer subgroups of $T$. The product of all the different stabilizer subgroups is a subtorus of $T$ because $T_{x}$ is a subtorus for every $x$ (Lemma 7.13), and the product of finitely many subtori is a compact and connected subgroup. See [40, Corollary 3.11] for the second claim. The torus $T_{\mathrm{f}}$ acts freely on $M$, because if $x \in M$, then $T_{x} \subset T_{\mathrm{h}}$, hence $T_{x} \cap T_{\mathrm{f}} \subset T_{\mathrm{h}} \cap T_{\mathrm{f}}=\{1\}$.

Koszul's tube theorem [100] ([39, Theorem 2.4.1]) also implies that there are finitely many stabilizer subgroups in the proof of Proposition 8.7. The torus $T_{\mathrm{h}}$ is the unique maximal stabilizer subgroup of $T$. Indeed, the $T_{\mathrm{h}}$-action has fixed points (for instance, by Theorem 4.3). By Proposition 8.7 there is $x_{0} \in M$ with $T_{\mathrm{h}} \subset T_{x_{0}}$, so $T_{\mathrm{h}}=T_{x_{0}}$ because $T_{x} \subset T_{\mathrm{h}}$ for every $x \in M$.

8.2.2. Orbit space. Because the symplectic $T$-action on $M$ may not be free in general (Example 5.11), $M / T$ is a singular space. Next we discuss more precisely the nature of its singularities. First, the regular part $M_{\mathrm{reg}} / T$ of $M / T$ is a smooth manifold of dimension $\operatorname{dim} M-\operatorname{dim} T$, and $\left.\pi\right|_{M_{\text {reg }}}: M_{\text {reg }} \rightarrow M_{\text {reg }} / T$ is a principal $T$-bundle. If $x \in M^{\mathrm{reg}}, \mathfrak{t}_{x}=\{0\}$, and $\mathfrak{l} \simeq\left(\mathrm{T}_{x}(T \cdot x)\right)^{\omega_{x}} \subset \mathrm{T}_{x}(T \cdot x) \simeq \mathfrak{t}$. Therefore $\operatorname{dim}\left(M_{\mathrm{reg}} / T\right)=\operatorname{dim} \mathfrak{l}$.

If $X \in \mathfrak{l}, \widehat{\omega}(X):=\mathrm{i}_{X_{M}} \omega$ is a closed basic 1-form on $M$ and $\widehat{\omega}_{x}: X \mapsto \widehat{\omega}(X)_{x}$ is an $\mathfrak{l}^{*}$-valued linear form on $\mathrm{T}_{x} M$, then $\widehat{\omega}: x \mapsto \widehat{\omega}_{x}$ is a basic closed $\mathfrak{l}^{*}$-valued 1-form on $M$. In the normal form Lemma 8.6 with $x \in M_{\text {reg }}, \mathfrak{t}_{x}=\{0\}, m=0, \widehat{\omega}$ is $(\delta t, \delta \lambda) \mapsto \delta \lambda: \mathfrak{t} \times \mathfrak{l}^{*} \rightarrow \mathfrak{l}^{*}$ at each point. Hence, if $p \in M_{\mathrm{reg}} / T$, the induced form $\widehat{\omega}_{p}: \mathrm{T}_{p}\left(M_{\mathrm{reg}} / T\right) \rightarrow \mathfrak{l}^{*}$ is a linear isomorphism. In this way, $\xi \in \mathfrak{l}^{*}$ may be viewed as a constant vector field. It follows from the closedness of $\widehat{\omega}$ that $M_{\text {reg }} / T$ may be provided with an atlas in $\mathfrak{l}^{*}$ with tangent map $\widehat{\omega}$, and two local charts differ by a translation in $\mathfrak{l}^{*}$.

Moreover, using Lemma 8.6, one can show that the entire $M / T$ has the structure of an $\mathfrak{l}^{*}$-parallel space, which intuitively is a space modeled on $\mathfrak{l}^{*}$. The detailed structure of what is called a $V$-parallel space (in our case $V=\mathfrak{l}^{*}$ ) is beyond the scope of this paper. We refer to [40, Section 11] where it is proved that any such space, for any vector space $V$, is homeomorphic, by means of a homeomorphism that preserves the $\mathfrak{l}^{*}$-structure, to the product of a closed convex set and a torus.

Let $\mathfrak{t}_{\mathrm{h}}$ be the Lie algebra of $T_{\mathrm{h}}$. By Proposition 8.7 the $T_{\mathrm{h}}$-action on $(M, \omega)$ is Hamiltonian, so it has a momentum map

$$
\mu: M \rightarrow\left(\mathfrak{t}_{\mathrm{h}}\right)^{*},
$$

and its image $\mu(M)$ is, by the Atiyah-Guillemin-Sternberg convexity theorem (Theorem 4.3), a convex polytope $\Delta \subset \mathfrak{t}_{\mathrm{h}}{ }^{*}$. Next we explain how, in the case of the $\mathfrak{l}^{*}$-parallel space $M / T$, the aforementioned closed convex set is precisely $\Delta$ in (8.3). 
If $p \in M / T$ is not regular, there is an integral basis $\left\{X_{j}\right\}_{j \in J}$, of the integral lattice of $T_{x}$ in $\mathfrak{t}_{x}$ such that near $p$, the chart of $M / T$ is identified with a corner $\bigcap_{j \in J}\left\{\xi \in \mathfrak{l}^{*} \mid \xi\left(X_{j}\right) \geqslant 0\right\}$. If $\zeta$ is an element of the additive group

$$
N:=\left(\mathfrak{l} / \mathfrak{t}_{\mathrm{h}}\right)^{*},
$$

a linear form on $\mathfrak{l}$ which is zero on $\mathfrak{t}_{\mathrm{h}}$; i.e., on every $\mathfrak{t}_{x}$, the straight line solution curves of the constant vector field $\zeta$ on $M / T$ do not meet the nonregular points of $M / T$ so that there is a global action, denoted by $(\zeta, p) \mapsto p+\zeta$, of $N$ on $M / T$, by traveling for time 1 along the solution curve. The period group $P \subset N$ of this action is a cocompact discrete subgroup of $N$, and $N / P$ is a torus of dimension $\operatorname{dim} N$. A detailed analysis allows one to verify that $\Phi_{p}(\eta, \zeta):=p+(\eta+\zeta)$ is an isomorphism between the $\mathfrak{l}^{*}$-parallel spaces $\Delta \times(N / P)$ and $M / T$. In particular:

Proposition 8.8. Let $(M, \omega)$ be a compact connected symplectic manifold endowed with a coisotropic action of a torus $T$. Let $\mathfrak{l}$ be the kernel of the fundamental form $\omega^{\mathrm{t}}$, and let $T_{\mathrm{h}}$ be the largest subtorus of $T$ which acts on $(M, \omega)$ in a Hamiltonian fashion. Then the orbit space $M / T$ is homeomorphic to the product of a Delzant polytope $\Delta$ of dimension $\operatorname{dim} T_{\mathrm{h}}$ and a torus $S$ of dimension $\operatorname{dim} \mathfrak{l}-\operatorname{dim} T_{\mathrm{h}}$.

The composite $M \rightarrow \mathfrak{t}_{\mathrm{h}}{ }^{*}$ of $\pi: M \rightarrow M / T$, followed by $\Phi_{p}^{-1}: M / T \rightarrow \Delta \times(N / P)$, the projection $\Delta \times(N / P) \rightarrow \Delta$, and the inclusion $\Delta \hookrightarrow\left(\mathfrak{t}_{\mathrm{h}}\right)^{*}$, is a momentum mapping for the Hamiltonian $T_{\mathrm{h}}$-action on $M$ in Proposition 8.7 and is equal to $\mu$ in (8.3) up to an additive constant.

The orbit map $\pi: M \rightarrow M / T$ followed by $\Phi_{p}^{-1}: M / T \rightarrow \Delta \times(N / P)$ and the projection $\Delta \times(N / P) \rightarrow N / P$ is a torus-valued generalization of the $S^{1}$-momentum map of McDuff [115.

8.2.3. A singular connection. A smooth vector field $L_{\zeta}$ on $M_{\text {reg }}$ is a lift of $\zeta$ if $\mathrm{d}_{x} \pi\left(L_{\zeta}(x)\right)=\zeta$ for all $x \in M_{\text {reg. }}$. The word "lift" is in the sense that $\zeta \in \mathfrak{l}^{*}$ is a constant vector field on $(M / T)_{\text {reg }}$ of which $L_{\zeta}$ is a lift. Assignments of lifts $\zeta \in \mathfrak{l}^{*} \mapsto L_{\zeta}$, which depend linearly on $\zeta$, and connections for $M_{\text {reg }} \rightarrow M_{\text {reg }} / T$ are equivalent objects. If $\mathfrak{t}_{\mathrm{f}}$ is the Lie algebra of $T_{\mathrm{f}}$ in Proposition 8.7, then $\mathfrak{t}=\mathfrak{t}_{\mathrm{h}} \oplus \mathfrak{t}_{\mathrm{f}}$. A step for the construction of the model of $(M, \omega)$ with $T$-action is the existence of the following connection, where $C$ is such that $C \oplus N=\mathfrak{l}^{*}$, i.e., it is a linear complement of $N$ in $\mathfrak{l}^{*}$.

Lemma 8.9. There exists an antisymmetric bilinear map $c: N \times N \rightarrow \mathfrak{l}$, satisfying $\zeta\left(c\left(\zeta^{\prime}, \zeta^{\prime \prime}\right)\right)+\zeta^{\prime}\left(c\left(\zeta^{\prime \prime}, \zeta\right)\right)+\zeta^{\prime \prime}\left(c\left(\zeta, \zeta^{\prime}\right)\right)=0$ if $\zeta, \zeta^{\prime}, \zeta^{\prime \prime} \in N$, and a T-invariant connection

$$
\zeta \in \mathfrak{l}^{*} \mapsto L_{\zeta},
$$

of smooth vector fields $L_{\zeta}$ on $M_{\mathrm{reg}}$, whose Lie brackets satisfy

$$
\left[L_{\zeta}, L_{\eta}\right]=c(\zeta, \eta)_{M}, \zeta, \eta \in N
$$

$\left[L_{\eta}, L_{\eta^{\prime}}\right]=0$ if $\eta, \eta^{\prime} \in C$, and $\left[L_{\eta}, L_{\zeta}\right]=0$ if $\eta \in C, \zeta \in N$. Moreover, if $c_{\mathrm{h}}(\zeta, \eta)$ is the $\mathfrak{t}_{\mathrm{h}}$-component of $c(\zeta, \eta)$ in $\mathfrak{l}=\mathfrak{t}_{\mathrm{h}} \oplus\left(\mathfrak{l} \cap \mathfrak{t}_{\mathrm{f}}\right)$, then the symplectic form $\omega$ satisfies

$$
\omega_{x}\left(L_{\zeta}(x), L_{\eta}(x)\right)=-\underbrace{\mu(x)}_{\in\left(\mathfrak{t}_{\mathrm{h}}\right)^{*}}(\underbrace{c_{\mathrm{h}}(\zeta, \eta)}_{\in \mathfrak{t}_{\mathrm{h}}}), \zeta, \eta \in N, x \in M
$$

as well as $\omega\left(L_{\eta}, L_{\eta^{\prime}}\right)=0$ if $\eta, \eta^{\prime} \in C$ and $\omega\left(L_{\eta}, L_{\zeta}\right)=0$ if $\eta \in C, \zeta \in N$. 


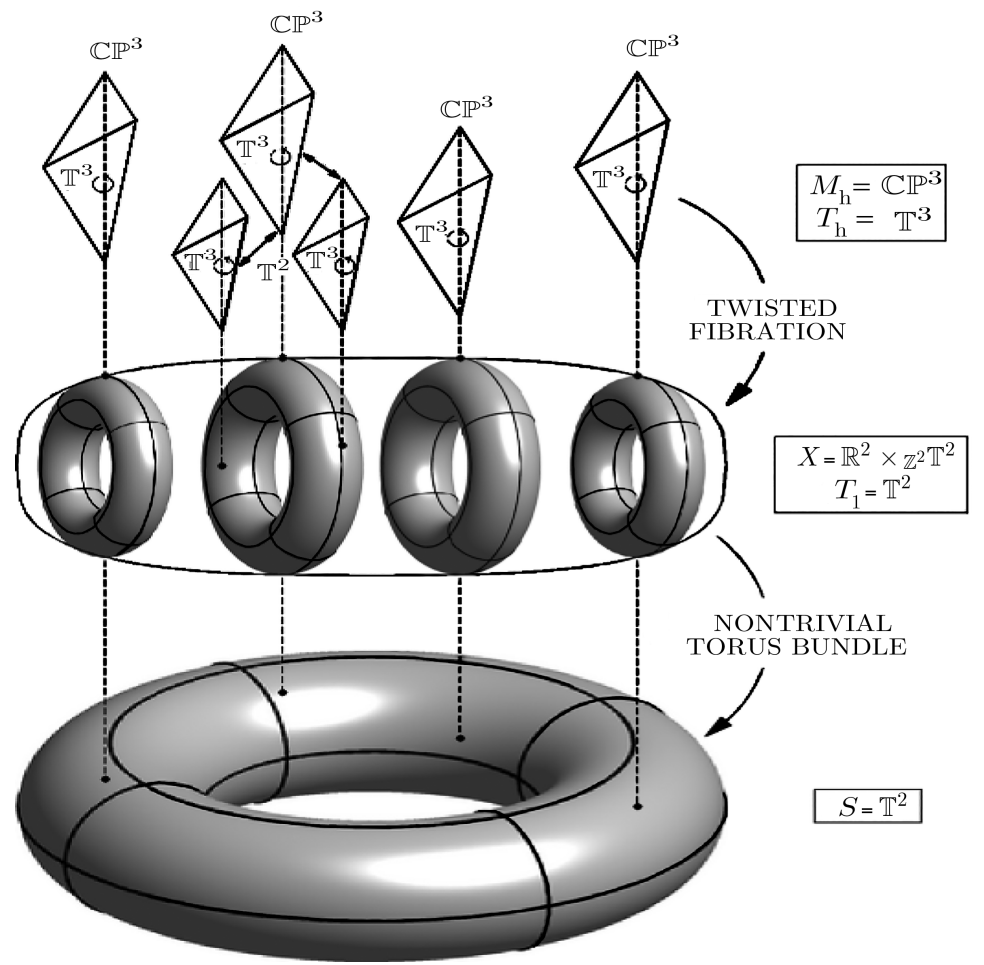

Figure 4. Let $\mathbb{T}^{k}:=\left(\mathbb{R}^{k} / \mathbb{Z}^{k}\right)$. The figure shows a typical coisotropic $T$-action (Definition [7.6) of a five-dimensional torus $T=\mathbb{T}^{5}$ on a compact connected symplectic ten-dimensional manifold $(M, \omega)$. The principal $T$-orbits are Lagrangian fivedimensional submanifolds of $(M, \omega)$. The figure depicts the fibration in Theorem 8.11 the fiber is the six-dimensional symplectictoric manifold $\left(M_{\mathrm{h}}, \omega_{\mathrm{h}}, T_{\mathrm{h}}\right)=\mathbb{C P}^{3}$ with the Fubini-Study form (Example 5.2) and the standard Hamiltonian action of the standard 3 -torus $T_{\mathrm{h}}=\mathbb{T}^{3}$; the base $G \times_{H} M_{\mathrm{h}}$ of the fibration is the 2-torus bundle $X=G / H=\mathbb{R}^{2} \times_{\mathbb{Z}^{2}} \mathbb{T}^{2}$ over $\mathbb{T}^{2}$. According to Delzant (Theorem 6.4), $\left(M_{\mathrm{h}}, \omega_{\mathrm{h}}, T_{\mathrm{h}}\right)$ is determined up to equivariant symplectomorphisms by its image under the momentum map, which by formula (5.1) is the standard three-dimensional simplex, and hence why we have represented the six-dimensional fibers in the figure by three-dimensional simplices. The 2-torus $T_{\mathrm{f}}=\mathbb{T}^{2}$, which is complementary to the Hamiltonian torus $T_{\mathrm{h}}=\mathbb{T}^{3}$, acts freely on $(M, \omega)$ by permuting the fibers. The picture illustrates the fact that a coisotropic $T$-action exhibits both Hamiltonian features (encoded by the fibers $M_{\mathrm{h}}$ ) and non-Hamiltonian features (encoded by the base $X=G / H$ ) of the fibration). When the base is trivial the $T$-action on $M$ is Hamiltonian; when the fibers are trivial the $T$-action on $M$ is free and non-Hamiltonian (see Remark 8.12). 
The connection (8.5) blows up at $M \backslash M_{\text {reg. }}$. If $\zeta \in N, L_{\zeta}$ has a smooth extension to $M$.

The map $c$ in Lemma 8.9 has a geometric interpretation, which we discuss next. There is an isomorphism $M / T_{\text {reg }} \simeq \operatorname{Interior}(\Delta) \times(N / P)$, induced by $\Phi_{p}^{-1}$. Any connection for the principal $T$-bundle $M_{\text {reg }} \rightarrow M_{\text {reg }} / T$ has a curvature form, a smooth t-valued 2-form on $M_{\text {reg }} / T$. Its cohomology class is an element of $\mathrm{H}^{2}\left(M_{\mathrm{reg}} / T ; \mathfrak{t}\right)$, independent of the choice of the connection. The $N$-action on $M / T$ leaves $M_{\text {reg }}$ invariant, with orbits isomorphic to $N / P$. The pullback to the $N$-orbits defines an isomorphism from $\mathrm{H}^{2}\left(M_{\mathrm{reg}} / T ; \mathfrak{t}\right)$ to $\mathrm{H}^{2}(N / P ; \mathfrak{t})$, which is identified with $\left(\Lambda^{2} N^{*}\right) \otimes \mathfrak{t}$ (this goes back to É. Cartan). It follows from the construction of (8.5) that $c: N \times N \rightarrow \mathfrak{l}$, viewed as $c \in\left(\Lambda^{2} N^{*}\right) \otimes \mathfrak{l} \subset\left(\Lambda^{2} N^{*}\right) \otimes \mathfrak{t}$, is the negative of the pullback to an $N$-orbit of the cohomology class of the curvature form. Hence $c: N \times N \rightarrow \mathfrak{l}$ in (8.6) is independent of $T_{\mathrm{f}}$. The Chern class $\mathcal{C}$ of $\pi: M_{\mathrm{reg}} \rightarrow M_{\mathrm{reg}} / T$ is an element of $\mathrm{H}^{2}\left(M_{\mathrm{reg}} / T ; T_{\mathbb{Z}}\right)$. The image of $\mathcal{C}$ under the coefficient homomorphism $\mathrm{H}^{2}\left(M_{\mathrm{reg}} / T ; T_{\mathbb{Z}}\right) \rightarrow \mathrm{H}^{2}\left(M_{\mathrm{reg}} / T ; \mathfrak{t}\right)$ is the negative of the cohomology class of the curvature form of any connection in the principal $T$-bundle, and hence $c: N \times N \rightarrow \mathfrak{l}$ represents $\mathcal{C}$.

8.2.4. Foliation by symplectic-toric manifolds. See Section 6.1 to review symplectictoric manifolds. Let $L_{\eta}$ be as in Lemma 8.9

Proposition 8.10. There exists a unique smooth $T$-invariant distribution $\mathcal{D}:=$ $\left\{D_{x}\right\}_{x \in M}$ on $M$ such that for each $x \in M_{\text {reg }}$,

$$
D_{x}=\operatorname{span}\left\{L_{\eta}(x), Y_{M}(x) \mid Y \in \mathfrak{t}_{\mathrm{h}}, \eta \in C\right\} .
$$

The integral manifolds of $\mathcal{D}$ are smooth $\left(2 \operatorname{dim} T_{\mathrm{h}}\right)$-dimensional manifolds. We pick an integral manifold and call it $M_{\mathrm{h}}$. Then $\omega$ restricts to a symplectic form, say $\omega_{\mathrm{h}}$, on $M_{\mathrm{h}}$, and $T_{\mathrm{h}}$ acts in a Hamiltonian manner on $M_{\mathrm{h}}$, that is, $\left(M_{\mathrm{h}}, \omega_{\mathrm{h}}, T_{\mathrm{h}}\right)$ is a symplectic-toric manifold. All integral manifolds of $\mathcal{D}$, with the restriction of $\omega$, are $T_{\mathrm{h}}$-equivariantly symplectomorphic to $\left(M_{\mathrm{h}}, \omega_{\mathrm{h}}, T_{\mathrm{h}}\right)$.

The integral manifolds of $\mathcal{D}$ are depicted in Figure 4 as polytopes (the fibers of the fibration therein) because of the correspondence established by Delzant (Theorem 6.4) between symplectic-toric manifolds and Delzant polytopes.

8.2.5. Group extensions and the holonomy invariant. Denote the flow after time $t \in \mathbb{R}$ of a vector field $\mathcal{X}$ on $M$ by $\mathrm{e}^{t \mathcal{X}}$. The extension of $N$ by $T$ is the Lie group

$$
G:=T \times N
$$

with operation

$$
(t, \zeta)\left(t^{\prime}, \eta\right)=\left(t t^{\prime} \mathrm{e}^{-c(\zeta, \eta) / 2}, \zeta+\eta\right)
$$

The Lie group $G$ acts smoothly on $M$ by $(t, \zeta) \mapsto t_{M} \circ \mathrm{e}^{L_{\zeta}}$, where we are using the identification $G \simeq\left(\mathfrak{t} / T_{\mathbb{Z}}\right) \times N$. The projection $\pi: M \rightarrow M / T$ intertwines the action of $G$ on $M$ with the action of $N$ on $M / T$, and there is an exact sequence $1 \rightarrow T \rightarrow G \rightarrow N \rightarrow 1$, where $G \rightarrow N$ corresponds to passing from the action of $G$ on $M$ to the action of $N$ on $M / T$, on which the action of $T$ is trivial. One can show that the Lie algebra of $G$ with (8.7) is the two-step nilpotent Lie algebra $\mathfrak{g}=\mathfrak{t} \times N$ with $\left[(X, \zeta),\left(X^{\prime}, \eta\right)\right]=-(c(\zeta, \eta), 0)$. The product $\mathfrak{t} \times N$ endowed with $(X, \zeta)\left(X^{\prime}, \eta\right)=\left(X+X^{\prime}-c(\zeta, \eta) / 2, \zeta+\eta\right)$ is a two-step nilpotent Lie group with Lie algebra $\mathfrak{g}$, and the identity as the exponential map. 
For $\zeta \in P$ and $p \in M / T$ consider the loop $\gamma_{\zeta}(t):=p+t \zeta$. If $p=\pi(x)$, $\delta(t)=\mathrm{e}^{t L_{\zeta}}(x), t \in[0,1]$, is the horizontal lift of $\gamma_{\zeta}$ which starts at $x$ because $\delta(0)=x$ and $\delta^{\prime}(t)=L_{\zeta}(\delta(t))$ is a tangent vector mapped by $\mathrm{d}_{\delta(t)} \pi$ to $\zeta$. Hence $\pi(\delta(t))=\gamma_{\zeta}(t)$ if $t \in[0,1]$. The element of $T$ which maps $\delta(0)=x$ to $\delta(1)$ is the holonomy $\tau_{\zeta}(x)$ of the loop $\gamma_{\zeta}$ at $x$ with respect to the connection (8.5). Because $\delta(1)=\mathrm{e}^{L_{\zeta}}(x)$, we have $\tau_{\zeta}(x) \cdot x=\mathrm{e}^{L_{\zeta}}(x)$. The element $\tau_{\zeta}(x)$ depends on $x \in M$, the period $\zeta \in P$, and the choice of connection (8.5). Let

$$
H:=\left\{(t, \zeta) \in G \mid \zeta \in P, t \tau_{\zeta} \in T_{\mathrm{h}}\right\} .
$$

The elements $\tau_{\zeta} \in T, \zeta \in P$, encode the holonomy of (8.5). The holonomy is an element of the set $\operatorname{Hom}_{c}(P, T)$ of maps $\tau: P \rightarrow T$, denoted by $\zeta \mapsto \tau_{\zeta}$, such that $\tau_{\zeta} \tau_{\eta}=\tau_{\zeta+\eta} \mathrm{e}^{c(\eta, \zeta) / 2}$. There is a Lie subgroup $B \leqslant \operatorname{Hom}_{c}(P, T)$ which eliminates the dependence on the choice of connection and basepoint, so the true holonomy invariant of $(M, \omega)$ is an element of $\operatorname{Hom}_{c}(P, T) / B$. The precise definition of $B$ is technical, and it appeared in 40.

The map

$$
((t, \zeta), x) \mapsto\left(t \tau_{\zeta}\right) \cdot x: H \times M_{\mathrm{h}} \rightarrow M_{\mathrm{h}}
$$

is a smooth action of $H$ on the symplectic toric manifold $M_{\mathrm{h}}$.

8.2.6. The relation with nilmanifolds. The quotient $G / H$ is taken with respect to the nonstandard group structure in expression (8.7). On $G / H$ we still have the free action of the torus $T / T_{\mathrm{h}}$, which exhibits $G / H$ as a principal $T / T_{\mathrm{h}}$-bundle over the torus $(G / H) / T \simeq N / P$. Palais and Stewart 124. showed that every principal torus bundle over a torus is diffeomorphic to a nilmanifold for a two-step nilpotent Lie group. When the nilpotent Lie group is not Abelian, the manifold $M$ does not admit a Kähler structure; see Benson and Gordon [17]. In fact, the $G$-space $G / H$ is isomorphic to the quotient of the simply connected two-step nilpotent Lie group $\left(\mathfrak{t} / \mathfrak{t}_{\mathrm{h}}\right) \times N$ by the discrete subgroup of elements $(Z, \zeta)$ such that $\mathrm{e}^{Z} \tau_{\zeta} \in T_{\mathrm{h}}$. Indeed, the identity component $H^{o}=T_{\mathrm{h}} \times\{0\}$ of $H$ is a closed normal Lie subgroup of $G$ and $H$. The map $\left(G / H^{o}\right) /\left(H / H^{o}\right) \rightarrow G / H,\left(g H^{o}\right)\left(H / H^{o}\right) \mapsto g H$, is a $G$ equivariant diffeomorphism. The structure of $G / H^{o}=\left(T / T_{\mathrm{h}}\right) \times N$ is $(t, \zeta)\left(t^{\prime}, \eta\right)=$ $\left(t t^{\prime} \mathrm{e}^{-c_{\mathfrak{l} / \mathfrak{t}_{\mathrm{h}}}(\zeta, \eta) / 2}, \zeta+\eta\right), t, t^{\prime} \in T / T_{\mathrm{h}}, \zeta, \eta \in N$, where $c_{\mathfrak{l} / \mathfrak{t}_{\mathrm{h}}}: N \times N \rightarrow \mathfrak{l} / \mathfrak{t}_{\mathrm{h}}$ is the composite of $c: N \times N \rightarrow \mathfrak{l}$ and the projection $\mathfrak{l} \rightarrow \mathfrak{l} / \mathfrak{t}_{\mathrm{h}}$. Hence $G / H^{o}$ is a twostep nilpotent Lie group with universal covering $\left(\mathfrak{t} / \mathfrak{t}_{\mathrm{h}}\right) \times N$ and covering group $\left(T / T_{\mathrm{h}}\right)_{\mathbb{Z}} \simeq T_{\mathbb{Z}} /\left(T_{\mathrm{h}}\right)_{\mathbb{Z}}$. Also, $P \rightarrow H / H^{o}$ given by $\iota: \zeta \mapsto\left(\tau_{\zeta}^{-1}, \zeta\right) H^{o}$ is an isomorphism.

8.2.7. Model. Let $h \in H$ act on $G \times M_{\mathrm{h}}$ by $h(g, x)=\left(g h^{-1}, h \cdot x\right)$, and consider the quotient $G \times_{H} M_{\mathrm{h}}$, where we recall that $h \cdot x$ is given in (8.8). The $T$-action by translations on the left factor of $G$ passes to an action on $G \times{ }_{H} M_{\mathrm{h}}$. Each of the fibers of $G \times_{H} M_{\mathrm{h}}$ is identified with the symplectic-toric manifold $\left(M_{\mathrm{h}}, \omega_{\mathrm{h}}, T_{\mathrm{h}}\right)$. Any complementary subtorus $T_{\mathrm{f}}$ permutes the fibers of $G \times_{H} M_{\mathrm{h}} \rightarrow G / H$.

Next we construct a symplectic form on $G \times_{H} M_{\mathrm{h}}$. This construction uses Lemma 8.6 but for simplicity we skip the details as the general formula may be given directly. Let $\delta a=((\delta t, \delta \zeta), \delta x)$ and $\delta^{\prime} a=\left(\left(\delta^{\prime} t, \delta^{\prime} \zeta\right), \delta^{\prime} x\right)$ be tangent vectors to $G \times M_{\mathrm{h}}$ at the point $a=((t, \zeta), x)$, where we identify each tangent space of $T$ with $\mathfrak{t}$. Write $X=\delta t+c(\delta \zeta, \zeta) / 2$ and $X^{\prime}=\delta^{\prime} t+c\left(\delta^{\prime} \zeta, \zeta\right) / 2$. Let $X_{\mathrm{h}}$ be the 
$\mathfrak{t}_{\mathrm{h}}$-component of $X \in \mathfrak{t}_{\text {in }} \mathfrak{t}_{\mathrm{h}} \oplus \mathfrak{t}_{\mathrm{f}}$, and similarly for $X_{\mathfrak{l}}$, and define

$$
\begin{aligned}
\Omega_{a}\left(\delta a, \delta^{\prime} a\right)= & \omega^{\mathfrak{t}}\left(\delta t, \delta^{\prime} t\right)+\delta \zeta\left(X_{\mathfrak{l}}^{\prime}\right)-\delta^{\prime} \zeta\left(X_{\mathfrak{l}}\right)-\mu(x)\left(c_{\mathrm{h}}\left(\delta \zeta, \delta^{\prime} \zeta\right)\right) \\
& +\left(\omega_{\mathrm{h}}\right)_{x}\left(\delta x,\left(X^{\prime}{ }_{\mathrm{h}}\right)_{M_{\mathrm{h}}}(x)\right)-\left(\omega_{\mathrm{h}}\right)_{x}\left(\delta^{\prime} x,\left(X_{\mathrm{h}}\right)_{M_{\mathrm{h}}}(x)\right)+\left(\omega_{\mathrm{h}}\right)_{x}\left(\delta x, \delta^{\prime} x\right) .
\end{aligned}
$$

If $\pi_{M}$ is the projection $G \times M_{\mathrm{h}} \rightarrow G \times{ }_{H} M_{\mathrm{h}}$, the $T$-invariant symplectic form on $G \times{ }_{H} M_{\mathrm{h}}$ is the unique 2-form $\tau$ on $G \times{ }_{H} M_{\mathrm{h}}$ such that $\Omega=\pi_{M}{ }^{*} \tau$.

A typical example of a ten-dimensional manifold in the following statement is given in Figure 4.

Theorem 8.11 (40]). A compact connected symplectic manifold $(M, \omega)$ with a coisotropic T-action is T-equivariantly symplectomorphic to the total space $G \times{ }_{H} M_{\mathrm{h}}$ of the fibration

$$
\left(M_{\mathrm{h}}, \omega_{\mathrm{h}}, T_{\mathrm{h}}\right) \hookrightarrow\left(G \times{ }_{H} M_{\mathrm{h}}, \tau, T\right) \rightarrow G / H
$$

with base $G / H$ being a torus bundle over a torus, and symplectic-toric manifolds $\left(M_{\mathrm{h}}, \omega_{\mathrm{h}}, T_{\mathrm{h}}\right)$ as fibers. The $T$-action on $G \times_{H} M_{\mathrm{h}}$ is the symplectic action by translations on the $T$-factor of $G$.

Using Lemmas 8.6 and 8.9 and Proposition 8.10, one can verify that $F: G \times{ }_{H}$ $M_{\mathrm{h}} \mapsto M, F((t, \xi), x)=t \cdot \mathrm{e}^{L_{\xi}}(x)$, is a $T$-equivariant diffeomorphism and $F^{*} \omega=\tau$. However, careful checking is fairly technical and is not necessarily illuminating on a first reading; we refer to [40] for a proof.

Remark 8.12. A extreme case of Theorem 8.11 occurs if the symplectic $T$-action is free. Then $T_{\mathrm{h}}$ is trivial and $M$ is the torus bundle $G / H$ over a torus (this is the base $X=G / H$ of the fibration in Figure 4). The Kodaira variety (Example 5.10) is one of these spaces. Since $M$ is a principal torus bundle over a torus, it is a nilmanifold for a two-step nilpotent Lie group by 124] (if the nilpotent Lie group is not Abelian, $M$ does not admit Kähler structures by [17]). If $\operatorname{dim} M=4$, this corresponds to the third case in Kodaira's description [96, Theorem 19] of the compact complex analytic surfaces which have a holomorphic $(2,0)$-form that is nowhere vanishing. As mentioned earlier, these were rediscovered by Thurston 152 as the first examples of compact symplectic manifolds without Kähler structure.

The other extreme case occurs if the symplectic $T$-action is Hamiltonian. Then $T_{\mathrm{h}}=T$, and $M=M_{\mathrm{h}}$ is a symplectic-toric manifold (Definition 6.2) and hence has the structure of a toric variety (see [33, 41,70, for the relations between symplectictoric manifolds and toric varieties).

8.2.8. Classification. The following is the classification of coisotropic actions.

Theorem 8.13 ([40]). Compact connected symplectic manifolds $(M, \omega)$ with a coisotropic T-action are classified up to T-equivariant symplectomorphisms by the symplectic invariants: the fundamental form $\omega^{\mathrm{t}}$, the Hamiltonian torus $T_{\mathrm{h}}$ and its associated polytope $\Delta$, the period lattice $P$ of $N=\left(\mathfrak{l} / \mathfrak{t}_{\mathrm{h}}\right)^{*}$, the Chern class $c: N \times N \rightarrow \mathfrak{l}$ of $M_{\mathrm{reg}} \rightarrow M_{\mathrm{reg}} / T$, and the holonomy invariant $[\tau: P \rightarrow T]_{B} \in$ $\operatorname{Hom}_{c}(P, T) / B$. Moreover, for any such list $\mathcal{L}$ of five invariants there exists a compact connected symplectic manifold $\left(M_{\mathcal{L}}, \omega_{\mathcal{L}}\right)$ with a coisotropic $T$-action with list of invariants $\mathcal{L}$.

The first part of Theorem 8.13 is a uniqueness result; the second part, an existence result (we have not provided details to preserve simplicity). Nonetheless we shall say that any antisymmetric bilinear form, any subtorus $S \subset T$, and any Delzant polytope may appear; details are in [40]. 
Example 8.14. For the Kodaira variety $M=\mathbb{R}^{2} \times_{\mathbb{Z}^{2}}(\mathbb{R} / \mathbb{Z})^{2}$ in Example $[5.10$, $T=(\mathbb{R} / \mathbb{Z})^{2}, \mathfrak{t} \simeq \mathbb{R}^{2}$ and its invariants are as follows: $\omega^{\mathfrak{t}}=0$; Hamiltonian torus $T_{\mathrm{h}}=\{[0,0]\}$; Delzant polytope $\Delta=\{(0,0)\}$; period lattice $P=\mathbb{Z}^{2}$; Chern class $c: \mathbb{R}^{2} \times \mathbb{R}^{2} \rightarrow \mathbb{R}^{2}, c\left(\mathrm{e}_{1}, \mathrm{e}_{2}\right)=\mathrm{e}_{1}$. The holonomy invariant is the class of $\tau$ given by $\tau_{\mathrm{e}_{1}}=\tau_{\mathrm{e}_{2}}=[0,0]$. In this case $G=(\mathbb{R} / \mathbb{Z})^{2} \times \mathbb{R}^{2}, M_{\mathrm{h}}$ is a point, and $H=\{[0,0]\} \times$ $\mathbb{Z}^{2}$. The model of $(M, \omega)$ in Theorem 8.11 is $G \times_{H} M_{\mathrm{h}} \simeq G / H \simeq \mathbb{R}^{2} \times_{\mathbb{Z}^{2}}(\mathbb{R} / \mathbb{Z})^{2}$.

The moduli space of coisotropic actions includes Hamiltonian actions of maximal dimension (see [129] for the description of this space in dimension 4), classified in Theorem 6.4

8.3. Classification of symplectic 2-torus actions on 4-manifolds. Consider on $(\mathbb{R} / \mathbb{Z})^{2} \times S^{2}$ a product symplectic form, and the action of the 2-torus where one circle acts on the first circle of $(\mathbb{R} / \mathbb{Z})^{2}$ by translation, while the other circle acts on $S^{2}$ by rotation about the vertical axis (this is Example [5.6, also depicted in Figure 2).

If $T$ is a two-dimensional torus, consider the product $T \times \mathfrak{t}^{*}$ with the standard cotangent bundle form and the standard $T$-action on left factor of $T \times \mathfrak{t}^{*}$.

The following is a simplified version of the main result of [128, Theorem 8.2.1].

Theorem 8.15 (128). Let $(M, \omega)$ be a compact connected symplectic 4-manifold equipped with an effective symplectic action of a 2-torus $T$. If the symplectic $T$ action is Hamiltonian, then:

(1) $(M, \omega)$ is a symplectic-toric 4-manifold, so classified up to T-equivariant symplectomorphisms by the image $\Delta$ of the momentum map $\mu: M \rightarrow \mathfrak{t}^{*}$ of the T-action (Theorem 6.4).

If the symplectic T-action is not Hamiltonian, then one and only one of the following cases occurs:

(2) $(M, \omega)$ is equivariantly symplectomorphic to $(\mathbb{R} / \mathbb{Z})^{2} \times S^{2}$.

(3) $(M, \omega)$ is equivariantly symplectomorphic to $\left(T \times \mathfrak{t}^{*}\right) / Q$ with the induced form and $T$-action, where $Q \leqslant T \times \mathfrak{t}^{*}$ is a discrete cocompact subgroup for the group structure (8.7) on $T \times \mathfrak{t}^{*}$.

(4) $(M, \omega)$ is equivariantly symplectomorphic to a symplectic orbifold bundle $\widetilde{\Sigma} \times_{\pi_{1}^{\text {orb }}\left(\Sigma, p_{0}\right)} T$ over a good orbisurface $\Sigma$, with symplectic form and $T$ action induced by the product ones. Here, in order to form the quotient $\widetilde{\Sigma} \times \pi_{1}^{\text {orb }(\Sigma)}$ T, the orbifold fundamental group $\pi_{1}^{\text {orb }}(\Sigma)$ acts on $\widetilde{\Sigma} \times T$ diagonally, and on $T$ by means of a homomorphism $\mu: \pi_{1}^{\text {orb }}(\Sigma) \rightarrow T$.

Idea of proof. By linear algebra either the T-orbits are symplectic 2-tori, so $\omega^{\mathrm{t}}$ is nondegenerate and $\mathfrak{l}$ is trivial (this is case (4)), or alternatively the two-dimensional $T$-orbits are Lagrangian 2-tori and $\mathfrak{l}=\mathfrak{t}$ (these are cases (1), (2), and (3)). Cases (2) and (3) are derived from Theorem 8.13. Case (4) is derived from Theorem 8.5. Case (1) is classified by $\Delta$ in view of Theorem 6.4 .

Case (3) appears in Kodaira [96, Theorem 19]; see Remark 8.12, Theorem 8.15] implies that the only compact connected symplectic 4-manifold with a nonlocally free and non-Hamiltonian effective symplectic action of a 2-torus is, up to equivariant symplectomorphisms, $(\mathbb{R} / \mathbb{Z})^{2} \times S^{2}$. 
Example 8.16. The invariants of $M=S^{2} \times_{\mathbb{Z} / 2 \mathbb{Z}}(\mathbb{R} / \mathbb{Z})^{2}$ in Example 5.11 are as follows: fundamental form $\left(\begin{array}{cc}0 & 1 \\ -1 & 0\end{array}\right)$; Fuchsian signature $(0 ; 2,2)$ of $M /(\mathbb{R} / \mathbb{Z})^{2}$; symplectic area 1 of $S^{2} /(\mathbb{Z} / 2 \mathbb{Z})$; monodromy invariant, the class of $\left(\mu_{\mathrm{h}}\left(\gamma_{1}\right), \mu_{\mathrm{h}}\left(\gamma_{2}\right)\right)=$ $\left\{\left(\begin{array}{ll}1 & 0 \\ 0 & 1\end{array}\right),\left(\begin{array}{ll}0 & 1 \\ 1 & 0\end{array}\right)\right\} \cdot([1 / 2,0],[1 / 2,0])$ under (8.2) . Here $\gamma_{1}, \gamma_{2}$ are loops around the poles of $S^{2}$. Then

$$
M / T=S^{2} /(\mathbb{Z} / 2 \mathbb{Z}), \pi_{1}^{\text {orb }}\left(M / T, p_{0}\right)=\left\langle\gamma_{1} \mid \gamma_{1}^{2}=1\right\rangle \simeq \mathbb{Z} / 2 \mathbb{Z},
$$

and $\mu:\left\langle\gamma_{1} \mid \gamma_{1}^{2}=1\right\rangle \rightarrow T=(\mathbb{R} / \mathbb{Z})^{2}$ is $\mu\left(\gamma_{1}\right)=[1 / 2,0]$. We have a $T$-equivariant symplectomorphism

$$
\left.\widetilde{M / T} \times_{\pi_{1}^{\text {orb }}\left(M / T, p_{0}\right)} T=S^{2} \widetilde{(\mathbb{Z} / 2} \mathbb{Z}\right) \times_{\pi_{1}^{\text {orb }}\left(S^{2} /(\mathbb{Z} / 2 \mathbb{Z}), p_{0}\right)}(\mathbb{R} / \mathbb{Z})^{2} \simeq M .
$$

Remark 8.17. In [42] the authors prove that a compact connected symplectic 4manifold with a symplectic 2-torus action admits an invariant complex structure, and they characterize those which admit a Kähler structure.

\section{FinAL REMARKS}

In this paper we have discussed symplectic Hamiltonian actions as in the works of Ahara, Atiyah, Audin, Delzant, Duistermaat, Hattori, Heckman, Guillemin, Karshon, Kostant, Souriau, and Sternberg [3, 10, 12, 13, 33, 37, 75, 89, 99, 150, among others, and more general symplectic actions as in the works of Benoist, Duistermaat, Frankel, McDuff, Ortega, Ratiu, Tolman, and the author [15, 16, 40, 57, 120, 128, 154. among others. We have described classifications on compact connected manifolds in the following cases.

(1) "Maximal Hamiltonian case": Hamiltonian $T$-action, $\operatorname{dim} M=2 \operatorname{dim} T$.

(2) " $S^{1}$-Hamiltonian case": Hamiltonian $T$-action, $\operatorname{dim} M=4, \operatorname{dim} T=1$.

(3) "Four-dimensional case": $\operatorname{dim} M=4$ and $\operatorname{dim} T=2$.

(4) "Maximal symplectic case": there is a $\operatorname{dim} T$-orbit symplectic orbit.

(5) "Coisotropic case": there is a coisotropic T-orbit.

We have outlined connections of these works with algebraic geometry, in particular Kodaira's classification of complex analytic surfaces [96], toric varieties [41, and log symplectic toric-geometry [69]; geometric topology, in particular the work of Palais and Stewart [124] and Benson and Gordon [17] on torus bundles over tori and nilpotent Lie groups; and integrable systems, in particular the classification of semitoric systems 136, 137.

Some techniques for studying Hamiltonian actions (see for instance the books by Guillemin [70, Guillemin and Sjamaar [74, and Ortega and Ratiu [121]) are useful in the study of general symplectic actions, since many such actions exhibit subgroups which act in a Hamiltonian manner. In the study of Hamiltonian actions one tool that is often used is Morse theory for the components of the momentum map. Any symplectic circle action admits a Morse circle-valued momentum map (see [115, 130]), and there is a well-developed circle-valued Morse theory (see Pajitnov [122 and Farber [50]). Nonetheless, circle-valued Morse theory appears more difficult to apply to general symplectic actions (see [130, Remark 6]). The current understanding of general symplectic actions is quite limited, so we propose the following:

Problem 9.1. Let $G$ be an $m$-dimensional compact connected Lie group. Construct symplectic invariants and classify, up to equivariant symplectomorphisms, 
effective symplectic $G$-actions on compact connected symplectic $2 n$-dimensional manifolds $(M, \omega)$ in terms of these invariants.

We have presented solutions to this problem when $G$ is Abelian and either the $G$-action is Hamiltonian or the orbits are of a certain type (symplectic, Lagrangian, coisotropic); Theorem 8.15 solves it if $G$ is Abelian and $m=n=2$. Solving more cases would be of interest not only in symplectic geometry but also in integrable systems and classical mechanics where the symmetries described by group actions are relevant. Beyond these areas, there have been recent applications to quantum integrable systems [26, 105] which make use of Delzant's theorem (Theorem 6.4) and of the classification of semitoric systems (Theorem 6.18). One may expect other possible applications to spectral geometry if more cases of Problem 9.1 are solved. For instance, the quantization and spectral properties of such actions are interesting problems (a preliminary case is treated in [104]); shedding light on these spectral problems requires understanding the symplectic geometry of their classical counterparts.

Dedication. This paper is dedicated to Professor J. J. Duistermaat (1942-2010). The article [73, edited by V. Guillemin, Á. Pelayo, S. Vũ Ngọc, and A. Weinstein, outlines some of Duistermaat's contributions (see also [138, Section 2.4]). Here is a brief part of the article:

We are honored to pay tribute to Johannes (Hans) J. Duistermaat (1942-2010), a world leading figure in geometric analysis and one of the foremost Dutch mathematicians of the XX century, by presenting a collection of contributions by some of Hans' colleagues, collaborators and students. Duistermaat's first striking contribution was his article 'Fourier integral operators II' with Hörmander (published in Acta Mathematica), a work which he did after his doctoral dissertation. Several influential results in analysis and geometry have the name Duistermaat attached to them, for instance the DuistermaatGuillemin trace formula (1975), Duistermaat's global action-angle theorem (1980), the Duistermaat-Heckman Theorem (1982), and the Duistermaat-Grunbaum bi-spectral theorem (1986). Duistermaat's papers offer an unusual display of originality and technical mastery.

\section{ACKNOWLEDGMENTS}

The author is very thankful to Tudor Ratiu and Alan Weinstein for discussions and for pointing out several useful references, and to an anonymous referee for many helpful comments which have improved the paper.

\section{About THE AUthor}

Álvaro Pelayo is associate professor of mathematics at University of CaliforniaSan Diego. He has been a recipient of the Royal Spanish Mathematical Society Rubio de Francia Prize, the NSF Career Award, and the Senior Lebesgue Chair (Lebesgue Institute). His research spans about 50 publications in symplectic geometry, spectral geometry, and homotopy type theory. 


\section{REFERENCES}

[1] R. Abraham, J. E. Marsden, A. Kelley, and A. N. Kolmogorov, Foundations of mechanics. A mathematical exposition of classical mechanics with an introduction to the qualitative theory of dynamical systems and applications to the three-body problem, With the assistance of Jerrold E. Marsden. Four appendices, one by the author, two by Al Kelley, the fourth, a translation of an article by A. N. Kolmogorov, W. A. Benjamin, Inc., New York-Amsterdam, 1967. MR0220467

[2] R. Abraham and J. E. Marsden, Foundations of mechanics, Benjamin/Cummings Publishing Co., Inc., Advanced Book Program, Reading, Mass., 1978. Second edition, revised and enlarged; With the assistance of Tudor Ratiu and Richard Cushman. MR515141

[3] K. Ahara and A. Hattori, 4-dimensional symplectic $S^{1}$-manifolds admitting moment map, J. Fac. Sci. Univ. Tokyo Sect. IA Math. 38 (1991), no. 2, 251-298. MR1127083

[4] A. Yu. Alekseev, On Poisson actions of compact Lie groups on symplectic manifolds, J. Differential Geom. 45 (1997), no. 2, 241-256. MR1449971

[5] V. Arnold, Sur une propriété topologique des applications globalement canoniques de la mécanique classique (French), C. R. Acad. Sci. Paris 261 (1965), 3719-3722. MR0193645

[6] V. Arnold, Sur la géométrie différentielle des groupes de Lie de dimension infinie et ses applications à l'hydrodynamique des fluides parfaits (French), Ann. Inst. Fourier (Grenoble) 16 (1966), no. fasc. 1, 319-361. MR0202082

[7] V. I. Arnold, Matematicheskie metody klassicheskő mekhaniki. (Russian), Izdat. "Nauka", Moscow, 1974. MR0474390

[8] V. I. Arnold, Fixed points of symplectic diffeomorphisms, Problem XX in Problems of Present Day Mathematics, Mathematical Developments Arising from Hilbert Problems, Proc. Sympos. Pure Math., vol. 28, Amer. Math. Soc, Providence, R. I., 1976, p. 66.

[9] V. I. Arnold, Mathematical methods of classical mechanics, Springer-Verlag, New YorkHeidelberg, 1978. Translated from the Russian by K. Vogtmann and A. Weinstein; Graduate Texts in Mathematics, 60. MR0690288

[10] M. F. Atiyah, Convexity and commuting Hamiltonians, Bull. London Math. Soc. 14 (1982), no. 1, 1-15, DOI 10.1112/blms/14.1.1. MR642416

[11] M. F. Atiyah and R. Bott, The moment map and equivariant cohomology, Topology 23 (1984), no. 1, 1-28, DOI 10.1016/0040-9383(84)90021-1. MR.721448

[12] M. Audin, Hamiltoniens périodiques sur les variétés symplectiques compactes de dimension 4, Géometrie symplectique et mécanique, Proceedings 1988, C. Albert ed., Springer Lecture Notes in Math. 1416 (1990).

[13] M. Audin, The topology of torus actions on symplectic manifolds, Progress in Mathematics, vol. 93, Birkhäuser Verlag, Basel, 1991. Translated from the French by the author. MR.1106194

[14] M. Audin, Vladimir Igorevich Arnold and the invention of symplectic topology, Contact and symplectic topology, Bolyai Soc. Math. Stud., vol. 26, János Bolyai Math. Soc., Budapest, 2014, pp. 1-25, DOI 10.1007/978-3-319-02036-5_1. MR3220939

[15] Y. Benoist, Actions symplectiques de groupes compacts (French, with English summary), Geom. Dedicata 89 (2002), 181-245, DOI 10.1023/A:1014253511289. MR.1890958

[16] Y. Benoist, Correction to "Actions symplectiques de groupes compacts" (French, with English summary), Geom. Dedicata 125 (2007), 251-252.

[17] C. Benson and C. S. Gordon, Kähler and symplectic structures on nilmanifolds, Topology 27 (1988), no. 4, 513-518, DOI 10.1016/0040-9383(88)90029-8. MR976592

[18] N. Berline and M. Vergne, Classes caractéristiques équivariantes. Formule de localisation en cohomologie équivariante (French, with English summary), C. R. Acad. Sci. Paris Sér. I Math. 295 (1982), no. 9, 539-541. MR685019

[19] G. D. Birkhoff, Proof of Poincaré's geometric theorem, Trans. Amer. Math. Soc. 14 (1913), no. 1, 14-22, DOI 10.2307/1988766. MR1500933

[20] P. Birtea, J.-P. Ortega, and T. S. Ratiu, Openness and convexity for momentum maps, Trans. Amer. Math. Soc. 361 (2009), no. 2, 603-630, DOI 10.1090/S0002-9947-08-04689-8. MR2452817

[21] A. V. Bolsinov and A. T. Fomenko, Integrable Hamiltonian systems: Geometry, topology, classification, Chapman \& Hall/CRC, Boca Raton, FL, 2004. Translated from the 1999 Russian original. MR2036760 
[22] A. Cannas da Silva, Lectures in Symplectic Geometry, Springer-Verlag, Berlin (2000).

[23] É. Cartan, La théorie des groupes finis et continus et l'Analysis Situs, Mémorial Sc. Math. XLII (1930) 1-61.

[24] M. Chaperon, Quelques questions de géométrie symplectique, Séminaire Bourbaki, 1982-83, Astérisque 105-106, Soc. Math. Prance, Paris, 1983, pp. 231-249.

[25] M. Chaperon, Une idée du type "géodésiques brisées" pour les systèmes hamiltoniens (French, with English summary), C. R. Acad. Sci. Paris Sér. I Math. 298 (1984), no. 13, 293-296. MR765426

[26] L. Charles, Á. Pelayo, and S. Vũ Ngọc, Isospectrality for quantum toric integrable systems (English, with English and French summaries), Ann. Sci. Éc. Norm. Supér. (4) 46 (2013), no. 5, 815-849. MR 3185353

[27] K. Cieliebak, H. Hofer, J. Latschev, and F. Schlenk, Quantitative symplectic geometry, Dynamics, ergodic theory, and geometry, Math. Sci. Res. Inst. Publ., vol. 54, Cambridge Univ. Press, Cambridge, 2007, pp. 1-44, DOI 10.1017/CBO9780511755187.002. MR2369441

[28] Y. Colin de Verdière and J. Vey, Le lemme de Morse isochore (French), Topology 18 (1979), no. 4, 283-293, DOI 10.1016/0040-9383(79)90019-3. MR.551010

[29] C. C. Conley and E. Zehnder, The Birkhoff-Lewis fixed point theorem and a conjecture of V. I. Arnold, Invent. Math. 73 (1983), no. 1, 33-49, DOI 10.1007/BF01393824. MR707347

[30] S. Curry, Á. Pelayo, X. Tang, Stability of symplectic forms on noncompact manifolds, preprint, February 2017.

[31] V. I. Danilov, The geometry of toric varieties (Russian), Uspekhi Mat. Nauk 33 (1978), no. 2(200), 85-134, 247. MR495499

[32] G. Darboux, Sur le problème de Pfaff, Bulletin des Sciences mathéma. et astrono., 2 série, t. VI; I88z, (1882) 1-46.

[33] T. Delzant, Hamiltoniens périodiques et images convexes de l'application moment (French, with English summary), Bull. Soc. Math. France 116 (1988), no. 3, 315-339. MR984900

[34] J. J. Duistermaat and L. Hörmander, Fourier integral operators. II, Acta Math. 128 (1972), no. 3-4, 183-269, DOI 10.1007/BF02392165. MR0388464

[35] J. J. Duistermaat, Oscillatory integrals, Lagrange immersions and unfolding of singularities, Comm. Pure Appl. Math. 27 (1974), 207-281, DOI 10.1002/cpa.3160270205. MR0405513

[36] J. J. Duistermaat, On global action-angle coordinates, Comm. Pure Appl. Math. 33 (1980), no. 6, 687-706, DOI 10.1002/cpa.3160330602. MR596430

[37] J. J. Duistermaat and G. J. Heckman, On the variation in the cohomology of the symplectic form of the reduced phase space, Invent. Math. 69 (1982), no. 2, 259-268, DOI 10.1007/BF01399506. MR674406

[38] J. J. Duistermaat and G. J. Heckman, Addendum to: "On the variation in the cohomology of the symplectic form of the reduced phase space", Invent. Math. 72 (1983), no. 1, 153-158, DOI 10.1007/BF01389132. MR696693

[39] J. J. Duistermaat and J. A. C. Kolk, Lie groups, Universitext, Springer-Verlag, Berlin, 2000. MR.1738431

[40] J. J. Duistermaat and Á. Pelayo, Symplectic torus actions with coisotropic principal orbits (English, with English and French summaries), Ann. Inst. Fourier (Grenoble) 57 (2007), no. 7, 2239-2327. Festival Yves Colin de Verdière. MR 2394542

[41] J. J. Duistermaat and Á. Pelayo, Reduced phase space and toric variety coordinatizations of Delzant spaces, Math. Proc. Cambridge Philos. Soc. 146 (2009), no. 3, 695-718, DOI 10.1017/S0305004108002077. MR2496353

[42] J. J. Duistermaat and Á. Pelayo, Complex structures on four-manifolds with symplectic two-torus actions, Internat. J. Math. 22 (2011), no. 3, 449-463, DOI 10.1142/S0129167X11006854. MR2782696

[43] J. J. Duistermaat and Á. Pelayo, Topology of symplectic torus actions with symplectic orbits, Rev. Mat. Complut. 24 (2011), no. 1, 59-81, DOI 10.1007/s13163-010-0028-5. MR.2763366

[44] I. Ekeland and H. Hofer, Symplectic topology and Hamiltonian dynamics, Math. Z. 200 (1989), no. 3, 355-378, DOI 10.1007/BF01215653. MR978597

[45] Y. Eliashberg, Rigidity of symplectic and contact structures. Abstracts of reports to Seventh Leningrad International Topology Conference (1982).

[46] Ya. M. Eliashberg, A theorem on the structure of wave fronts and its application in symplectic topology (Russian), Funktsional. Anal. i Prilozhen. 21 (1987), no. 3, 65-72, 96. MR911776 
[47] L. H. Eliasson, Hamiltonian systems with Poisson commuting integrals, PhD thesis, Univ. of Stockholm, 1984.

[48] L. H. Eliasson, Normal forms for Hamiltonian systems with Poisson commuting integralselliptic case, Comment. Math. Helv. 65 (1990), no. 1, 4-35, DOI 10.1007/BF02566590. MR.1036125

[49] H. Flaschka and T. Ratiu, A convexity theorem for Poisson actions of compact Lie groups, Ann. Sci. École Norm. Sup. (4) 29 (1996), no. 6, 787-809. MR1422991

[50] M. Farber, Topology of closed one-forms, Mathematical Surveys and Monographs, vol. 108, American Mathematical Society, Providence, RI, 2004. MR2034601

[51] A. Figalli, J. Palmer, Á. Pelayo, Symplectic G-capacities and integrable systems, arXiv:1511.04499, to appear in Annali della Scuola Normale Superiore di Pisa.

[52] A. Figalli, Á. Pelayo, On the continuity of ball packing density on moduli spaces of toric manifolds, Advances in Geometry 16 (2016) 291-300.

[53] A. Floer, Morse theory for Lagrangian intersections, J. Differential Geom. 28 (1988), no. 3, 513-547. MR965228

[54] A. Floer, Witten's complex and infinite-dimensional Morse theory, J. Differential Geom. 30 (1989), no. 1, 207-221. MR1001276

[55] A. Floer, Symplectic fixed points and holomorphic spheres, Comm. Math. Phys. 120 (1989), no. 4, 575-611. MR 987770

[56] A. Floer, Elliptic methods in variational problems, ICM-90, Mathematical Society of Japan, Tokyo; distributed outside Asia by the American Mathematical Society, Providence, RI, 1990. A plenary address presented at the International Congress of Mathematicians held in Kyoto, August 1990. MR 1126912

[57] T. Frankel, Fixed points and torsion on Kähler manifolds, Ann. of Math. (2) 70 (1959), 1-8, DOI 10.2307/1969889. MR0131883

[58] J. Franks, Generalizations of the Poincaré-Birkhoff theorem, Ann. of Math. (2) 128 (1988), no. 1, 139-151, DOI 10.2307/1971464. MR951509

[59] S. Friedl and S. Vidussi, Twisted Alexander polynomials detect fibered 3-manifolds, Ann. of Math. (2) 173 (2011), no. 3, 1587-1643, DOI 10.4007/annals.2011.173.3.8. MR.2800721

[60] A. Giacobbe, Convexity of multi-valued momentum maps, Geom. Dedicata 111 (2005), 122, DOI 10.1007/s10711-004-1620-y. MR2155173

[61] V. L. Ginzburg, Some remarks on symplectic actions of compact groups, Math. Z. 210 (1992), no. 4, 625-640, DOI 10.1007/BF02571819. MR.1175727

[62] L. Godinho, Semifree symplectic circle actions on 4-orbifolds, Trans. Amer. Math. Soc. 358 (2006), no. 11, 4919-4933, DOI 10.1090/S0002-9947-06-03993-6. MR2231878

[63] L. Godinho, On certain symplectic circle actions, J. Symplectic Geom. 3 (2005), no. 3, 357-383. MR2198781

[64] L. Godinho, Á. Pelayo, and S. Sabatini, Fermat and the number of fixed points of periodic flows, Commun. Number Theory Phys. 9 (2015), no. 4, 643-687, DOI 10.4310/CNTP.2015.v9.n4.a1. MR3506787

[65] L. Godinho and S. Sabatini, New tools for classifying Hamiltonian circle actions with isolated fixed points, Found. Comput. Math. 14 (2014), no. 4, 791-860, DOI 10.1007/s10208014-9204-1. MR3230015

[66] M. Gromov, Pseudo holomorphic curves in symplectic manifolds, Invent. Math. 82 (1985), no. 2, 307-347, DOI 10.1007/BF01388806. MR809718

[67] M. Gromov, Partial differential relations, Ergebnisse der Mathematik und ihrer Grenzgebiete (3) [Results in Mathematics and Related Areas (3)], vol. 9, Springer-Verlag, Berlin, 1986. MR 864505

[68] R. E. Greene and K. Shiohama, Diffeomorphisms and volume-preserving embeddings of noncompact manifolds, Trans. Amer. Math. Soc. 255 (1979), 403-414, DOI 10.2307/1998183. MR542888

[69] M. Gualtieri, S. Li, Á. Pelayo, T. Ratiu, The tropical momentum map: a classification of toric log symplectic manifolds, Math. Ann. (2016). doi:10.1007/s00208-016-1427-9.

[70] V. Guillemin, Moment maps and combinatorial invariants of Hamiltonian $T^{n}$-spaces, Progress in Mathematics, vol. 122, Birkhäuser Boston, Inc., Boston, MA, 1994. MR1301331

[71] V. Guillemin, E. Miranda, A. R. Pires, and G. Scott, Toric actions on b-symplectic manifolds, Int. Math. Res. Not. IMRN 14 (2015), 5818-5848, DOI 10.1093/imrn/rnu108. MR.3384459 
[72] V. Guillemin, E. Miranda, A.R. Pires, G. Scott, Convexity for Hamiltonian torus actions on log symplectic manifolds, arXiv:1412.2488 (to appear in Math. Res. Letters).

[73] Victor Guillemin, Álvaro Pelayo, San Vũ Ngọc and Alan Weinstein, coordinating editors, Remembering Johannes J. Duistermaat (1942-2010), Notices Amer. Math. Soc. 58 (2011), no. 6, 794-802. MR2839924

[74] V. Guillemin and R. Sjamaar, Convexity properties of Hamiltonian group actions, CRM Monograph Series, vol. 26, American Mathematical Society, Providence, RI, 2005. MR.2175783

[75] V. Guillemin and S. Sternberg, Convexity properties of the moment mapping, Invent. Math. 67 (1982), no. 3, 491-513, DOI 10.1007/BF01398933. MR664117

[76] V. Guillemin and S. Sternberg, Symplectic techniques in physics, Cambridge University Press, Cambridge, 1984. MR770935

[77] V. Guillemin and S. Sternberg, Multiplicity-free spaces, J. Differential Geom. 19 (1984), no. 1, 31-56. MR739781

[78] L. Guth, Symplectic embeddings of polydisks, Invent. Math. 172 (2008), no. 3, 477-489, DOI 10.1007/s00222-007-0103-9. MR2393077

[79] H. Hofer, Lagrangian embeddings and critical point theory, Ann. Inst. H. Poincaré Anal. Non Linéaire 2 (1985), no. 6, 407-462. MR831040

[80] H. Hofer, Symplectic capacities, Geometry of low-dimensional manifolds, 2 (Durham, 1989), London Math. Soc. Lecture Note Ser., vol. 151, Cambridge Univ. Press, Cambridge, 1990, pp. 15-34. MR 1171906

[81] H. Hofer, Symplectic invariants, Proceedings of the International Congress of Mathematicians, Vol. I, II (Kyoto, 1990), Math. Soc. Japan, Tokyo, 1991, pp. 521-528. MR1159239

[82] H. Hofer, Arnold and symplectic geometry, Notices Amer. Math. Soc. 59 (2012) 499-502.

[83] H. Hofer and E. Zehnder, Symplectic invariants and Hamiltonian dynamics, Birkhäuser Advanced Texts: Basler Lehrbücher. [Birkhäuser Advanced Texts: Basel Textbooks], Birkhäuser Verlag, Basel, 1994. MR1306732

[84] S. Hohloch, S. Sabatini, and D. Sepe, From compact semi-toric systems to Hamiltonian $S^{1}$-spaces, Discrete Contin. Dyn. Syst. 35 (2015), no. 1, 247-281. MR3286957

[85] D. Jang, Symplectic periodic flows with exactly three equilibrium points, Ergodic Theory Dynam. Systems 34 (2014), no. 6, 1930-1963, DOI 10.1017/etds.2014.56. MR3272779

[86] D. Jang, Symplectic circle actions with isolated fixed points, ProQuest LLC, Ann Arbor, MI, 2015. Thesis (Ph.D.)-University of Illinois at Urbana-Champaign. MR3450412

[87] T. Kajiwara, Tropical toric geometry, Toric topology, Contemp. Math., vol. 460, Amer. Math. Soc., Providence, RI, 2008, pp. 197-207, DOI 10.1090/conm/460/09018. MR2428356

[88] D. M. Kane, J. Palmer, Á. Pelayo, Classifying toric and semitoric fans by lifting equations from $S L_{2}(\mathbb{Z})$, arXiv:1502.07698.

[89] Y. Karshon, Periodic Hamiltonian flows on four-dimensional manifolds, Mem. Amer. Math. Soc. 141 (1999), no. 672, viii+71, DOI 10.1090/memo/0672. MR1612833

[90] Y. Karshon and E. Lerman, Non-compact symplectic toric manifolds, SIGMA Symmetry Integrability Geom. Methods Appl. 11 (2015), Paper 055, 37, DOI 10.3842/SIGMA.2015.055. MR.3371718

[91] Y. Karshon and S. Tolman, Classification of Hamiltonian torus actions with twodimensional quotients, Geom. Topol. 18 (2014), no. 2, 669-716, DOI 10.2140/gt.2014.18.669. MR 3180483

[92] B. Khesin and P. Lee, A nonholonomic Moser theorem and optimal transport, J. Symplectic Geom. 7 (2009), no. 4, 381-414. MR2551999

[93] M. K. Kim, Frankel's theorem in the symplectic category, Trans. Amer. Math. Soc. 358 (2006), no. 10, 4367-4377, DOI 10.1090/S0002-9947-06-03844-X. MR2231381

[94] A. A. Kirillov, Unitary representations of nilpotent Lie groups (Russian), Uspehi Mat. Nauk 17 (1962), no. 4 (106), 57-110. MR0142001

[95] F. Kirwan, Convexity properties of the moment mapping. III, Invent. Math. 77 (1984), no. 3, 547-552, DOI 10.1007/BF01388838. MR759257

[96] K. Kodaira, On the structure of compact complex analytic surfaces. I, Amer. J. Math. 86 (1964), 751-798, DOI 10.2307/2373157. MR0187255

[97] B. Kostant, Orbits, symplectic structures and representation theory, Proc. U.S.-Japan Seminar in Differential Geometry (Kyoto, 1965), Nippon Hyoronsha, Tokyo, 1966, pp. p. 71. MR 0213476 
[98] B. Kostant, Quantization and unitary representations. I. Prequantization, Lectures in modern analysis and applications, III, Springer, Berlin, 1970, pp. 87-208. Lecture Notes in Math., Vol. 170. MR 0294568

[99] B. Kostant, On convexity, the Weyl group and the Iwasawa decomposition, Ann. Sci. École Norm. Sup. (4) 6 (1973), 413-455 (1974). MR0364552

[100] J. L. Koszul, Sur certains groupes de transformations de Lie (French), Géométrie différentielle. Colloques Internationaux du Centre National de la Recherche Scientifique, Strasbourg, 1953, Centre National de la Recherche Scientifique, Paris, 1953, pp. 137-141. MR.0059919

[101] Ç. Kutluhan and C. H. Taubes, Seiberg-Witten Floer homology and symplectic forms on $S^{1} \times M^{3}$, Geom. Topol. 13 (2009), no. 1, 493-525, DOI 10.2140/gt.2009.13.493. MR.2469523

[102] J. L. Lagrange, Mémoire sur la théorie des variations des éléments des planètes, Mémoires de la classe des sciences mathématiques et physiques de l'institut de France, 1808, pp. 1-72.

[103] J. L. Lagrange, Second mémoire sur la théorie de la variation des constantes arbitraires dans les problèmes de mécanique, Mémoires de la classe des sciences mathématiques et physiques de l'institut de France 1809, 343-352.

[104] Y. Le Floch, Á. Pelayo, Symplectic geometry and spectral properties of classical and quantum coupled angular momenta, arXiv:1607.05419.

[105] Y. Le Floch, Á. Pelayo, and S. Vũ Ngọc, Inverse spectral theory for semiclassical JaynesCummings systems, Math. Ann. 364 (2016), no. 3-4, 1393-1413, DOI 10.1007/s00208-0151259-z. MR 3466872

[106] E. Lerman, Symplectic cuts, Math. Res. Lett. 2 (1995), no. 3, 247-258, DOI 10.4310/MRL.1995.v2.n3.a2. MR1338784

[107] E. Lerman and S. Tolman, Hamiltonian torus actions on symplectic orbifolds and toric varieties, Trans. Amer. Math. Soc. 349 (1997), no. 10, 4201-4230, DOI 10.1090/S00029947-97-01821-7. MR1401525

[108] G. W. Mackey, The mathematical foundations of quantum mechanics: A lecture-note volume, W., A. Benjamin, Inc., New York-Amsterdam, 1963. MR0155567

[109] C.-M. Marle, Classification des actions hamiltoniennes au voisinage d'une orbite, C. R. Acad. Sci. Paris Sér. I Math. 299 (1984) 249-252. Modèle d'action hamiltonienne d'un groupe de Lie sur une variété symplectique, Rend. Sem. Mat. Univ. Politec. Torino 43 (1985) 227-251.

[110] J. E. Marsden and T. S. Ratiu, Introduction to mechanics and symmetry, 2nd ed., Texts in Applied Mathematics, vol. 17, Springer-Verlag, New York, 1999. A basic exposition of classical mechanical systems. MR 1723696

[111] J. Marsden and A. Weinstein, Reduction of symplectic manifolds with symmetry, Rep. Mathematical Phys. 5 (1974), no. 1, 121-130. MR0402819

[112] V. P. Maslov, Théorie des perturbations et méthodes asymptotiques, Dunod, GauthierVillars, Paris, 1972. (Translation of 1965 Russian edition.)

[113] K. R. Meyer, Symmetries and integrals in mechanics, Dynamical systems (Proc. Sympos., Univ. Bahia, Salvador, 1971), Academic Press, New York, 1973, pp. 259-272. MR0331427

[114] J. D. McCarthy and J. G. Wolfson, Symplectic gluing along hypersurfaces and resolution of isolated orbifold singularities, Invent. Math. 119 (1995), no. 1, 129-154, DOI 10.1007/BF01245176. MR 1309973

[115] D. McDuff, The moment map for circle actions on symplectic manifolds, J. Geom. Phys. 5 (1988), no. 2, 149-160, DOI 10.1016/0393-0440(88)90001-0. MR.1029424

[116] D. McDuff and D. Salamon, Introduction to symplectic topology, 2nd ed., Oxford Mathematical Monographs, The Clarendon Press, Oxford University Press, New York, 1998. MR.1698616

[117] D. McDuff and S. Tolman, Topological properties of Hamiltonian circle actions, IMRP Int. Math. Res. Pap. (2006), 72826, 1-77. MR2210662

[118] J. Moser, On the volume elements on a manifold, Trans. Amer. Math. Soc. 120 (1965), 286-294, DOI 10.2307/1994022. MR0182927

[119] K. Ono, Some remarks on group actions in symplectic geometry, J. Fac. Sci. Univ. Tokyo Sect. IA Math. 35 (1988), no. 3, 431-437. MR.965009

[120] J.-P. Ortega and T. S. Ratiu, A symplectic slice theorem, Lett. Math. Phys. 59 (2002), no. 1, 81-93, DOI 10.1023/A:1014407427842. MR.1894237 
[121] J.-P. Ortega and T. S. Ratiu, Momentum maps and Hamiltonian reduction, Progress in Mathematics, vol. 222, Birkhäuser Boston, Inc., Boston, MA, 2004. MR2021152

[122] A. V. Pajitnov, Circle-valued Morse theory, De Gruyter Studies in Mathematics, vol. 32, Walter de Gruyter \& Co., Berlin, 2006. MR2319639

[123] J. Palmer, Moduli spaces of semitoric systems, arXiv:1502.07698, to appear in J. of Geom. and Phys.

[124] R. S. Palais and T. E. Stewart, Torus bundles over a torus, Proc. Amer. Math. Soc. 12 (1961), 26-29, DOI 10.2307/2034118. MR0123638

[125] S. Payne, Analytification is the limit of all tropicalizations, Math. Res. Lett. 16 (2009), no. 3, 543-556, DOI 10.4310/MRL.2009.v16.n3.a13. MR2511632

[126] Á. Pelayo, Toric symplectic ball packing, Topology Appl. 153 (2006), no. 18, 3633-3644, DOI 10.1016/j.topol.2006.03.012. MR2270611

[127] Á. Pelayo, Topology of spaces of equivariant symplectic embeddings, Proc. Amer. Math. Soc. 135 (2007), no. 1, 277-288, DOI 10.1090/S0002-9939-06-08310-9. MR2280203

[128] Á. Pelayo, Symplectic actions of 2-tori on 4-manifolds, Mem. Amer. Math. Soc. 204 (2010), no. 959, viii+81, DOI 10.1090/S0065-9266-09-00584-5. MR2640344

[129] Á. Pelayo, A. R. Pires, T. S. Ratiu, and S. Sabatini, Moduli spaces of toric manifolds, Geom. Dedicata 169 (2014), 323-341, DOI 10.1007/s10711-013-9858-x. MR.3175252

[130] Á. Pelayo and T. S. Ratiu, Circle-valued momentum maps for symplectic periodic flows, Enseign. Math. (2) 58 (2012), no. 1-2, 205-219, DOI 10.4171/LEM/58-1-10. MR2985017

[131] Á. Pelayo, T. S. Ratiu, S. Vũ Ngọc, The affine invariant of generalized semitoric systems, arXiv:1307.7516. (Renamed "The affine invariant of nonproper semitoric systems".)

[132] Á. Pelayo, F. Rezakhanlou, Poincaré-Birkhoff theorems in random dynamics, to appear in Trans. Amer. Math. Soc.

[133] Á. Pelayo and B. Schmidt, Maximal ball packings of symplectic-toric manifolds, Int. Math. Res. Not. IMRN 3 (2008), Art. ID rnm139, 24, DOI 10.1093/imrn/rnm139. MR2416999

[134] Á. Pelayo, X. Tang, Moser stability for volume forms on noncompact fiber bundles, arXiv:1607.03800.

[135] Á. Pelayo and S. Tolman, Fixed points of symplectic periodic flows, Ergodic Theory Dynam. Systems 31 (2011), no. 4, 1237-1247, DOI 10.1017/S0143385710000295. MR2818694

[136] Á. Pelayo and S. Vũ Ngọc, Semitoric integrable systems on symplectic 4-manifolds, Invent. Math. 177 (2009), no. 3, 571-597, DOI 10.1007/s00222-009-0190-x. MR2534101

[137] Á. Pelayo and S. Vũ Ngọc, Constructing integrable systems of semitoric type, Acta Math. 206 (2011), no. 1, 93-125, DOI 10.1007/s11511-011-0060-4. MR2784664

[138] Á. Pelayo and S. Vũ Ngọc, Symplectic theory of completely integrable Hamiltonian systems, Bull. Amer. Math. Soc. (N.S.) 48 (2011), no. 3, 409-455, DOI 10.1090/S0273-0979-201101338-6. MR 2801777

[139] Á. Pelayo and S. Vũ Ngọc, Hofer's question on intermediate symplectic capacities, Proc. Lond. Math. Soc. (3) 110 (2015), no. 4, 787-804, DOI 10.1112/plms/pdu066. MR3335287

[140] Á. Pelayo, V. Voevodsky, M. A. Warren, A univalent formalization of the p-adic numbers, Mathematical Structures in Computer Science, 25 (2014), 1147-1171.

[141] H. Poincaré, Les Méthodes Nouvelles de la Mécanique Céleste, Tome I, Paris, GauthierViltars, 1892. Republished by Blanchard, Paris, 1987.

[142] H. Poincaré, Sur un théorème de géométrie, Rend. Circ. Mat. Palermo 33 (1912) 375-407.

[143] L. Polterovich, The geometry of the group of symplectic diffeomorphisms, Lectures in Mathematics ETH Zürich, Birkhäuser Verlag, Basel, 2001. MR.1826128

[144] R. C. Robinson, Generic properties of conservative systems, Amer. J. Math. 92 (1970), 562-603, DOI 10.2307/2373361. MR0273640

[145] R. C. Robinson, Generic one parameter families of symplectic matrices, Amer. J. Math. 93 (1971), 116-122, DOI 10.2307/2373451. MR0377995

[146] H. Rüssmann, Über das Verhalten analytischer Hamiltonscher Differentialgleichungen in der Nähe einer Gleichgewichtslösung (German), Math. Ann. 154 (1964), 285-300, DOI 10.1007/BF01362565. MR0179409

[147] I. E. Segal, Quantization of nonlinear systems, J. Mathematical Phys. 1 (1960), 468-488, DOI 10.1063/1.1703683. MR0135093

[148] R. Sjamaar and E. Lerman, Stratified symplectic spaces and reduction, Ann. of Math. (2) 134 (1991), no. 2, 375-422, DOI 10.2307/2944350. MR.1127479 
[149] J.-M. Souriau, Quantification géométrique (French, with English summary), Comm. Math. Phys. 1 (1966), 374-398. MR0207332

[150] J. P. Souriau, Structure des Systèmes Dynamiques. Dunoud, Paris 1970. English translation by R.H. Cushman and G.M. Tuynman. Progress in Mathematics, 149. Birkhäuser Boston, 1997.

[151] S. Sternberg: Celestial Mechanics. II, W. A. Benjamin, New York, 1969.

[152] W. P. Thurston, Some simple examples of symplectic manifolds, Proc. Amer. Math. Soc. 55 (1976), no. 2, 467-468, DOI 10.2307/2041749. MR.0402764

[153] W. Thurston, The Geometry and Topology of 3-manifolds, Electronic version 1.1, March 2002, http://www.msri.org/publications/books/gt3m/.

[154] S. Tolman, Non-Hamiltonian actions with isolated fixed points, arXiv:1510.02829.

[155] S. Tolman and J. Weitsman, On semifree symplectic circle actions with isolated fixed points, Topology 39 (2000), no. 2, 299-309, DOI 10.1016/S0040-9383(99)00011-7. MR.1722020

[156] J. Vey, Sur certains systèmes dynamiques séparables (French), Amer. J. Math. 100 (1978), no. 3, 591-614, DOI 10.2307/2373841. MR0501141

[157] C. Wacheux, Asymptotics of action variables near semi-toric singularities, J. Geom. Phys. 98 (2015), 28-39, DOI 10.1016/j.geomphys.2015.07.023. MR.3414940

[158] A. Weinstein, Symplectic manifolds and their Lagrangian submanifolds, Advances in Math. 6 (1971), 329-346 (1971), DOI 10.1016/0001-8708(71)90020-X. MR0286137

[159] A. Weinstein, Lectures on symplectic manifolds, American Mathematical Society, Providence, R.I., 1977. Expository lectures from the CBMS Regional Conference held at the University of North Carolina, March 8-12, 1976; Regional Conference Series in Mathematics, No. 29. MR0464312

[160] A. Weinstein, Symplectic geometry, Bull. Amer. Math. Soc. (N.S.) 5 (1981), no. 1, 1-13, DOI 10.1090/S0273-0979-1981-14911-9. MR614310

[161] A. Weinstein, The local structure of Poisson manifolds, J. Differential Geom. 18 (1983), no. 3, 523-557. MR723816

[162] A. Weinstein, On extending the Conley-Zehnder fixed point theorem to other manifolds, Nonlinear functional analysis and its applications, Part 2 (Berkeley, Calif., 1983), Proc. Sympos. Pure Math., vol. 45, Amer. Math. Soc., Providence, RI, 1986, pp. 541-544. MR843640

[163] A. Weinstein, Poisson geometry of discrete series orbits, and momentum convexity for noncompact group actions, Lett. Math. Phys. 56 (2001), no. 1, 17-30, DOI 10.1023/A:1010913023218. EuroConférence Moshé Flato 2000, Part I (Dijon). MR 1848163

[164] H. Weyl, The classical groups, Princeton Landmarks in Mathematics, Princeton University Press, Princeton, NJ, 1997. Their invariants and representations; Fifteenth printing; Princeton Paperbacks. MR 1488158

[165] C. Woodward, The classification of transversal multiplicity-free group actions, Ann. Global Anal. Geom. 14 (1996), no. 1, 3-42, DOI 10.1007/BF00128193. MR.1375064

[166] S. T. Yau, Remarks on the group of isometries of a Riemannian manifold, Topology 16 (1977), no. 3, 239-247, DOI 10.1016/0040-9383(77)90004-0. MR.0448379

[167] E. Zehnder, The Arnold conjecture for fixed points of symplectic mappings and periodic solutions of Hamiltonian systems, Proceedings of the International Congress of Mathematicians, Vol. 1, 2 (Berkeley, Calif., 1986), Amer. Math. Soc., Providence, RI, 1987, pp. 1237-1246. $\operatorname{MR} 934328$

Department of Mathematics, University of California, San Diego, 9500 Gilman Drive \#0112, La Jolla, CAlifornia 92093-0112

E-mail address: alpelayo@math.ucsd.edu 